RUHR UNIVERSITÄT BOCHUM

\title{
Massively Parallel Multiphase Field Simulations
}

\author{
Dissertation zur \\ Erlangung des Grades \\ Doktor-Ingenieur \\ der \\ Fakultät für Maschinenbau \\ der Ruhr-Universität Bochum \\ Von \\ Marvin Tegeler \\ aus Gelsenkirchen \\ Bochum, 2017
}


Dissertation eingereicht am: 16.10.2017

Tag der mündlichen Prüfung: 7.12.2017

Erstgutachter: Prof. Dr. Ingo Steinbach

Zweitgutachter: Prof. Dr. Godehard Sutmann 



\section{Abstract}

The multiphase field method is a commonly used tool to simulate the evolution of microstructure during materials processing. Even when using a concept called active parameter tracking, which substantially reduces the computational complexity, the resource demands of the multiphase field method remain significant in both time and memory.

A hybrid-parallelization is presented, that combines MPI and OpenMP and allows considerably larger system sizes. Due to the utilization of active parameter tracking the computational load can be heterogeneously distributed in the system, which makes load-balancing necessary in order to obtain an efficient parallelization. In this thesis two different load-balancing schemes are presented. The first uses graphpartitioning and an adaptive sub-domain decomposition. The second is based on the multiphase field method itself.

Results are presented for performance benchmarks as well as for a variety of applications, including grain growth in polycrystalline materials with hundreds of thousands of different phase fields and $\mathrm{Mg}-\mathrm{Al}$ alloy solidification as well as a coupling with the Lattice-Boltzmann method. One of the load-balancing schemes is also applied to a cell-based particle method. In addition a way of utilizing the multiphase field method is shown that allows the construction of microstructures and the corresponding Laguerre generator points with a given grain size distribution. 


\section{Declaration/Erklärung}

I, Marvin Tegeler, born on July, 31st, 1985 in Gelsenkirchen, hereby declare that this dissertation is my own work.

With explicit permission of my advisors Prof. Dr. Ingo Steinbach and Prof. Dr. Godehard Sutmann, parts of this work were already published in scientific journals:

- M. Tegeler, A. Monas, and G. Sutmann, "Massively parallel multiphase field simulations," in Proceedings of Fourth International Conference on Parallel, Distributed, Grid and Cloud Computing for Engineering, 2015

- M. Tegeler, A. Monas, O. Shchyglo, I. Steinbach, and F. Varnik, "Effect of microstructure during dendritic solidification on melt flow: A phase-field-latticeboltzmann study," in Proceedings of the 5th Decennial International Conference on Solidification Processing, 2017

- M. Tegeler, O. Shchyglo, R. D. Kamachali, A. Monas, I. Steinbach, and G. Sutmann, "Parallel multiphase field simulations with OpenPhase," Computer Physics Communications, vol. 215, pp. -, 2017 
Hiermit erkläre ich, Marvin Tegeler, geboren am 31 Juli 1985 in Gelsenkirchen, die vorliegende Dissertation eigenhändig angefertigt zu haben.

Im Einvernehmen und mit der ausdrücklichen Genehmigung meiner Betreuer, Prof. Dr. Ingo Steinbach und Prof. Dr. Godehard Sutmann, wurden Teile der Arbeit bereits in Fachzeitschriften veröffentlicht:

- M. Tegeler, A. Monas, and G. Sutmann, "Massively parallel multiphase field simulations," in Proceedings of Fourth International Conference on Parallel, Distributed, Grid and Cloud Computing for Engineering, 2015

- M. Tegeler, A. Monas, O. Shchyglo, I. Steinbach, and F. Varnik, "Effect of microstructure during dendritic solidification on melt flow: A phase-field-latticeboltzmann study," in Proceedings of the 5th Decennial International Conference on Solidification Processing, 2017

- M. Tegeler, O. Shchyglo, R. D. Kamachali, A. Monas, I. Steinbach, and G. Sutmann, "Parallel multiphase field simulations with OpenPhase," Computer Physics Communications, vol. 215, pp. -, 2017

(Promotionsordnung §11, [4]). 


\section{Acknowledgements}

Firstly, I would like to express my sincere gratitude to my advisors Prof. Dr. Ingo Steinbach and Prof. Dr. Godehard Sutmann for the continuous support of my doctoral studies and related research, for their patience, motivation, knowledge, flexibility and empathy, for the fruitful discussions and the resulting new and interesting views.

My sincere thanks also go to my current and former co-workers at the Interdisciplinary Centre for Advanced Materials Simulations (ICAMS) and especially to Dr. Oleg Shchyglo, apl. Prof. Dr. Fathollah Varnik, Dr. Reza Darvishi Kamachali, Dr. Efim Borukhovich, Dr. Philipp Engels and Dr. Alexander Monas for the interesting scientific discussions and the collaborations in the development of OpenPhase. Secondly I want to thank Dr. Christoph Begau and Dr. Carlos Teijeiro Barjas of high-performance computing group at ICAMS for intense and fruitful discussions on programming and parallelization topics.

Special thanks go to my mother and my late father, who supported me throughout my life and my studies.

This work was financially supported by ThyssenKrupp Steel Europe AG. The author gratefully acknowledge the computing time granted on the supercomputer JURECA at Jülich Supercomputing Centre (JSC). 



\section{Contents}

1 Introduction 13

2 The Multiphase Field Method $\quad \mathbf{1 5}$

2.1 The Multiphase Field Method in OpenPhase . . . . . . . . . . . . . . 15

2.2 Driving-forces . . . . . . . . . . . . . . . . . 16

2.3 Discretization . . . . . . . . . . . . . . . . . . . . . . . . 19

2.4 Driving-force Averaging . . . . . . . . . . . . . . . . . . . . 20

2.5 Sparse Storages . . . . . . . . . . . . . . . . . . . . . . 21

2.6 Time Stepping . . . . . . . . . . . . . . . . . . 22

3 The Lattice-Boltzmann Method 23

3.1 Decaying Taylor-Vortex . . . . . . . . . . . . . . . . . 25

3.2 The Exact Difference Method . . . . . . . . . . . . . . . . . . 26

3.3 Fluid-Solid-Interaction . . . . . . . . . . . . . . . . 27

4 High-Performance Computing 29

4.1 Parallel Programming Models . . . . . . . . . . . . . . . . . . . . . . 29

4.1.1 Distributed-Memory Parallelism . . . . . . . . . . . . . . . 30

4.1.2 Shared-Memory Parallelism . . . . . . . . . . . . . . . 30

4.1.3 Hybrid-Parallelism . . . . . . . . . . . . . . . . . . 31

4.2 Estimating Speedup . . . . . . . . . . . . . . . . . . 31

4.3 Load-balancing . . . . . . . . . . . . . . . . . . . . 33

4.4 Other Parallel Phase Field Implementations . . . . . . . . . . . . . . 35

5 Parallelization $\quad 37$

5.1 Domain-Decomposition . . . . . . . . . . . . . . . 38

5.1 .1 Wide Halo . . . . . . . . . . . . . . . . . . . . . . . . . . 39

5.1.2 Communication Pattern . . . . . . . . . . . . . 40 
5.2 Load-balancing . . . . . . . . . . . . . . . . . . 43

5.2 .1 Graph-partitioning . . . . . . . . . . . . . . . 44

5.2 .2 Bisection . . . . . . . . . . . . . . . . . . 47

5.2 .3 Estimation of Computational Load . . . . . . . . . . . . 50

5.2.4 Determination of the Cutting Plane . . . . . . . . . . . . . 51

5.2 .5 Data Migration . . . . . . . . . . . . . . . . 53

5.2 .6 The Full Balancing Algorithm . . . . . . . . . . . . . 54

5.2 .7 Dynamic Load-balancing . . . . . . . . . . . . . . . . . 54

5.3 Parallel I/O . . . . . . . . . . . . . . . . . . . . 56

6 Applications $\quad 59$

6.1 Normal Grain Growth . . . . . . . . . . . . . . . . . . . 59

6.1 .1 Results and Discussion . . . . . . . . . . . . . . . 61

6.2 Scaling Benchmark for Grain Growth Simulations . . . . . . . . . . 64

6.2.1 Uniform Distribution of Particles . . . . . . . . . . . . . 66

6.2 .2 Gaussian Distribution of Particles . . . . . . . . . . . . 67

6.2 .3 Moving Particles . . . . . . . . . . . . . . . . . . . . . . 69

$6.3 \mathrm{Mg}$-Al Alloy Solidification . . . . . . . . . . . . . . . . . 71

6.4 Chemical Driving-forces and Diffusion . . . . . . . . . . . 75

6.5 Scaling Benchmark for Mg-Al Alloy Solidification . . . . . . . . 76

6.5.1 Uniform Distribution of Particles . . . . . . . . . . . . 76

6.5.2 Gaussian Distribution of Particles . . . . . . . . . . . . 77

6.5.3 Uniform Particle Distribution in one Quadrant of the Domain 80

6.6 Effect of Solidification on the Fluid Flow . . . . . . . . . . . . . 81

6.6 .1 Simulation Setup . . . . . . . . . . . . . . . . . 81

6.6 .2 Darcy's Law . . . . . . . . . . . . . . . . . . . . . . . 82

6.6 .3 Carman-Kozeny Equation . . . . . . . . . . . . . . . 86

7 Transfer of the Multiphase Field Method $\quad 87$

7.1 RVE-Generation with the Multiphase Field Method . . . . . . . . 87

7.1.1 Microstructure Generation Using Multiphase Field . . . . . 88

7.1 .2 Inverse Laguerre Tessellation . . . . . . . . . . . . . . . . 89

7.1.3 Low Contrast Examples . . . . . . . . . . . . . . . . . 91

7.1.4 High Contrast Examples . . . . . . . . . . . . . . . . . 95

7.1 .5 Conclusions . . . . . . . . . . . . . . . . . 95 
7.2 Load-balancing with the Multiphase Field Method . . . . . . . . . . . 99

7.2.1 Implementation of the Multiphase-Load-balancer . . . . . . . 99

7.2.2 Load-balancing Multiphase Field Simulations . . . . . . . . . 100

7.2.3 Load-balancing MD-Simulations . . . . . . . . . . . . . . . 106

7.2.4 Example: Copper Cube . . . . . . . . . . . . . . 109

7.2.5 Example: Multiple Cubes . . . . . . . . . . . . . . . . 112

8 Summary and Conclusions $\quad 115$

9 Appendix 117

9.1 Parallel OpenPhase Tutorial . . . . . . . . . . . . . . . . . . 117

9.1 .1 Installation . . . . . . . . . . . . . . . . . 117

9.1 .2 Getting Started . . . . . . . . . . . . . 117

$\begin{array}{ll}\text { Bibliography } & 123\end{array}$ 



\section{Introduction}

For simulations of the evolution of micro-structure during the processing of materials the multi phase-field method is widely used $[5,6,7,7]$. It is used for processes such as precipitation [8, 9], particle pinning [10], grain growth [11, 12], nanograin growth [13], solidification [14, 15] and recrystallization [16]. This method describes the dynamic of the interface between zones with different properties that are described by auxiliary functions called phase-fields. These functions form a partition of unity and provide a finite diffuse interface between grains. As simulations of statistical relevance can require thousand of different phase fields and large system sizes, the requirements of the multiphase method on the computational resources can be significant, both on memory and time.

However, time is a precious resource that should be used as efficiently as possible. This is true for life itself as well as in science. Researchers seek results as soon as possible, therefore their simulation tools need to be as efficient as possible. Especially in parallelized simulation tools that consist of many individual threads and processes unnecessary waiting times should be avoided in order to keep the wall times of simulations as short as possible. The goal is to provide a robust parallel framework that offers a high performance and excellent scaling behavior for a wide variety of applications, such as normal grain growth and alloy solidification.

In this thesis a framework for a load-balanced parallel simulation tool for the simulation of micro-structure evolution is presented.

The parallelization framework has been implemented in OpenPhase [17], an opensource library that implements the multiphase field method.

The first part of this work gives an introduction to the multiphase field method and presents its implementation in OpenPhase. OpenPhase uses sparse storages and 
active parameter tracking in order to reduce the memory requirements and computation time. This results in an heterogeneous distribution of the workload, which requires load-balancing techniques to avoid unnecessary waiting times in parallel applications.

A distributed-memory parallelization is needed as large scale simulations on large computational grids with thousands of different phase fields exceed the capabilities of single node computer architectures. Multiple attempts have been conducted to parallelize phase field simulations in order to treat large systems $[18,19,20]$. However, both [18] and [20] are restricted to a limited number of phase field and do not implement the multiphase field method. As [19] points out the parallel efficiency and the load-balancing capabilities of the multiphase field software PACE3D [21] is limited by the lack of a three-dimensional domain decomposition. The well-known

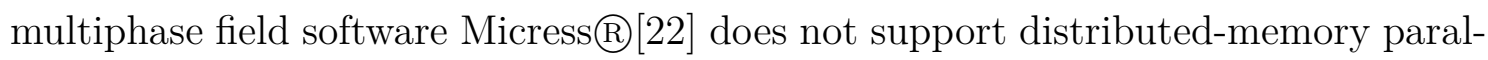
lelization.

The presented thesis expands the articles $[1,3]$ that present the hybrid parallelization of OpenPhase and the article [2] on the coupling with the Lattice-Boltzmann method.

Furthermore this thesis will present two ways to transfer the multiphase field method to other problems by using appropriate driving forces. One is the construction of Laguerre generator points that create tessellation with a given cell size distribution. The multiphase field method is used as a way to create an intermediate microstructure, which is then approximated by an inverse Laguerre tessellation.

The other is the utilization of the multiphase field method as a load-balancing scheme. As a good load-balancing scheme not only balances the workload but also reduces communication as much as possible, the multiphase field seems like a good fit, as it naturally reduces the interface between processes. This load-balancer is applied to the phase field method itself as well as a cell-based particle method. 


\section{The Multiphase Field Method}

The phase field model has a wide variety of applications for the simulation of materials processing $([6],[23])$ as well as image processing $([24,25])$. The model allows the description of interfaces between sub-domains $\Omega_{i} \subset \Omega$ in a domain $\Omega \subset \mathbb{R}^{d}$. In the following these sub-domains will be referred to as phases and should not be confused with sub-domains in the context of domain decomposition methods. The phase-fields $\phi_{\alpha}: \Omega \times \mathbb{R} \rightarrow[0,1]$ are auxiliary functions that distinguish between different phases and describe their evolution. A function value of 1 indicates the bulk of the phase and a value of 0 indicates the absence of this phase. Phases are connected by a diffuse interface that is described a smooth transition of the phase field functions $\phi_{\alpha}$. The phase-fields $\phi_{\alpha}$ fulfill the condition

$$
\sum_{\alpha=1}^{\infty} \phi_{\alpha}=1
$$

in every point $x \in \Omega$ of the domain $\Omega$. In the following the multiphase field model implemented in OpenPhase will be presented in detail.

\subsection{The Multiphase Field Method in OpenPhase}

As presented in [3] the evolution of phase fields $\phi_{\alpha}$ in OpenPhase is determined by

$$
\dot{\phi}_{\alpha}=\frac{\partial}{\partial t} \phi_{\alpha}(x, t)=-\sum_{\beta=1}^{N} \frac{\pi^{2} \mu_{\alpha \beta}}{8 \eta N}\left[\frac{\delta F}{\delta \phi_{\alpha}}-\frac{\delta F}{\delta \phi_{\beta}}\right],
$$

with the interface mobility $\mu_{\alpha \beta}$ between phase fields $\phi_{\alpha}$ and $\phi_{\beta}$ and a free energy

$$
F=\int_{\Omega}\left\{f^{\mathrm{GB}}+\ldots\right\} d V
$$


and the number of phase fields that make a contribution

$$
N:=N(x):=\sum_{\alpha=1}^{\infty} \chi_{T(x)}\left(\phi_{\alpha}\right)
$$

with

$$
T(x):=\left\{\phi \in C^{2}(\Omega) \mid \phi(x) \neq 0 \vee \nabla \phi(x) \neq 0 \vee \nabla^{2} \phi(x) \neq 0\right\}
$$

The interfacial free energy density is determined by

$$
f^{\mathrm{GB}}=\sum_{\alpha \neq \beta} \frac{4 \sigma_{\alpha \beta}}{\eta}\left[-\frac{\eta^{2}}{\pi^{2}} \nabla \phi_{\alpha} \cdot \nabla \phi_{\beta}+\phi_{\alpha} \phi_{\beta}\right] .
$$

with the interface width $\eta$ and the energy $\sigma_{\alpha \beta}$.

Following [23] and [6] with $I_{\alpha}:=\nabla^{2} \phi_{\alpha}+\frac{\pi^{2}}{\eta^{2}} \phi_{\alpha}$ we get

$$
\begin{aligned}
\dot{\phi}_{\alpha}^{\mathrm{GB}} & =\sum_{\substack{\beta=1 \\
\beta \neq \alpha}}^{N} \frac{\mu_{\alpha \beta}}{N}\left[\sum_{\substack{\gamma=1 \\
\gamma \neq \beta}}^{N}\left(\sigma_{\beta \gamma}-\sigma_{\alpha \gamma}\right) I_{\gamma}\right] \\
& =\frac{1}{N} \sum_{\substack{\beta=1 \\
\beta \neq \alpha}}^{N} \mu_{\alpha \beta}\left[\sigma_{\alpha \beta}\left[I_{\alpha}-I_{\beta}\right]+\sum_{\substack{\gamma=1 \\
\alpha \neq \gamma \neq \beta}}^{N}\left(\sigma_{\beta \gamma}-\sigma_{\alpha \gamma}\right) I_{\gamma}\right] \\
& =\frac{1}{N} \sum_{\substack{\beta=1 \\
\beta \neq \alpha}}^{N} \dot{\psi}_{\alpha \beta}^{\mathrm{GB}}
\end{aligned}
$$

with the interface field $\psi_{\alpha \beta}^{\mathrm{GB}}$ for the contribution of the grain boundary.

\subsection{Driving-forces}

In equation 2.3 additional contributions $f^{\mathrm{AD}}$ to the energy density can be considered by expanding the free energy to

$$
F=\int_{\Omega}\left\{f^{\mathrm{GB}}+f^{\mathrm{AD}}\right\} d V
$$


Using the variational derivation as above we obtain

$$
\dot{\phi}_{\alpha}=\sum_{\substack{\beta=1 \\ \beta \neq \alpha}}^{N} \frac{\mu_{\alpha \beta}}{N}\left[\sum_{\substack{\gamma=1 \\ \gamma \neq \beta}}^{N}\left(\sigma_{\beta \gamma}-\sigma_{\alpha \gamma}\right) I_{\gamma}+\frac{\pi^{2}}{8 \eta} \Delta g_{\alpha \beta}\right],
$$

with a driving force

$$
\Delta g_{\alpha \beta}=\frac{\partial f^{\mathrm{AD}}}{\partial \phi_{\beta}}-\frac{\partial f^{\mathrm{AD}}}{\partial \phi_{\alpha}} .
$$

The main advantage of the phase field model used in OpenPhase is the rigorous consideration of the interface properties. One of the key points of the model is the assumption that a steady phase field contour along the interface normal is maintained at all times during the simulation which also ensures constant width of the interface. Such a steady phase field contour, also called traveling wave solution, is important for the correct consideration of the interface properties and the resulting interface kinetics.

Depending on the origin of the driving force $\Delta g$ and context in which the phase field method is utilized, the driving force $\Delta g$ might vary across the interface. This can significantly alter the phase field profile across the interface which affects interface kinetics. However, it is important to note, that the correct phase field profile across the interface can only be obtained if the driving force $\Delta g$ is constant across the interface and varies only along the interface. Therefore, we replace $\Delta g_{\alpha \beta}$ in equation 6.9 with $h^{\prime} \Delta \bar{g}_{\alpha \beta}$ with an average driving force $\Delta \bar{g}_{\alpha \beta}$, that is constant along the normal direction of the interface. In order to guaranty that the driving force contribution to the phase field evolution is independent of the chosen space discretization (the same is true for the interface curvature contribution), the prefactor $h^{\prime}=\frac{8}{\pi} \sqrt{\phi_{\alpha} \phi_{\beta}}$ in front of the driving force $\Delta \bar{g}_{\alpha \beta}$ is obtained by integrating the traveling wave profile 
(for more details see [6]). We finally get

$$
\begin{aligned}
\dot{\phi}_{\alpha} & =\sum_{\substack{\beta=1 \\
\beta \neq \alpha}}^{N} \frac{\mu_{\alpha \beta}}{N}\left[\sum_{\substack{\gamma=1 \\
\gamma \neq \beta}}^{N}\left(\sigma_{\beta \gamma}-\sigma_{\alpha \gamma}\right) I_{\gamma}+\frac{\pi}{\eta} \sqrt{\phi_{\alpha} \phi_{\beta}} \Delta \bar{g}_{\alpha \beta}\right], \\
& =\frac{1}{N} \sum_{\substack{\beta=1 \\
\beta \neq \alpha}}^{N} \dot{\psi}_{\alpha \beta}^{\mathrm{GB}}+\frac{1}{N} \sum_{\substack{\beta=1 \\
\beta \neq \alpha}}^{N} \mu_{\alpha \beta} \frac{\pi}{\eta} \sqrt{\phi_{\alpha} \phi_{\beta}} \Delta \bar{g}_{\alpha \beta}, \\
& =\frac{1}{N} \sum_{\substack{\beta=1 \\
\beta \neq \alpha}}^{N} \dot{\psi}_{\alpha \beta}^{\mathrm{GB}}+\frac{1}{N} \sum_{\substack{\beta=1 \\
\beta \neq \alpha}}^{N} \dot{\psi}_{\alpha \beta}^{\mathrm{AD}}, \\
& =\dot{\phi}_{\alpha}^{\mathrm{GB}}+\dot{\phi}_{\alpha}^{\mathrm{AD}} .
\end{aligned}
$$

Considering equation 2.13 we notice that for a naive implementation the computational effort would scale with $O\left(n^{3}\right)$, where $n$ is the number of phase fields. This would create an unsuitable amount of effort in simulation with a high number of different phase fields. Vedantam [26] introduced a technique known as active parameter tracking, that reduced the computational effort significantly by discarding contributions of phase fields that are determined to be insignificant. In [26] the double well potential is used for which the profile of the diffuse interface is determined by a tanh-function, so that phase field values are close to, but not really 0 or 1 . Therefore in this case active parameter tracking affects the results of the simulation as phase field values close to 0 or 1 are cut off. Using the double obstacle potential, which has a sin -profile, we notice:

Remark 2.2.1. If both $\phi_{k}(x)=0$ and $\nabla^{2} \phi_{k}(x)=0$ then phase $k$ does not make a contribution to $\dot{\psi}_{i j}^{\mathrm{GB}}$.

Therefore a simulation using the double obstacle potential can safely skip the computation of contributions by phase fields $\phi$ with $\phi_{k}(x)=0$ and $\nabla^{2} \phi_{k}(x)=0$.

Remark 2.2.2. $\dot{\phi}_{i}^{n}(x)>0$ is possible even if both $\phi_{i}^{n}(x)=0$ and $\nabla^{2} \phi_{i}^{n}(x)=0$. This means a nucleation event is triggered that would create a new phase. By processing 
these nucleation events separately, we can modify equation 2.8 to

$$
\begin{aligned}
\dot{\phi}_{\alpha}=\chi_{\alpha} \frac{1}{N} & {\left[\sum_{\substack{\beta=1 \\
\beta \neq \alpha}}^{N} \mu_{\alpha \beta}\left[\sigma_{\alpha \beta}\left[I_{\alpha}-I_{\beta}\right]+\sum_{\substack{\gamma=1 \\
\alpha \neq \gamma \neq \beta}}^{N}\left(\sigma_{\beta \gamma}-\sigma_{\alpha \gamma}\right) I_{\gamma}\right]\right.} \\
& \left.+\sum_{\substack{\beta=1 \\
\beta \neq \alpha}}^{N} \mu_{\alpha \beta} \frac{\pi}{\eta} \sqrt{\phi_{\alpha} \phi_{\beta}} \Delta \bar{g}_{\alpha \beta}\right],
\end{aligned}
$$

by using the indicator function

$$
\chi_{i}:= \begin{cases}1 & \text { if } \phi_{i} \neq 0 \vee \nabla^{2} \phi_{i} \neq 0 \\ 0 & \text { else }\end{cases}
$$

which allows us to skip certain computations. In example 6.3 an explicit nucleation model is utilized.

\subsection{Discretization}

In OpenPhase the domain $\Omega \subset \mathbb{R}^{3}$ is discretized by a Cartesian grid $\mid$ the set $\Omega_{h} \subset \Omega$ contains the grid points $x_{h}$ in $\Omega$ with a grid spacing $h$. The phase fields are represented on grid functions $\phi_{\alpha}^{h}$. The evolution of these grid functions over time is discretized by an explicit first order scheme

$$
\begin{aligned}
\phi_{\alpha}^{h}\left(x_{h}, t_{n}+\Delta t_{n}\right) & =\phi_{\alpha}^{h}\left(x_{h}, t_{n}\right)+\Delta t_{n} \dot{\phi}_{\alpha}^{\mathrm{GB}, h}\left(x_{h}, t_{n}\right) \\
& +\Delta t_{n} \dot{\phi}_{\alpha}^{\mathrm{CH}, h}\left(x_{h}, t_{n}\right) \forall x_{h} \in \Omega_{h}
\end{aligned}
$$

with the separate updates $\dot{\phi}_{\alpha}^{\mathrm{GB}, h}\left(x_{h}, t_{n}\right)$ and $\dot{\phi}_{\alpha}^{\mathrm{CH}, h}\left(x_{h}, t_{n}\right)$ in each grid point $x_{h} \in \Omega_{h}$ at time $t_{n}$ given by

$$
\dot{\phi}_{\alpha}^{\mathrm{GB}, h}\left(x_{h}, t_{n}\right)=\chi_{\alpha}\left(x_{h}, t_{n}\right) \frac{1}{N} \sum_{\substack{\beta=1 \\ \beta \neq \alpha}}^{N} \dot{\psi}_{\alpha \beta}^{\mathrm{GB}}\left(x_{h}, t_{n}\right),
$$


and

$$
\begin{aligned}
& \dot{\phi}_{\alpha}^{\mathrm{CH}, h}\left(x_{h}, t_{n}\right)= \\
& \chi_{\alpha}\left(x_{h}, t_{n}\right) \frac{1}{N} \sum_{\substack{\beta=1 \\
\beta \neq \alpha}}^{N} \mu_{\alpha \beta}\left(x_{h}, t_{n}\right) \frac{\pi}{\eta} \sqrt{\phi_{\alpha}\left(x_{h}, t_{n}\right) \phi_{\beta}\left(x_{h}, t_{n}\right)} \Delta \bar{g}_{\alpha \beta}\left(x_{h}, t_{n}\right) .
\end{aligned}
$$

The Laplacians $\nabla^{2} \phi_{i}$ in $I_{\alpha}$ are approximated by the compact $3 \times 3 \times 3$ finite difference stencil $\nabla_{h}^{2} \phi^{h}$ to get the updates

$$
\dot{\psi}_{\alpha \beta}^{\mathrm{GB}}\left(x_{h}, t_{n}\right)=\mu_{\alpha \beta}\left[\sigma_{\alpha \beta}\left[I_{\alpha}^{h}-I_{\beta}^{h}\right]+\sum_{\substack{\gamma=1 \\ \alpha \neq \gamma \neq \beta}}^{N}\left(\sigma_{\beta \gamma}-\sigma_{\alpha \gamma}\right) I_{\gamma}^{h}\right]
$$

with $I_{\alpha}^{h}=\nabla_{h}^{2} \phi_{\alpha}^{h}+\frac{\pi^{2}}{\eta^{2}} \phi_{\alpha}^{h}$. In order to quickly identify grid points that do not require any calculation, grid points are tagged as interface if they contain more than one phase fields. Any non-interface grid points neighboring an interface grid point are tagged as a near-interface grid points. In a single time step the interface between phase fields can move only to grid points that are tagged as near-interface and not to untagged grid points. This allows the reduction of unnecessary computations.

\subsection{Driving-force Averaging}

As mentioned in section 2.2 we need to ensure the correct phase field profile across the interface an averaging procedure for the driving force is implemented in the OpenPhase. During this procedure the driving force is averaged twice in each grid point over a set of points

$$
S_{\alpha \beta}^{R}\left(x_{h}\right)=\left\{y_{h} \in \Omega_{h} \mid\left(R-\left\|y_{h}-x_{h}\right\|_{2}\right) \phi_{\alpha}\left(y_{h}\right) \phi_{\beta}\left(y_{h}\right)>0\right\},
$$

that is the intersection of a sphere with radius $R=\frac{2}{3} \eta$ and the interface between phases $\alpha$ and $\beta$. In the first step the average

$$
\Delta \bar{g}_{\alpha \beta}^{0}\left(x_{h}\right)=\frac{\sum_{y_{h} \in S_{\alpha \beta}^{R}\left(x_{h}\right)} \omega_{1}\left(y_{h}\right) \Delta g_{\alpha \beta}\left(y_{h}\right)}{\sum_{y_{h} \in S_{\alpha \beta}^{R}\left(x_{h}\right)} \omega_{1}\left(y_{h}\right)}
$$


is weighted with $\omega_{1}\left(y_{h}\right):=\phi_{\alpha}\left(y_{h}\right)^{2} \phi_{\beta}\left(y_{h}\right)^{2}$, which emphasizes values near the center of the interface. In the second step the average

$$
\Delta \bar{g}_{\alpha \beta}\left(x_{h}\right)=\frac{\sum_{y_{h} \in S_{\alpha \beta}^{R}\left(x_{h}\right)} \omega_{2}\left(y_{h}\right) \Delta \bar{g}_{\alpha \beta}^{0}\left(y_{h}\right)}{\sum_{y_{h} \in S_{\alpha \beta}^{R}\left(x_{h}\right)} \omega_{2}\left(y_{h}\right)}
$$

is calculated using the weight $\omega_{2}\left(y_{h}\right):=\Theta\left(\frac{\phi_{\alpha}}{\phi_{\beta}}-0.5\right) \Theta\left(2-\frac{\phi_{\alpha}}{\phi_{\beta}}\right)$, in which $\Theta($.$) is$ the Heaviside function. This average only uses the values near the center of the interface.

\subsection{Sparse Storages}

As mentioned above the computational effort needed compute all combinations of phase fields can be extremely large as it amount of work scales cubically with the number of phase fields. Considering remarks 2.2.1 and 2.2.2 we can, however, significantly reduce the computational load by skipping calculations that do not make a contribution. In a grid point $x_{h} \in \Omega_{h}$ with $\phi_{\alpha}\left(x_{h}\right)=0$ and $\nabla^{2} \phi_{\alpha}\left(x_{h}\right)=0$ we do not need to consider the contribution of the phase field $\phi_{\alpha}$ to any other phase or changes to the phase $\phi_{\alpha}$ itself. That means we only need to compute updates in regions, in which $\phi_{\alpha}(x) \neq 0$ for at least two phases or $\Delta \phi_{\alpha}(x) \neq 0$ for at least one phase. This region is henceforth called the interface between phases. Also in this calculation we only need to regard the contribution of phases with $\phi_{\alpha}(x) \neq 0$ or $\nabla^{2} \phi_{\alpha}(x) \neq 0$. In addition the utilization of sparse storages allows a significantly reduction of the memory requirement, when handling large numbers of phase fields. In order to store the phase fields $\phi_{\alpha}$ and the interactions $\psi_{\alpha \beta}$ OpenPhase uses vectors of a container, that stores up to two indices and up to two values. In each grid point this vector only stores nodes with non-zero values. Values of zero are implied if no node for a phase field or interaction exits.

Generally, in the majority of grid points the vector consists of only one node, which means we are in the bulk of a phase. Vectors with two nodes will be quite frequent as well, however, grid points in which five or more nodes are needed are very rare. Due to the small data sets a linear search algorithm is used as it is the most efficient in this case. Using these sparse storages OpenPhase can handle an arbitrarily large 
number of different phase fields. Depending on the specific data this storage can be interpreted as symmetric or antisymmetric, which further reduces the amount of data by half the number of possible combinations. Using these sparse storages however makes the use of implicit time stepping schemes impossible.

\subsection{Time Stepping}

As seen in section 2.3 time stepping is conducted by a first order explicit scheme. In order to guarantee stable simulations the time step size $\Delta t_{n}$ has to be limited, for phase field we get the condition

$$
\Delta t_{n} \dot{\psi}_{\alpha \beta}^{\mathrm{GB}}\left(x_{h}, t_{n}\right)<0.1 \quad \forall x_{h} \in X_{h}, 1 \leq \alpha, \beta \leq N
$$

For diffusion of solute elements the step size $\Delta t_{n}$ is limited by

$$
\Delta t_{n} D_{\max }<\frac{1}{4} h^{2}
$$

with the maximum diffusion coefficient $D_{\max }$ in $\Omega$. In the simplest implementation we can select a time step size $\Delta t_{n}$ that fulfills all these conditions and do a time step. However, this can be inefficient if one condition is more restrictive than another. In this case the computational work could be increased severely. Therefore it makes sense to use different time step sizes $\Delta t^{a}$ for different methods. In order to align properly we enforce step sizes $\Delta t^{a}$ for a method $a$ with

$$
M_{a} \Delta t^{a}=\Delta t^{\max }
$$

with an integer $M_{a}$ and the maximum step size $\Delta t^{\max }$ among all methods. 


\section{The Lattice-Boltzmann Method}

In order to simulate fluids OpenPhase utilizes the Lattice Boltzmann method, a simple and powerful tool for the modeling of complex fluid systems [27, 28, 29]. The method calculates the evolution of the particle velocity distributions $f_{i}$ in two steps, collision

$$
f_{i}^{c}(x, t)=f_{i}(x, t)-\frac{1}{\tau}\left[f_{i}(x, t)-f_{i}^{e q}(x, t)\right]
$$

and streaming

$$
f_{i}\left(x+e_{i} \Delta t, t+\Delta t\right)=f_{i}^{c}(x, t) .
$$

The Lattice-Boltzmann method is a numerical scheme that is derived by simplifying the Boltzmann equation

$$
\left(\partial_{t}+v \cdot \nabla_{x}+\frac{1}{m} F \cdot \nabla_{v}\right) f(x, v, t)=\Omega(f),
$$

for a particle density function $f(x, v, t)$ with position $x$, velocity $v$, time $t$, mass $m$ and an external force $F$ and collision integral $\Omega(f)$. Intermediate steps are the lattice gas automata that discretize space, time and the particle velocities [30]. They use a regular lattice of nodes $x_{h}$ and Boolean variables $n_{i}\left(x_{h}, t_{n}\right)$ for $i=1, \ldots, m$ that indicates whether a node contains a particle with the velocity $e_{i}$. Ignoring any external force $f$ the evolution equation becomes

$$
n_{i}\left(x_{h}+e_{i}, t_{n}+1\right)=n_{i}\left(x_{h}, t_{n}\right)+\Omega_{i}\left(n\left(x_{h}, t_{n}\right)\right.
$$

with the collision operator $\Omega_{i}\left(n\left(x_{h}, t_{n}\right)\right.$, that describes the interaction of particles at a node according to a scattering rule [28]. For the Lattice-Boltzmann method the Boolean variables $n_{i}\left(x_{h}, t_{n}\right)$ are replaced by particle distribution function $f_{i}=\left\langle n_{i}\right\rangle$, that allow us to define the density $\rho$ of a fluid by

$$
\rho=\sum_{i=1}^{m} f_{i}
$$


and the momentum $\rho v$ by

$$
\rho v=\sum_{i=1}^{m} f_{i} e_{i}
$$

with the velocity $v \in \mathbb{R}^{d}$ and the particle velocity $e_{i} \in \mathbb{R}^{d}$. The kinetic equation is given by

$$
f_{i}\left(x+e_{i} \Delta x, t+\Delta t\right)=f_{i}(x, t)+\Omega_{i}(f(x, t),
$$

with the collision operator $\Omega_{i}$ that is required to satisfy

$$
\begin{aligned}
\sum_{i=1}^{m} \Omega_{i} & =0, \\
\text { and } \sum_{i=1}^{m} \Omega_{i} e_{i} & =0,
\end{aligned}
$$

to conserve mass and momentum. Taylor expansion in time and space of equation 3.7 gives

$$
\epsilon\left[\partial_{t} f_{i}+\partial_{e_{i}} f_{i}+\epsilon\left(\frac{1}{2} \partial_{e_{i}} \partial_{e_{i}} f_{i}+\partial_{e_{i}} \partial_{t} f_{i}+\frac{1}{2} \partial_{t}^{2} f_{i}\right)\right]=\Omega_{i}
$$

Expansion of the particle distribution function $f_{i}$ about the local equilibrium distribution gives

$$
f_{i}=f_{i}^{\mathrm{eq}}+\epsilon f_{i}^{(1)}+\epsilon^{2} f_{i}^{(2)}+O\left(\epsilon^{3}\right),
$$

which are subject to the constraints

$$
\begin{gathered}
\rho=\sum_{i=1}^{m} f_{i}^{\mathrm{eq}}, \\
\rho v=\sum_{i=1}^{m} f_{i}^{\mathrm{eq}} e_{i},
\end{gathered}
$$

and

$$
\begin{aligned}
\sum_{i=1}^{m} f_{i}^{(k)} & =0, \\
\sum_{i=1}^{m} f_{i}^{(k)} e_{i} & =0 .
\end{aligned}
$$


With non-equilibrium part $f_{i}^{\text {neq }}=f_{i}^{(1)}+\epsilon f_{i}^{(2)}+O\left(\epsilon^{2}\right)$ the collision operator $\Omega_{i}$ can be expanded to

$$
\begin{aligned}
\Omega_{i}(f) & =\Omega_{i}\left(f^{\mathrm{eq}}\right)+\epsilon \sum_{j=0}^{M} M_{i j} f_{j}^{(1)} \\
& +\epsilon^{2}\left(\sum_{j=0}^{M} M_{i j} f_{j}^{(2)}+\sum_{k=0}^{M} \sum_{j=0}^{M} \partial_{f_{k}} M_{i j} f_{j}^{(1)} f_{k}^{(1)}\right)+O\left(\epsilon^{3}\right), \\
& =\Omega_{i}\left(f^{\mathrm{eq}}\right)+\epsilon \sum_{j=0}^{M} M_{i j}\left(f_{j}^{(1)}+\epsilon f_{j}^{(2)}+O\left(\epsilon^{2}\right)\right)+O\left(\epsilon^{3}\right),
\end{aligned}
$$

with the collision matrix $M_{i j}:=\partial_{f_{j}} \Omega_{i}\left(f^{\mathrm{eq}}\right)$. Using $\Omega_{i}\left(f^{\mathrm{eq}}\right)=0$, we get

$$
\frac{\Omega_{i}(f)}{\epsilon}=\frac{M_{i j}}{\epsilon}\left(f_{j}-f_{j}^{\mathrm{eq}}\right) .
$$

Using $M_{i j}=-\frac{1}{\tau} \delta_{i j}$ and equation 3.7 we obtain the LBGK equation

$$
f_{i}\left(x+e_{i} \Delta x, t+\Delta t\right)=f_{i}(x, t)-\frac{\left(f_{j}-f_{j}^{\mathrm{eq}}\right)}{\tau} .
$$

The relaxations time

$$
\tau=3 \nu \frac{\Delta t}{(\Delta x)^{2}}+\frac{1}{2}
$$

depends on the kinematic viscosity $n u$ and the time and length scales $\Delta t$ and $\Delta x$ [29].

\subsection{Decaying Taylor-Vortex}

In order to verify the validity of the implementation, a decaying Taylor-vortex simulated. The test has been adapted from [31]. The analytical solution in this case is given by

$$
v(x, t)=\left(\begin{array}{c}
-u_{0} \sqrt{\frac{k_{j}}{k_{i}}} \cos \left(k_{i} x_{i}\right) \sin \left(k_{j} x_{j}\right) \exp \left(-\frac{t}{t_{D}}\right) \\
u_{0} \sqrt{\frac{k_{i}}{k_{j}}} \sin \left(k_{i} x_{i}\right) \cos \left(k_{j} x_{j}\right) \exp \left(-\frac{t}{t_{D}}\right. \\
0
\end{array}\right),
$$


with the decay time

$$
t_{D}=\frac{1}{\nu\left(k_{i}^{2}+k_{j}^{2}\right)},
$$

The Lattice-Boltzmann populations $f_{i}$ at $t=0$ are initialized using both equilibrium and non-equilibrium contributions

$$
f_{i}=f_{i}^{\mathrm{eq}}+f_{i}^{\mathrm{neq}}
$$

with the first order approximation

$$
f_{i}^{n e q} \approx f_{i}^{1}=\frac{w_{i} \tau}{2 c_{s}^{2} \nu} Q_{i}: \sigma
$$

The deviatoric stress tensor $\sigma$ at time $t=0$ is then given by

$$
\begin{aligned}
& \sigma(x, 0)= \\
& {\left[\begin{array}{ccc}
z_{0} \sqrt{k_{i} k_{j}} \sin \left(k_{i} x_{i}\right) \sin \left(k_{j} x_{j}\right) & z_{0}\left(\sqrt{\frac{k_{i}^{3}}{k_{j}}}-\sqrt{\frac{k_{j}^{3}}{k_{i}}}\right) \cos \left(k_{i} x_{i}\right) \cos \left(k_{j} x_{j}\right) & 0 \\
z_{0}\left(\sqrt{\frac{k_{j}^{3}}{k_{i}}}-\sqrt{\frac{k_{i}^{3}}{k_{j}}}\right) \cos \left(k_{i} x_{i}\right) \cos \left(k_{j} x_{j}\right) & z_{0} \sqrt{k_{i} k_{j}} \sin \left(k_{i} x_{i}\right) \sin \left(k_{j} x_{j}\right) & 0 \\
0 & 0 & 0
\end{array}\right]}
\end{aligned}
$$

with the coefficient

$$
z_{0}=2 \rho \nu u_{0}
$$

The matrix $Q_{i}$ is given by

$$
Q_{i}=c_{i} c_{i}^{t}-c_{s}^{2} \mathbb{I}
$$

with the identity matrix $\mathbb{I}$. Figure 3.1 shows the second order convergence of the error in the $L_{\infty}$-norm for increasing grid resolution. A kinematic viscosity $\nu=0.18$ and a relaxation time $\tau=1.04$ give the best result.

\subsection{The Exact Difference Method}

Different body forces such as gravity, buoyancy, drag or the Shan-Chen Force for liquid-gas two phase system $[32,33]$ can act on the fluid. All forces acting on the 


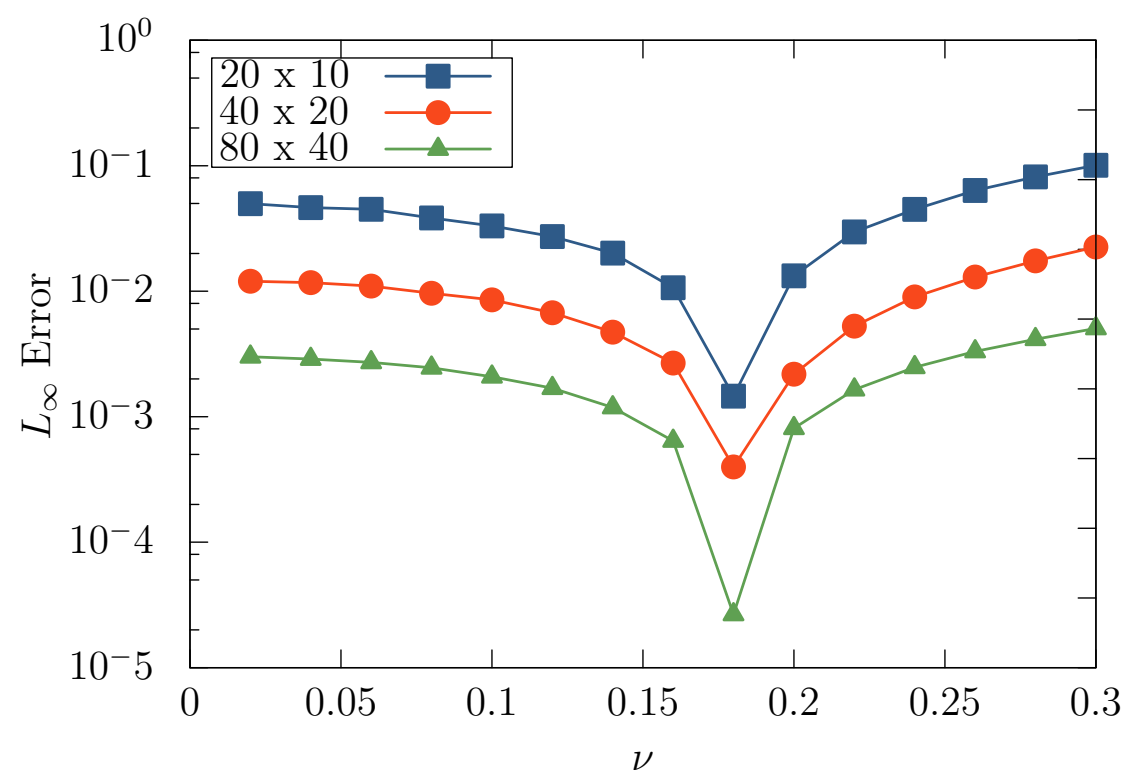

Figure 3.1: $L_{\infty}$-error depending on the kinematic viscosity $\nu$.

fluid are combined in a total local body force $F=F_{1}+F_{2}+\ldots$, where $F_{1}$ and $F_{2}$ are different force contributions. Following [34] the evolution equation 3.20 is expanded to

$$
f_{i}\left(x+e_{i} \Delta x, t+\Delta t\right)=f_{i}(x, t)-\frac{\left(f_{j}-f_{j}^{\mathrm{eq}}\right)}{\tau}+\Delta f_{i},
$$

with the difference of the equilibrium distributions

$$
\Delta f_{i}=f_{i}^{\mathrm{eq}}\left(\rho, u+\frac{F \Delta t}{\rho}\right)-f_{i}^{\mathrm{eq}}(\rho, u) .
$$

\subsection{Fluid-Solid-Interaction}

Two kinds of fluid-solid interaction have to be considered; one is the momentum exchange between the fluid and solid bodies submerged in the fluid. Another is the incorporation of wetting properties of the solid in multiphase settings. Regarding the momentum exchange a modification of the streaming step is used following [35]. 
Grid points $x_{h}$ are marked as obstacles if in this grid point no fluid is present, in conjunction with the phase field method this means

$$
\phi_{i}\left(x_{h}\right)=0 \text { for all } i \in I_{\text {Fluid }}
$$

with the index $I_{\text {Fluid }}$ that contains all fluid phases. This means grid points in the interface between solid and fluid are not marked as obstacles. The update to particle populations $f_{i}\left(x_{h}, t\right)$ at grid point $x_{h}$ is modified to include the bounce back at obstacle grid points $x_{h}+e_{i}$, denoting with $e_{\bar{i}}$ the opposite direction to $e_{i}$ we have

$$
f_{\bar{i}}\left(x_{h}, t+1\right)=f_{i}\left(x_{h}, t\right)+2 w_{i} e_{\bar{i}} v_{s}\left(x_{h}+e_{i}\right),
$$

with the local solid velocity $v_{s}\left(x_{h}+e_{i}\right)$, that includes the translational and angular velocities. The fluid also exerts a force on the solid body given by

$$
F=2\left(f_{i}+w_{i} e_{i} v_{s}\left(x_{h}+e_{i}\right)\right) e_{i}
$$




\section{High-Performance Computing}

Numerical simulations of complex physics can require significant amounts of computational work that would take an unreasonable long time if computed by a single processing unit. Especially in the recent years the performance per processing unit has become stagnant $[36,37]$. In order to reduce the wall time of simulations the distribution of work among many processing units is required. Multicore-processors consist of multiple processing units that are located on a single component. Multiple of these Multicore-processors can be assembled on a single node with access to shared memory. Multiple of these nodes that do not share their memory can be combined in a network.

Figure 4.1 shows a schematic overview of a fat-tree network, in which the bandwidth increases with each additional level of network switches. At the leaf level each switch is connected to several nodes. Each node can consist of multiple sockets with their own local memory, which are connected by a distributed-shared memory (DSM). While the each core can access any memory location on the same node, the memory access of cores that are directly connected by a bus to their local memory is significantly faster.

\subsection{Parallel Programming Models}

Depending on how individual parallel computational entities can interact with each other, different parallel programming models are used. If all computational entities share the same address space the shared-memory parallelism model can be used. If they do not share the same address space, distributed-memory parallelism has to be used. 


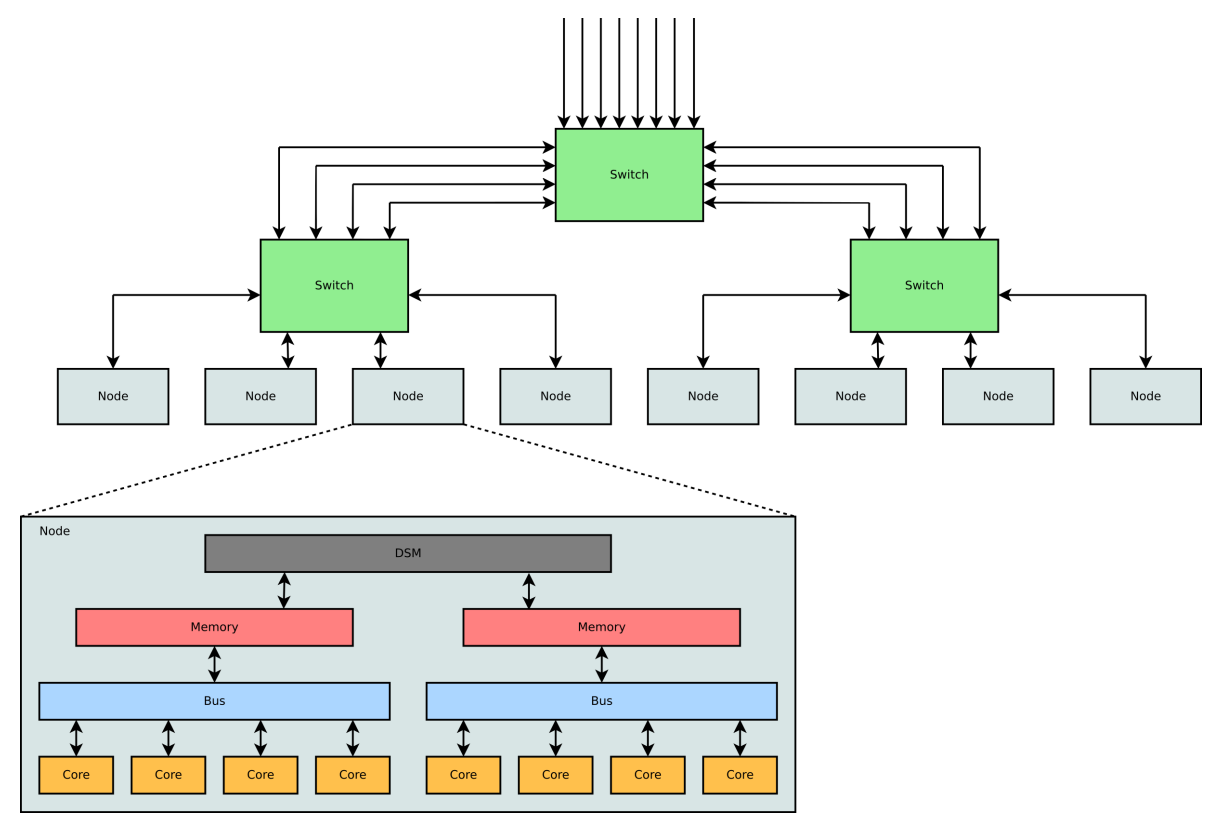

Figure 4.1: Schematic overview of a fat-tree architecture.

\subsubsection{Distributed-Memory Parallelism}

Distributed-memory parallelism uses processes, instances of a computer program, that use their own private address space. That means each process can only access and manipulate its own associated memory. In order to synchronize data between processes explicit communication of data from one process to another is required. The Message Passing Interface (MPI) is the standard that has been used to enhance OpenPhase with distributed-memory parallelism. The implementation OpenMPI 1.6.3 has been used on Vulcan, the parallel cluster located at ICAMS, and Parastation MPI 5.1.5-1 on Jureca, which is based on MPICH v3 and implements MPI 3.0 .

\subsubsection{Shared-Memory Parallelism}

In shared-memory system multiple threads, sequences of instructions, can be executed simultaneously using a shared address space. Any thread can own private 
data, but it can also access any shared data. Therefore special care needs to be taken when manipulating data in order to avoid so-called race conditions, which can occur when the order of data access is not guaranteed to be in the correct order. Synchronization and semaphores can be used to maintain the correct order of operations. There are multiple APIs for shared-memory multi-threaded programming such as OpenMP ([38]), TBB and CILK ([37]). As of the writing of this work OpenPhase uses exclusively OpenMP for shared-memory parallelism. Threads generally create a much smaller overhead than processes and can easily be created and destroyed during the execution of a program.

\subsubsection{Hybrid-Parallelism}

Hybrid-parallelism is the combination of distributed- and shared-memory parallelism. As threads generally require a much smaller overhead than processes it makes sense to use threads instead of processes when possible. In practice however many applications yield a higher performance with pure distributed parallelism. Reasons for this behavior can be diverse. As some MPI implementation only allow thread single communication, i.e. only one thread within each process can initiate interprocess communication at the same time, less computation resources participate in communication, which can slow down the application.

\subsection{Estimating Speedup}

A general goal of high performance computing is to use more and faster resources towards the solution of computational problems. Amdahl's law [39] gives an estimate on the speed up $S(N)$ of a parallel program with $N$ processors:

$$
S_{\text {Amdahl }}(N)=\frac{1}{\frac{P}{N}+S},
$$



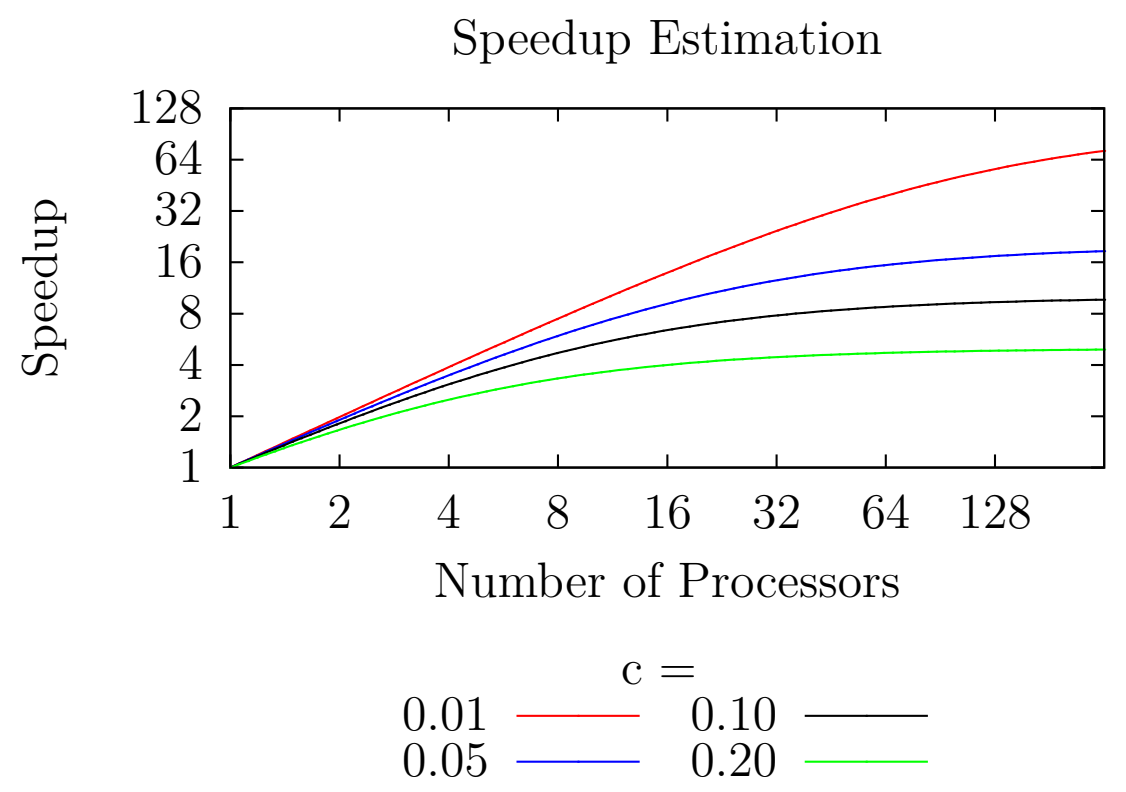

Figure 4.2: Estimated Speedup due to Amdahl's Law.

where $P$ is the portion of work, that can be computed in parallel, and $S=1-P$ the portion that is computed sequentially. If we have a load imbalance $c$, this imbalance contributes to the amount of sequential work, such that

$$
S=\frac{w_{\max }}{\tilde{W}}-1 \leq(1+\gamma)-1=c
$$

and thus

$$
S_{A m d a h l}(N) \geq \frac{1}{\frac{1-c}{N}+c}
$$

In figure 4.2 we can see the worst case speedup for different values of maximal load imbalance $c$. Here we see that the load imbalance strongly influences the speedup and the number of processors suitable for the problem. E.g. if we have a $10 \%$ load imbalance it makes no sense to use more than eight processors.

However, if we fix the amount of time and look at the increase of the problem size, we can apply Gustafson's law [40]

$$
S_{\text {Gustafson }}(N)=N-c(N-1) \text {. }
$$




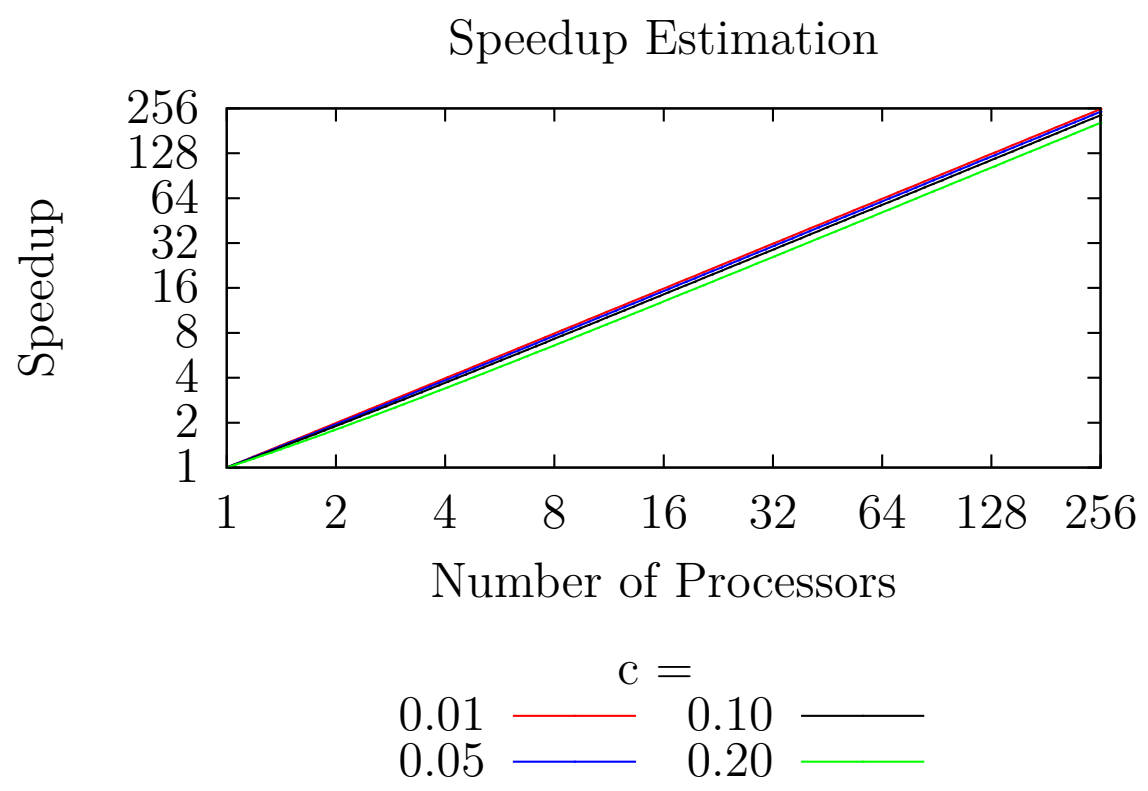

Figure 4.3: Estimated Speedup due to Gustafson's Law.

In figure 4.3 we see the estimated speedup for different load imbalances $c$ for an increasing total workload. We see a rather good efficiency for small imbalances $c$ in figure 4.4. That means as long as load imbalances are small proper weak scaling capabilities can be expected. In following the focus will be on the weak scaling performance as the goal is increase system sizes in order to obtain physically relevant result by ruling out size effects.

\subsection{Load-balancing}

The goal of load-balancing is to minimize the wall time of a parallel program by removing load-imbalances between processes. In order to achieve that goal the costs of data communication and migration as well as the cost of load-balancing itself have to be considered in addition to the load-balance between processes. In other words we want to minimize the total time

$$
T=T_{\mathrm{LB}}+T_{\text {Migration }}+\sum_{\alpha}\left[\max _{p \in P} T_{\text {Work }}^{\alpha, p}+\max _{p \in P}^{p} T_{\text {Comm }}^{\alpha, p}\right]
$$




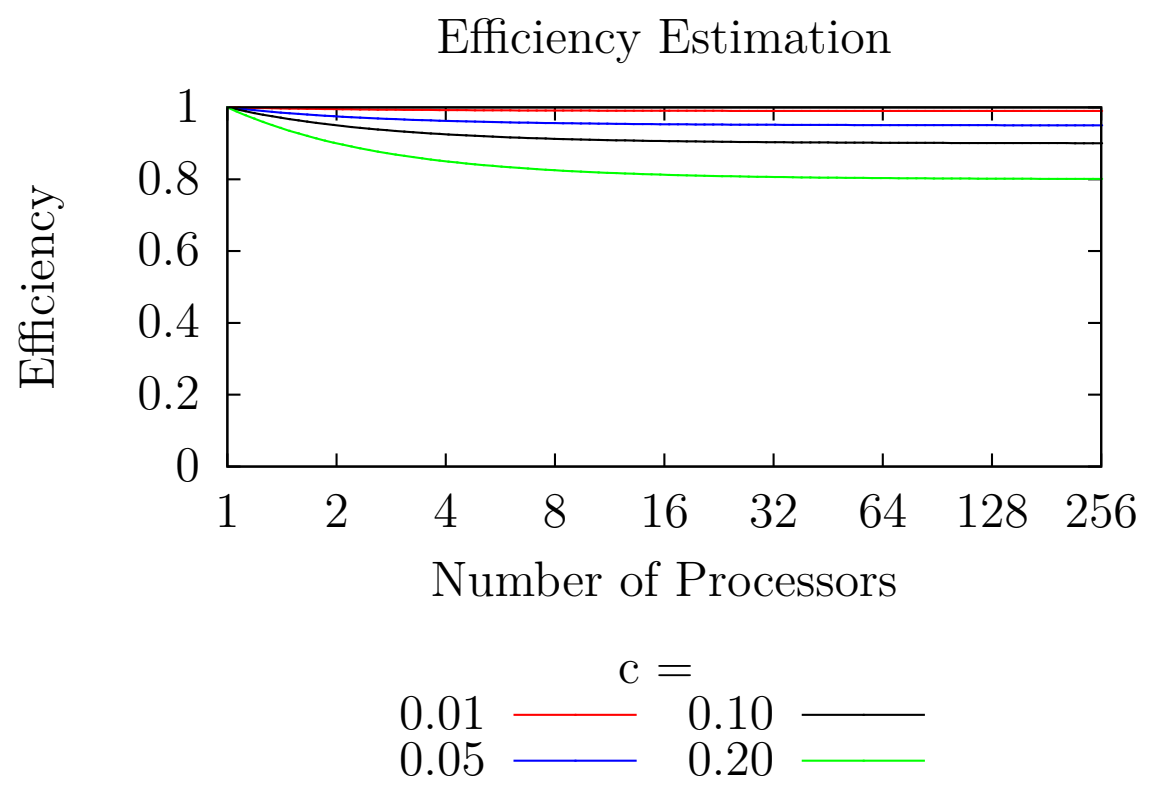

Figure 4.4: Estimated Efficiency due to Gustafson's Law.

that consists of the time $T_{\mathrm{LB}}$ for the load-balancing scheme itself, the time $T_{\text {Migration }}$ for data migration as well as the time $T_{\text {Work }}^{\alpha, p}$ spend in work segments on process $p \in P$ and time $T_{\text {Comm }}^{\alpha, p}$ for communication of data after the work segment $\alpha$ for process $p \in P$, such as halo exchanges or reductions of global properties. In each work segment only the maximum amount of time required among the processes $p \in P$ is relevant for the total time. It depends on the specific problem which contribution to the total is the most significant and accordingly different load-balancing schemes can be useful. The load imbalance factor $\gamma^{\alpha}$ of a work section $\alpha$ is given by

$$
\gamma^{\alpha}:=\frac{\left.\max _{p}() T_{\text {Work }}^{\alpha, p}\right)}{\tilde{T}_{\text {Work }}^{\alpha}}
$$

where $\max _{p}\left(T_{\text {Work }}^{\alpha, p}\right)$ is the time used on the work section by the process, that used the longest amount of time and $\tilde{T}_{\text {Work }}^{\alpha}$ is the average time among the processes to complete the work section. 


\subsection{Other Parallel Phase Field Implementations}

Multiple attempts have been conducted to parallelize phase field simulations in order to treat large systems [18, 19] including the impressive results in [20], which won the Gordon Bell prize in 2011 for scalability. In [41] a parallel implicit multi grid solver is presented. However, due to the implicit solution the solver is limited to a small amount of different phase fields as the utilization of sparse storages is not feasible in this case. Also in explicit solvers the amount of different phase fields is limited [18, 20, 42], which allows implementations with minimal load imbalance and removes the need for adaptive load-balancing, which is as mentioned above a significant obstacle for scalable parallel solvers. As [19] points out the parallel efficiency and the load-balancing capabilities of the multiphase field software PACE3D [21] is limited by the lack of a three-dimensional domain decomposition. The well-known multiphase field software Micress $\mathbb{R}[22]$ does not support distributed-memory parallelization. 



\section{Parallelization}

As presented in [1] and [3] OpenPhase has been extended with a hybrid parallelization that uses both MPI and OpenMP. The concepts of the parallelization and the load-balancing scheme will be recalled and expanded.

As commonly utilized in parallel computing, the computational domain $\Omega$ is divided into smaller sub-domains $\Omega_{i}$ that are assigned to the processors in order to share the workload. For reasons of simplicity only rectangular sub-domains are used, as these allow for a simple description by their position and size in each dimension. Henceforth these sub-domains will be referred to as blocks $B_{i}:=B_{\Omega_{i}}$; the computation on these blocks is further parallelized using the thread-based OpenMP.

For simple finite-difference problems load-imbalances are negligible, as the same stencil operation is performed in every grid point. As discussed in section 2.5 the computational cost depends on the number of active phase fields in a grid point when using the multiphase field method with sparse storages. Therefore, the computational work at the interface between phases is increased and thus can lead to heterogeneous load distributions. As the performance of a parallel program is limited by its slowest processor a load-imbalance can severely restrict its performance. An over-decomposition of the domain is used to create more blocks than processes, which allows a graph-partitioner to assign blocks to processes in a way that provides a sufficient load-balance and reduces communication between processes. Communication of data between blocks on different processes is implemented using MPI and the data exchange between blocks on the same process in implemented as a simple copy operation.

Some graph-partitioners such as METIS or ParMETIS [43] support multi-constraint graph-partitioning, which can be used to balance problems with multiple sections that are separated by synchronization points, in which data is communicated. Other graph-partitioning tools such as PT-SCOTCH [44] only support single-constraint partitioning. In this work only single-constraint partitioning will be considered. 
Therefore it is required to have all computation within one time step in a single section that is not interrupted by communication. However, performing a stencil operation invalidates grid points at the boundary, for which the stencil could not access grid points with valid data. The canonical approach would be to update the ghost data after every sweep with a stencil operation. This would, however, introduce a synchronization point. Using a wide halo pattern as presented in [45] allows us to remove unnecessary synchronization points as seen in figure 5.2. In figure 5.4 the concept of the wide halo is depicted. The size of the halo is increased such that information is still valid when all modules hit a common synchronization point; as such the size of the halo depends on the particular problem.

If a small number of large blocks are used, it can be difficult or impossible for the graph-partitioner to find a suitable load-balance. If, however, a large number of small blocks are used, additional overhead is introduced by the halo that increases the total amount of work. In the following a framework for the control of blocks sizes is presented. A dynamic scheduling in the OpenMP parallelization on each block easily allows for load-balancing among the threads for each MPI-process.

\subsection{Domain-Decomposition}

As commonly utilized in parallel computing, the computational domain is divided into sub-domains that are distributed among the processors to share the workload. For reasons of simplicity only rectangular sub-domains will be considered, as these can be described by just a position and its dimensions. Henceforth these subdomains will be referred to as blocks. As mentioned in section 2.5 we use sparse storages for the data and only compute updates on phase fields when necessary. This leads to heterogeneous load distribution in the computational domain, which could lead to load imbalances in a parallel setting. An over-decomposition of the domain, i.e. more blocks than processes, enables us to assign blocks to processes and achieve a suitable load-balance. 

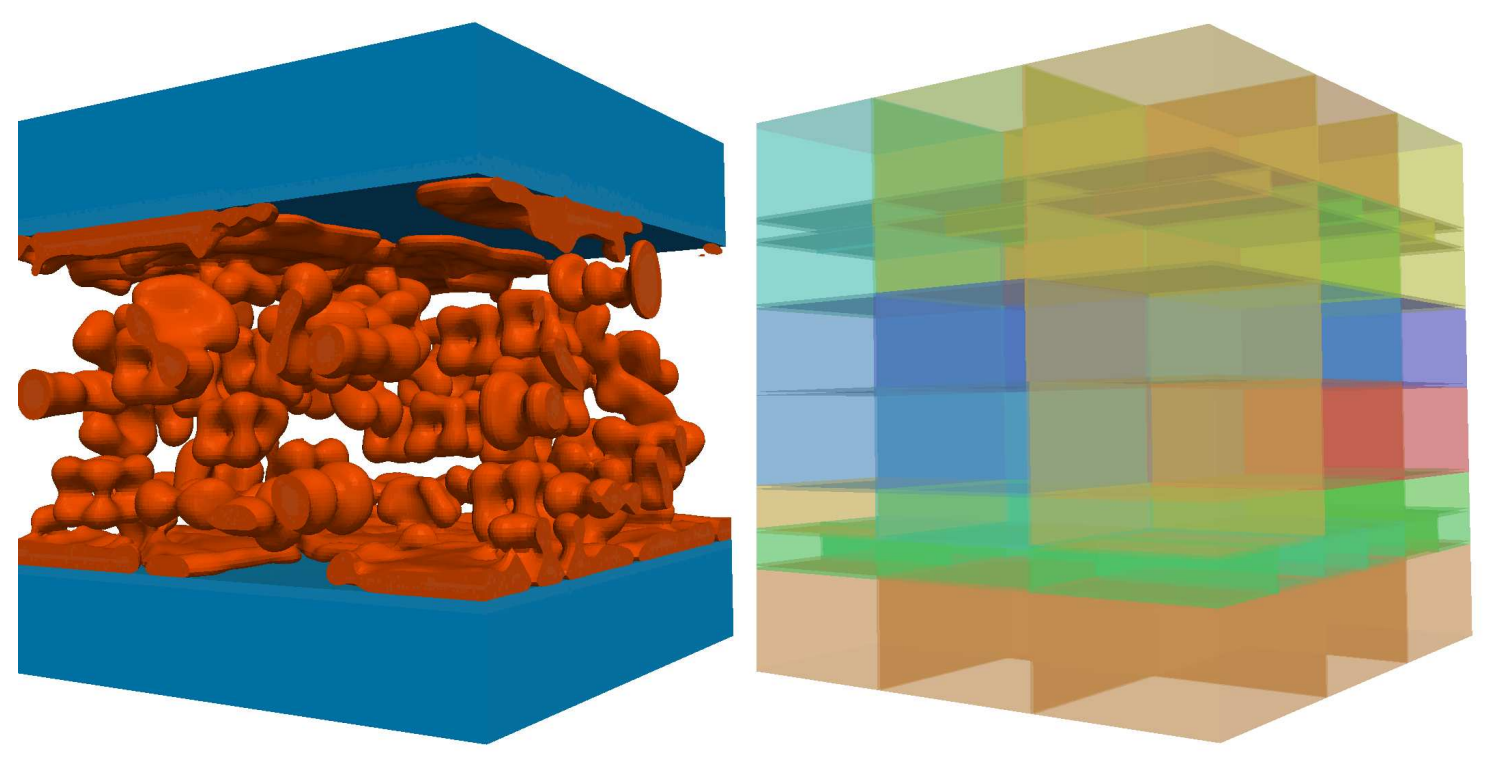

Figure 5.1: Simulation of interdendritic solidification of a $\mathrm{Mg}-\mathrm{Al}$ alloy. The corresponding domain decomposition is shown on the right-hand side. Different colors indicate different process ranks.

\subsubsection{Wide Halo}

In order for this to yield beneficial results, it is necessary to have a balanced load between each synchronization point. With our data decomposition approach this means that we need to avoid synchronization points within a single time step. Performing a stencil operation invalidates grid points at the boundary, for which the stencil could not access grid points with valid data. The canonical approach would be to update the ghost data after every sweep with a stencil operation. This, however, is problematic as it introduces a synchronization point, which interferes with load-balancing. Using a wide halo pattern as presented in [45] allows us to remove unnecessary synchronization points as seen in figure 5.2. This is essential for a proper balance of the computational load.

In figure 5.4 the concept of the wide halo is depicted. The size of the halo is increased such that information is still valid when all modules hit a common synchronization point; as such the size of the halo depends on the particular problem. 


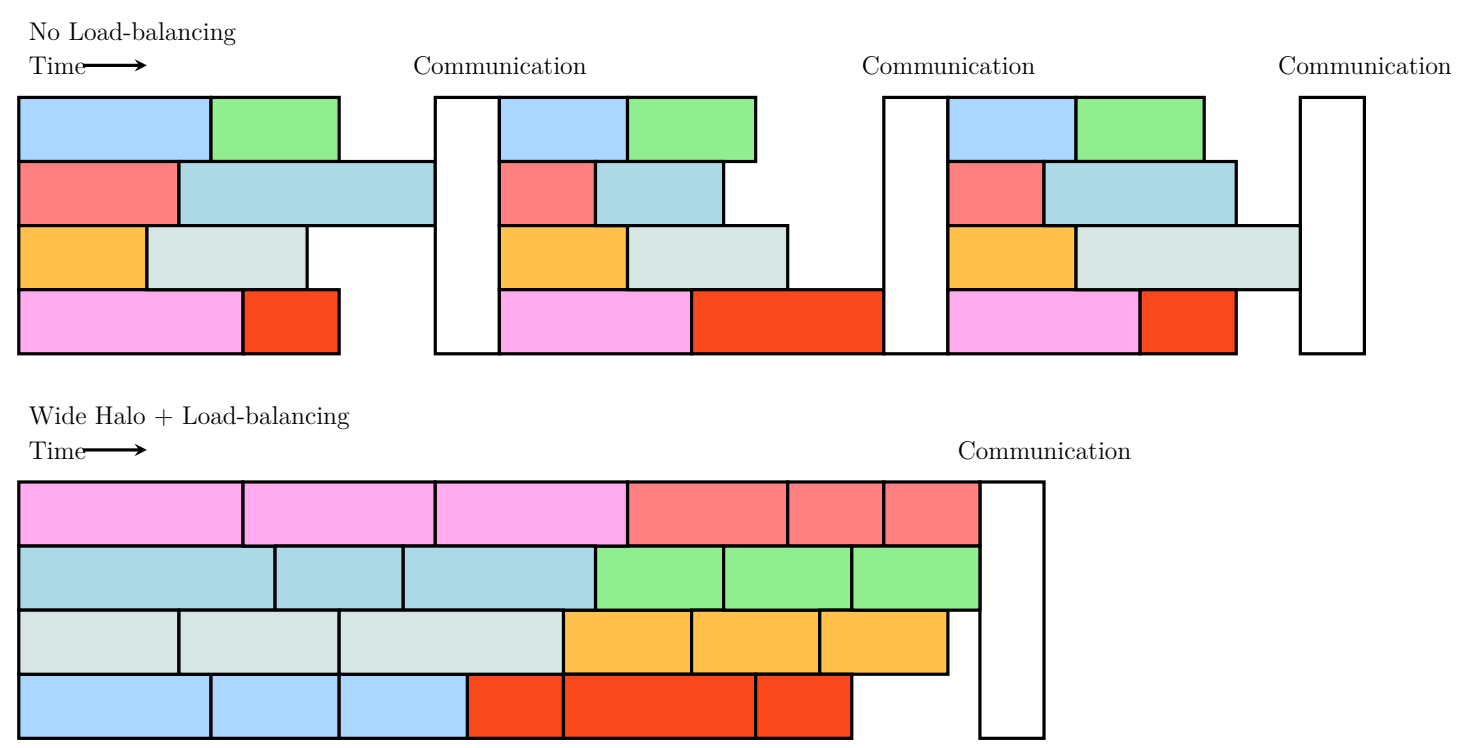

Figure 5.2: This shows an example for a scheduling with four processes and two subdomains on each process. Each work segment on the same sub-domain as indicated by the same color has to be assigned to the same process. The schedule at the top shows a parallelization with multiple synchronization points per time step, while the bottom shows a parallelization with load balancing and removed synchronization points.

\subsubsection{Communication Pattern}

After every time steps the old invalid principal data values in the halo of each block, i.e. data that cannot be derived within a time step, need to be updated with valid data located on neighboring blocks. Each block has a list of neighbors, that is all blocks for which the halo of a block intersects with the interior domain of another block, it knows the size and coordinates of the neighbor blocks and as well as the process ID the blocks are located on. The neighborship relation is symmetric, that means if block $\mathrm{A}$ is a neighbor block $\mathrm{B}$, than block $\mathrm{B}$ is a neighbor of block $\mathrm{A}$. In order to communicate data each process creates a send buffer for each other process it needs to communicate with. The process will concatenate data from its blocks if they need to send data to blocks on the same process. Note a block can send data to multiple blocks located on the same process.

As mentioned in section 2.5 we use sparse storages that can change the amount of 

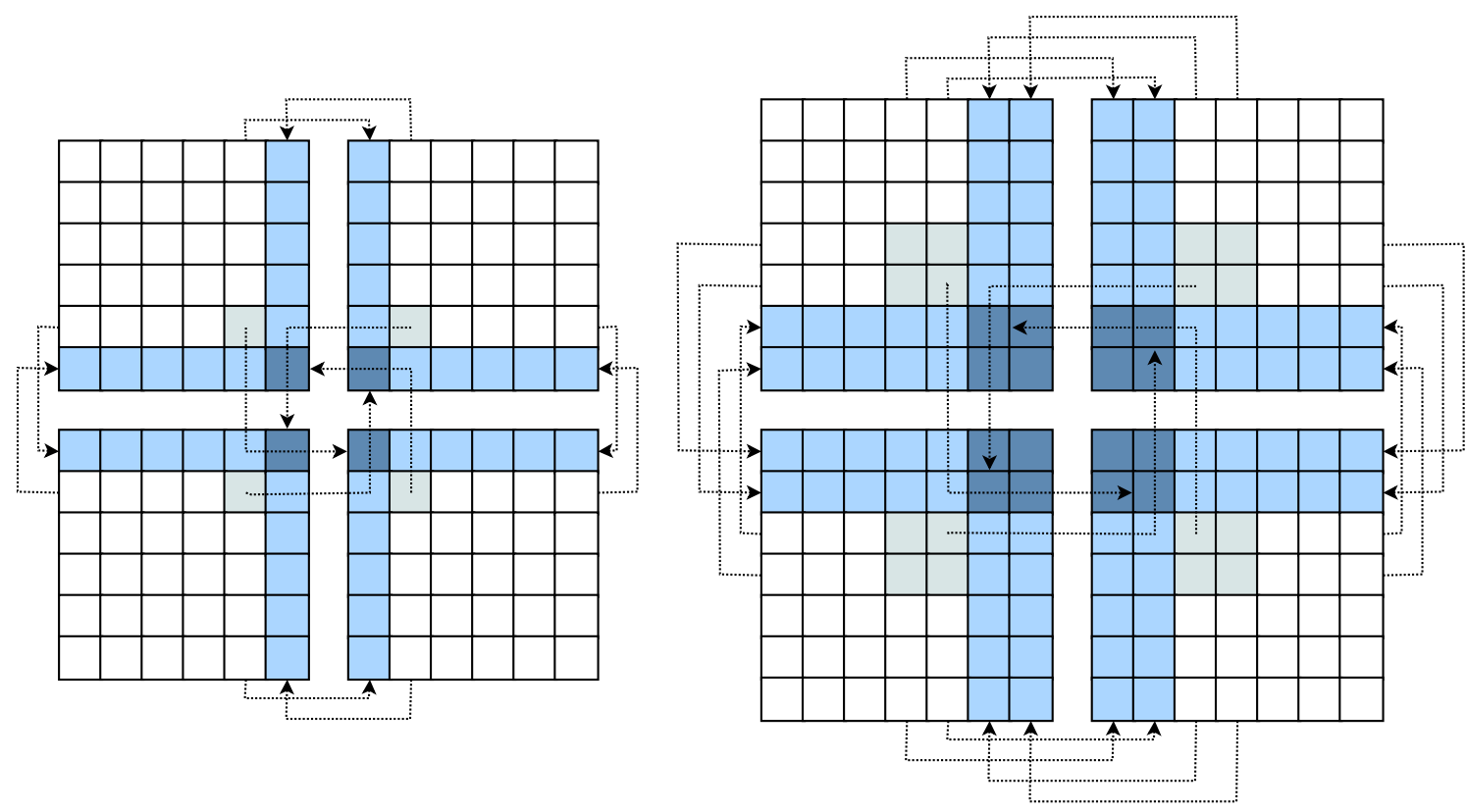

Figure 5.3: The left-hand side shows the needed communication with one layer of ghost cells. Communication with two layers of ghost cells is presented on the righthand side.

data in each grid point, therefore we cannot determine the size of the send buffer by simply counting the number of grid points. One dangerous option is to use a size of the receive buffer, that is large enough to fit any amount of data. This approach can either increase the memory requirements significantly or fail because for one particular problem the buffer turns out to be too small. Therefore the data is sent in two steps. The first is the communication of the send buffer sizes to the receiving processes; the second is the communication of the actual data. Algorithm 1 shows how the halo exchange is implemented. map refers to the std::map, which is a binary map that stores elements by key and value and only creates an entry the first time a key is accessed. Therefore if no data is put to a specific process is put into a buffer, the key will not exist and no communication with that process will occur. 


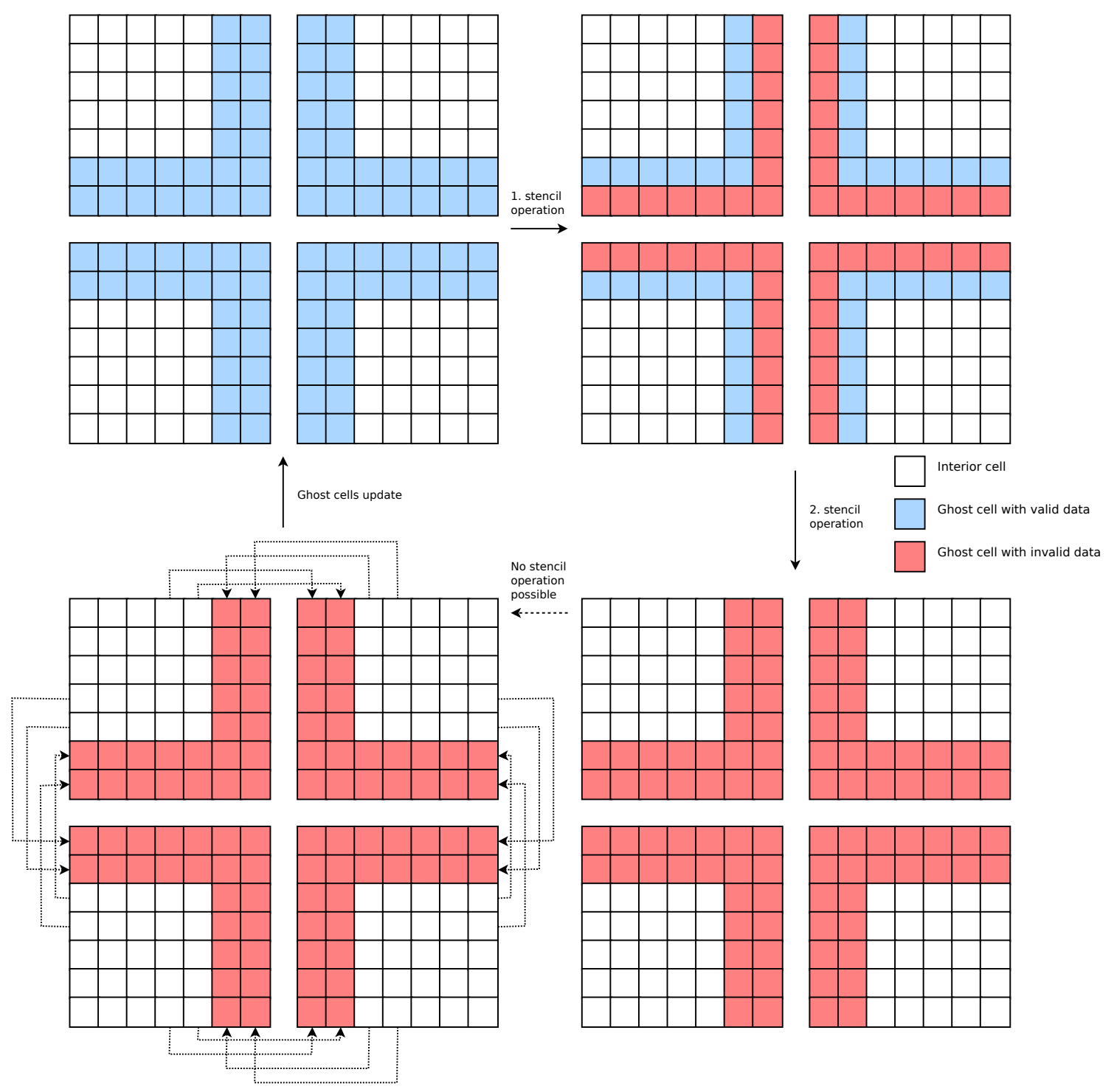

Figure 5.4: With two layers of ghost cells two stencil operations are possible before the ghost data needs to be synchronized. More layers of ghost cells allow more stencil operation without interruption by communication. 


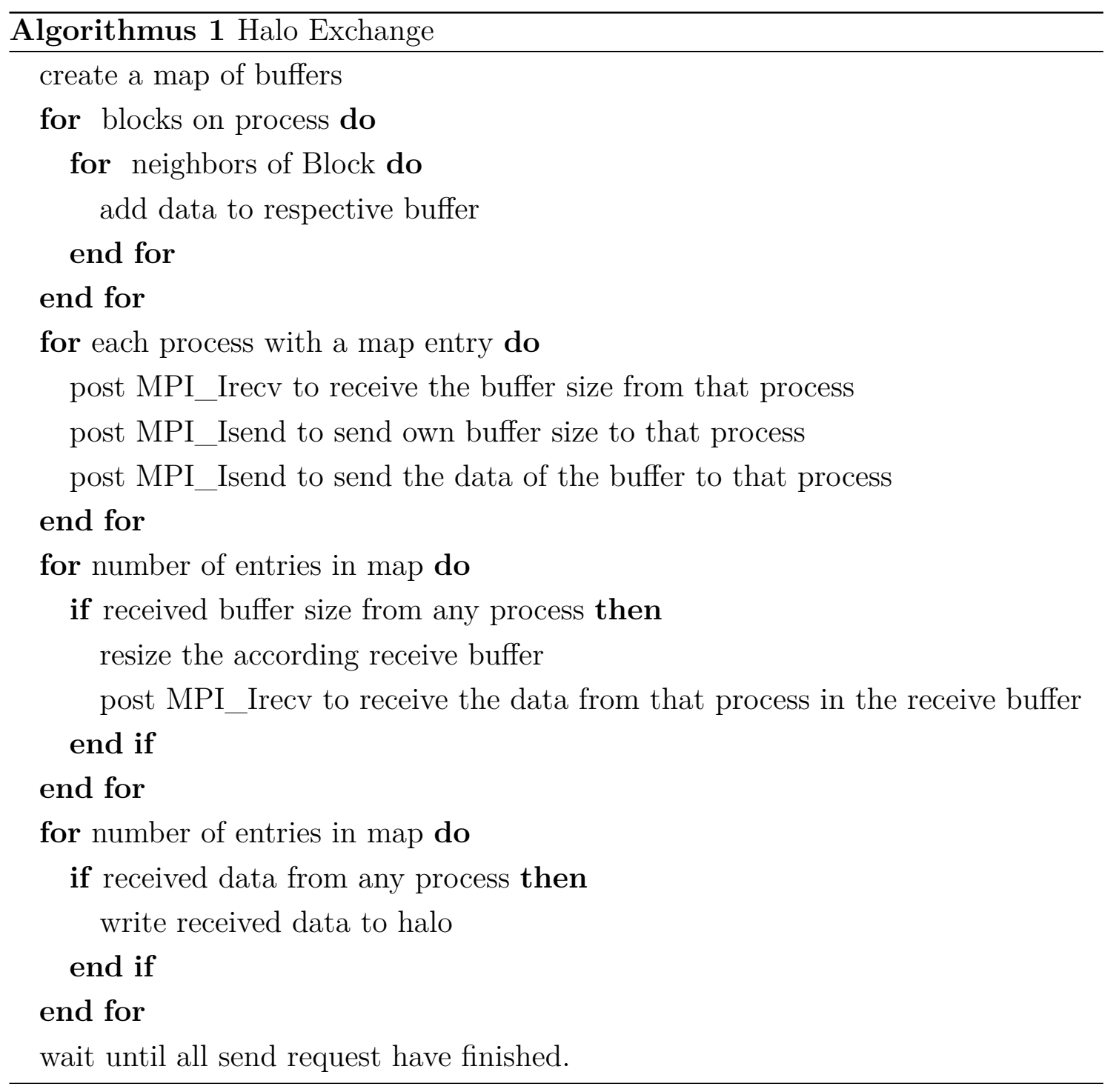

\subsection{Load-balancing}

For homogeneous load distributions simple domain decompositions are feasible, i.e. each process works on one equally sized sub-domain. However, due to the sparse storages (see section 2.5 a heterogeneous load distribution is possible. In this case load-balancing helps to achieve an increased performance as idle times of the processors are reduced. As the load distribution can evolve during a simulation the 
work assignment needs to be adapted during the runtime of the program. Thus, the computational domain is over-decomposed into number of sub-domains larger than the number of available processors. As these sub-domains are rectangular, we will subsequently call them blocks. The load-balancing strategies, that were discussed in [1], are used to assign the blocks to process in a way such that the maximum load on the processes is minimized. For completion we repeat the key points of these strategies, which are a combination of graph-partitioning and the bisection of blocks, which improves the expected quality of the graph-partitioning results.

\subsubsection{Graph-partitioning}

The blocks are distributed among the processors by an approximate graphpartitioning method in order to achieve a balanced distribution of work while also minimizing the amount of communicated data. If two adjacent blocks are handled by the same process, obviously no communication is needed.

In the following each block $B_{i}$ is represented by a vertex $v_{i} \in V$ in the graph $G=(V, E)$. The edges $e_{i j}=v_{i} v_{j} \in E$ in the graph $G$ indicate whether two blocks $B_{i}$ and $B_{j}$ need to communicate data with each other, i.e. ghost cells of one block are interior cells of the other block. The vertex weight $\omega_{i}=\omega\left(v_{i}\right)$ of a vertex $v_{i}$ is the computational load of its corresponding block $B_{i}$, it is determined by timing the computation on a blocks using omp_get_wtime ().

The edge weights $c_{i j}=c\left(e_{i j}\right)$ are chosen according to the number of common grid points between blocks $B_{i}$ and $B_{j}$. The computational load on process $k$ is denoted by $W_{k}=\sum_{i \in I_{k}} \omega_{i}$, with the index set $I_{k}$ indicating the Blocks on process $k$. The average load per process for $p$ process is defined as

$$
\tilde{W}=\frac{W_{\text {Total }}}{p}
$$

with $W_{\text {Total }}=\sum \omega_{i}$ being the total workload.

The goal is to divide the graph into $p$ partitions $P_{i} \subset V$, such that the edge-cut is minimized and the maximum load

$$
W_{\max }:=\max _{1 \leq i \leq p} \sum_{v \in P_{i}} \omega(v)<(1+\gamma) \tilde{W}
$$


is bounded. The graph-partitioning problem is NP-complete (see [46]). In order to work independently from graph-partitioning tools such as Zoltan [47] or METIS/ParMETIS [48] OpenPhase provides a simple graph-partitioner, that computes an approximate solution using the greedy algorithm shown in algorithm 2. Here $N(v)$ denotes the set of vertices adjacent to the vertex $v$.

As computing the partitions and exchanging the blocks can be quite costly and the

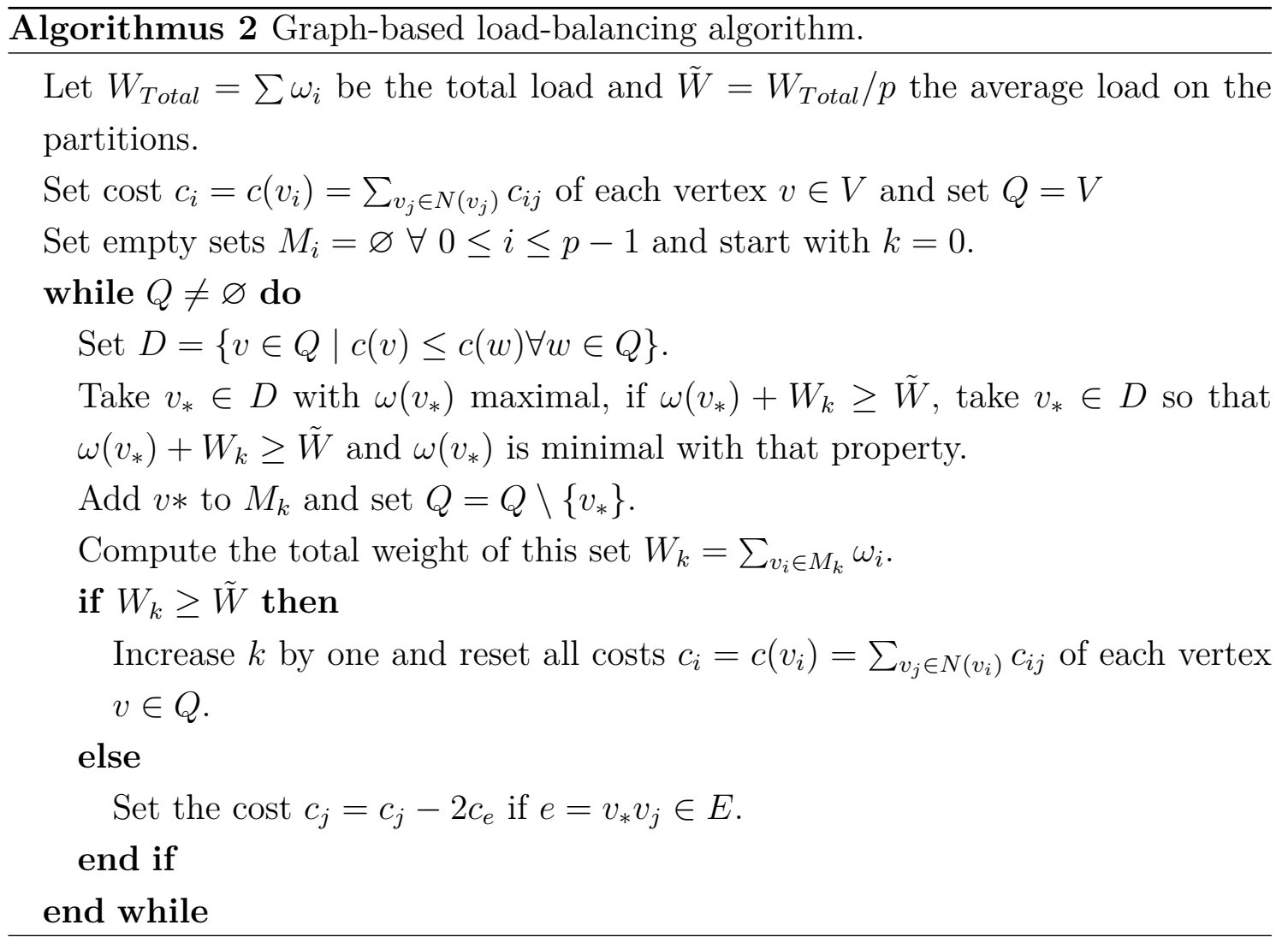

load should only change slowly over time, it is neither necessary nor beneficial to compute new block distributions too frequently. It is more efficient to measure the load and its imbalance in every time step and to compute a new block distribution if

$$
W_{k}>(1+\gamma) \tilde{W}
$$

for any block $k$ and a threshold parameter $\gamma>0$.

Proposition 5.2.1. Let $W_{\text {opt }}$ be the maximum load among partitions achieved by an optimal graph partitioning. 
Algorithm 2 fulfills

$$
W_{i} \leq 2 W_{\text {opt }} \forall i \in I
$$

Proof. Let $\omega_{\max }$ be the maximum load among the blocks. It is obvious, that $\omega_{\max } \leq$ $W_{\text {opt }}$, otherwise the partition containing the block with load $\omega_{\max }$ would have a higher load $W \geq W_{\text {opt }}$ contradicting the definition of $W_{\text {opt }}$. As the maximum load $W_{\text {opt }}$ in an optimal graph partitioning can not be smaller than the average $\tilde{W}$, we also have $W_{o p t} \geq \tilde{W}$.

Let $W_{k}^{n}<\tilde{W}$ be the load on the active partition $k$, if a vertex $v$ is added to the partition $k$, there are two possibilities:

- $W_{k}^{n+1}=W_{k}^{n}+\omega(v)<\tilde{W}$, then further vertices can be added to partition $k$.

- $W_{k}^{n+1}=W_{k}^{n}+\omega(v) \geq \tilde{W}$, then no further vertices will be added to partition $k$.

If $v$ is the last vertex to be added to partition $k$, its load will be

$$
W_{k}=W_{k}^{n+1}=W_{k}^{n}+\omega(v)<\tilde{W}+\omega_{\max } \leq 2 W_{\text {opt }} .
$$

Thus we obtain the desired result.

Remark 5.2.2. In fact, we can give an upper bound of the maximum load as

$$
W_{\max }<\tilde{W}+\omega_{\max }
$$

Thus in the worst case the relative overhead due to load-imbalances is bounded by $\omega_{\max } / \tilde{W}$ as

$$
\frac{W_{\max }}{\tilde{W}}<1+\frac{\omega_{\max }}{\tilde{W}}
$$

In addition to graph-partitioner presented in [1] two different post-optimization steps are used to improve the results of the graph-partitioner. In the first one a random vertex $v_{1} \in P_{1}$ and a random partition $P_{2}$ are chosen. The vertex is moved to this partition either if it improves the does the load-balance between partitions $P_{1}$ and $P_{2}$, i.e.

$$
W_{P_{2}}+\omega\left(v_{1}\right)<W_{P_{1}}
$$


and the edge cut is not increased, i.e.

$$
\sum_{v_{j} \in N\left(v_{1}\right) \backslash P_{2}} c_{1 j}-2 \sum_{v_{j} \in N\left(v_{1}\right) \cap P_{2}} c_{1 j} \leq \sum_{v_{j} \in N\left(v_{1}\right) \backslash P_{1}} c_{1 j}-2 \sum_{v_{j} \in N\left(v_{1}\right) \cap P_{1}} c_{1 j}
$$

or if the edge cut is decreased and maximum load $W_{\max }$ is not surpassed, i.e.

$$
W_{P_{2}}+\omega\left(v_{1}\right) \leq W_{\max }
$$

The second post-optimization option is switching the partitions of two random vertices $v_{1} \in P_{1}$ and $v_{2} \in P_{2}$ with $P_{1} \neq P_{2}$ either if the switch improves the balance between these two partitions

$$
\max \left(W_{P_{1}}-\omega\left(v_{1}\right)+\omega\left(v_{2}\right), W_{P_{2}}-\omega\left(v_{2}\right)+\omega\left(v_{1}\right)\right)<\max \left(W_{P_{1}}, W_{P_{2}}\right)
$$

without increasing the edge-cut

$$
\begin{aligned}
\sum_{v_{j} \in N\left(v_{1}\right) \backslash P_{2}} c_{1 j} & -2 \sum_{v_{j} \in N\left(v_{1}\right) \cap P_{2}} c_{1 j}+2 \chi_{N\left(v_{1}\right)}\left(v_{2}\right) c_{12} \\
+\sum_{v_{j} \in N\left(v_{2}\right) \backslash P_{1}} c_{2 j} & -2 \sum_{v_{j} \in N\left(v_{2}\right) \cap P_{1}} c_{2 j}+2 \chi_{N\left(v_{2}\right)}\left(v_{1}\right) c_{21} \\
\leq & \sum_{v_{j} \in N\left(v_{1}\right) \backslash P_{1}} c_{1 j}+\sum_{v_{j} \in N\left(v_{2}\right) \backslash P_{2}} c_{2 j},
\end{aligned}
$$

or if the switching decreases the edge-cut and the new maximum load doe not exceed the old maximum load $W_{\max }$

$$
\max \left(W_{P_{1}}-\omega\left(v_{1}\right)+\omega\left(v_{2}\right), W_{P_{2}}-\omega\left(v_{2}\right)+\omega\left(v_{1}\right)\right) \leq W_{\max } .
$$

These two post-optimization steps are alternated in a loop that terminates after the time the greedy graph-partitioner used to create the initial partition.

Remark 5.2.3. For a small number of vertices, i.e. less than 50000, the performance of the greedy graph-partitioner is sufficient. For larger graphs multilevel graphpartitioners like METIS are much more efficient.

\subsubsection{Bisection}

Corollary 5.2.2 implies that if we reduce the cost $\omega_{\max }$ of the most costly block, we can reduce the upper bound of the load-imbalance. A reduction of the maximum 

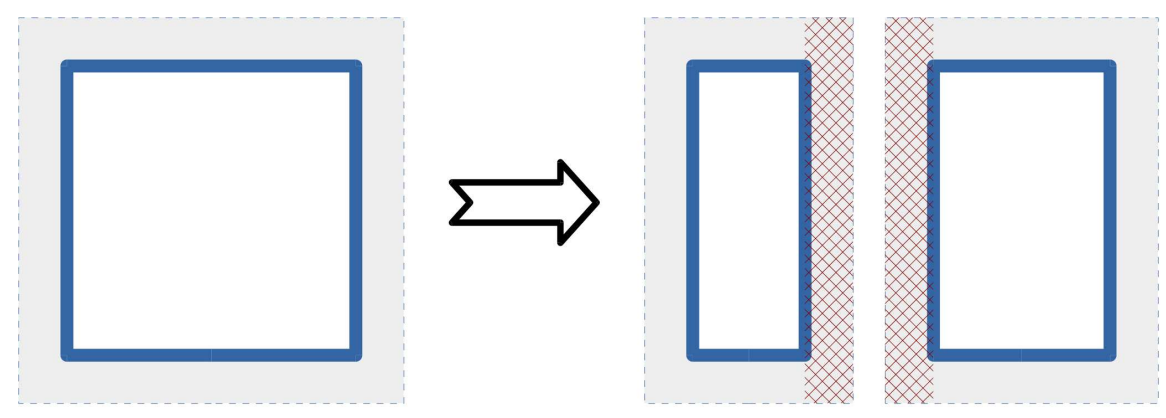

$\omega$

$\omega_{1}+\omega_{2}=\omega+\omega_{c u t}$

Figure 5.5: Splitting a block in two parts introduces additional work in the wide halo.

load $\omega_{\max }$ can be achieved by a bisection of this blocks into two smaller blocks. This, however, will introduce additional communications and duplicated computations of the overlap between these two blocks. This makes the bisection of the block with the maximum load $\omega_{\max }$ not feasible if $\omega_{\max }$ is already comparatively small, so that the expected performance benefit of a better load-balance is nullified by the additional costs.

We calculate an optimal maximum load $\omega_{\text {opt }}$, such that the blocks with a load higher than $\omega_{\text {opt }}$ are split into two blocks. As described in [1], we have to compute a minimum of $g(\omega)$, defined by

$$
g(\omega)=\sum_{i \in I} \frac{\omega_{\text {cut }}\left(B_{i}\right)}{p} \max \left[0,\left\lceil\log _{2}\left(\frac{\omega\left(B_{i}\right)}{\omega}\right)\right\rceil\right]+\omega
$$

in order to find the optimal maximum load $\omega_{\text {opt }}$ of blocks.

The function $g$ is obtained in the following way: Let $B$ be a block with computational load $\omega(B)$. If block $B$ is split in two blocks $B_{1}$ and $B_{2}$ as in figure 5.5, these will have loads $\omega\left(B_{1}\right)$ and $\omega\left(B_{2}\right)$. Generally the amount of work will increase as

$$
\omega_{\text {cut }}(B)=\omega\left(B_{1}\right)+\omega\left(B_{2}\right)-\omega(B),
$$

which is the overhead due to duplicated computations in the wide halo of the blocks. If we cut the block $B_{\max }$ with the highest load, we get

$$
W_{\max }^{(1)}<\tilde{W}^{(1)}+\omega_{\max }^{(1)}
$$


with the new highest load $\omega_{\max }^{(1)}$ among the blocks after the cut and the new average load

$$
\tilde{W}^{(1)}=\tilde{W}+\frac{\omega_{c u t}\left(B_{\max }\right)}{p},
$$

which includes the old average $\tilde{W}$ and newly introduced work $\omega_{c u t}\left(B_{\max }\right)$. If we repeatedly cut different blocks $B_{i}$ until $\omega\left(B_{i}\right)<\omega_{m a x}^{(n)}$ for all blocks $B_{i}$, we get

$$
W_{\max }^{(n)}<\tilde{W}+\sum_{i \in I_{\text {cut }}^{(n)}} \frac{\omega_{c u t}\left(B_{i}\right)}{p}+\omega_{\text {max }}^{(n)} .
$$

We have to consider blocks that are split in multiple stages. If a block $B$ is split in blocks $B_{1}$ and $B_{2}$, we make the assumption $\omega_{\text {cut }}(B) \approx \omega_{\text {cut }}\left(B_{1}\right)+\omega_{\text {cut }}\left(B_{2}\right)$. The number of times a block is recursively cut is given by $\left\lceil\log _{2}\left(\frac{\omega\left(B_{i}\right)}{\omega_{\max }^{(n)}}\right)\right\rceil$. In order to find the optimal maximum size $\omega_{\text {opt }}$ of blocks we have to find a minimum of $g(\omega)$, defined by

$$
g(\omega)=\sum_{i \in I} \frac{\omega_{\text {cut }}\left(B_{i}\right)}{p} \max \left[0,\left\lceil\log _{2}\left(\frac{\omega\left(B_{i}\right)}{\omega}\right)\right\rceil\right]+\omega
$$

This function is monotonically increasing in each interval divided by

$$
\frac{\omega\left(B_{1}\right)}{1}, \frac{\omega\left(B_{1}\right)}{2}, \frac{\omega\left(B_{1}\right)}{4}, \ldots, \frac{\omega\left(B_{1}\right)}{2^{n}}, \frac{\omega\left(B_{2}\right)}{1}, \frac{\omega\left(B_{2}\right)}{2}, \frac{\omega\left(B_{2}\right)}{4}, \ldots,
$$

and for practical purposes it is usually enough to subdivide blocks up to three or four times. If blocks are divided further they will get too small compared to the size of the wide halo.

We find the optimal maximum size $\omega_{\text {opt }}$ by testing at these points for the reasonable choice of $n=4$. This only sets a limit on how many times a block is split in one load balancing step. If it is possible and necessary to split a block further, splitting will occur in additional load balancing steps.

Conversely, in order to save redundant computations on the halo as well as communications between blocks, two blocks $B_{i}$ and $B_{j}$ that are located on the same process and are able to form a cuboid are combined if

$$
\omega\left(B_{i}\right)+\omega\left(B_{j}\right)<\omega_{\text {opt }} .
$$




\subsubsection{Estimation of Computational Load}

In section 5.2.2 and especially in equation 5.17 we make use of the computational loads on blocks, while $\omega(B)$ for an existing blocks $B$ is known, $\omega_{\text {cut }}(B), \omega\left(B_{1}\right)$ and $\omega\left(B_{2}\right)$ are not. The load on a blocks $B$ can be assumed to be sum of the loads $\omega\left(x_{h}\right)$ of individual grid points $x_{h} \in B$

$$
\omega(B)=\sum_{x_{h} \in B} \omega\left(x_{h}\right),
$$

While the determination of the load $\omega\left(x_{h}\right)$ directly is possible it is not feasible as timing each individual grid point would increase the computational load severely. Considering the implementation details given in section 2.1 and especially 2.5 we notice that the amount of computational work in a grid point $x_{h}$ depends on the number $n\left(x_{h}\right)$ of locally active phase fields. On the one hand we have operations such as the settings of tags as described in section 2.3, that are independent of the number $n\left(x_{h}\right)$ of active phase fields and therefore scale with $n\left(x_{h}\right)^{0}$. On the other hand we have operations that are heavily dependent on the number $n\left(x_{h}\right)$ of locally active phase fields and scale with $n\left(x_{h}\right)^{3}$, such as the computation of the interface energy $\dot{\psi}_{\alpha \beta}^{\mathrm{GB}}$ in equation 2.9. Therefore we use the approximation

$$
\omega\left(x_{h}, \kappa\right) \approx \tilde{\omega}\left(x_{h}\right):=\sum_{i=0}^{m} \kappa_{i} n\left(x_{h}\right)^{i},
$$

with the number of non-zero phase fields $n\left(x_{h}\right)$ at point $\left(x_{h}\right)$ to determine the load indirectly. This accounts for operations that done a constant number of times per grid point, operations that are executed proportionally to the number of active phase fields in a grid point and to the number of combinations of two or three active phase-fields. With the constrained Broyden-Fletcher-Goldfarb-Shanno algorithm as implemented in dlib [49] we find the minimum of

$$
g(\kappa):=\sum_{B \in S}\left[\omega(B)-\sum_{x_{h} \in B} \tilde{\omega}\left(x_{h}, \kappa\right)\right]^{2}
$$

with $\kappa_{i} \geq 0$ for $0 \leq i \leq m$ and a set $S$ of finished computations on blocks. Figures 5.6 and 5.7 show the correlation of the estimate

$$
\tilde{\omega}(B)=\sum_{x_{h} \in B} \tilde{\omega}\left(x_{h}\right)
$$




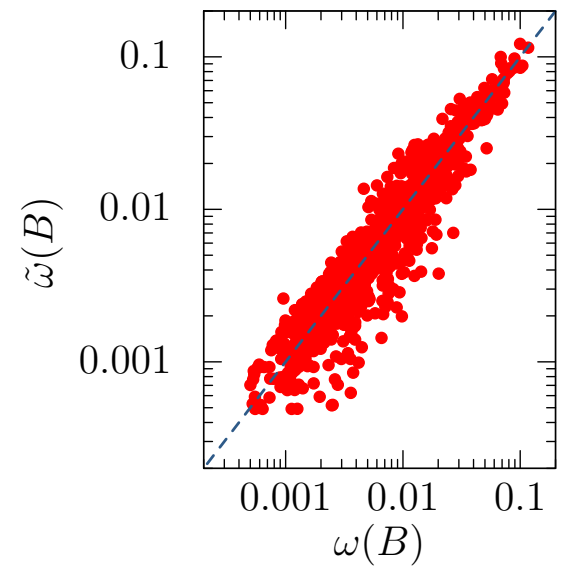

Figure 5.6: The correlation between $\omega(B)$ and the estimate $\tilde{\omega}(B)$ for the grain growth scaling test with a Gaussian particle distribution in section 6.2.2. The correlation coefficient is $r=0.95307$.

to the load $\omega(B)$ on block $B$ for the grain growth scaling test with a Gaussian particle distribution in section 6.2.2 as well as the $\mathrm{Mg}-\mathrm{Al}$ alloy solidification scaling test with a Gaussian particle distribution in section 6.5.2.

Remark 5.2.4. The computational load of stencil operations can also depend on the number of locally active phase fields within the stencil region, therefore the spatial arrangement of phase fields can influence the computational load as well. For simplicity this is not considered in estimation 5.25.

\subsubsection{Determination of the Cutting Plane}

Using estimate 5.25 cutting planes for the bisection are selected that are aligned to the grid. For each of the three normal directions $d=x, y$ or $z$ a cutting plane is determined for which the difference $C_{d}:=\left\|\tilde{\omega}\left(B_{1}\right)_{d}-\tilde{\omega}\left(B_{2}\right)_{d}\right\|$ of the loads of the two resulting blocks is minimal. The cutting plane with normal $d$ that has the minimal difference $C_{d}$ is chosen and the block is split along this plane.

Remark 5.2.5. The number of ghost cell layers poses a constraint on the minimum size of the blocks as we do not allow communication with blocks that are not direct neighbors. 


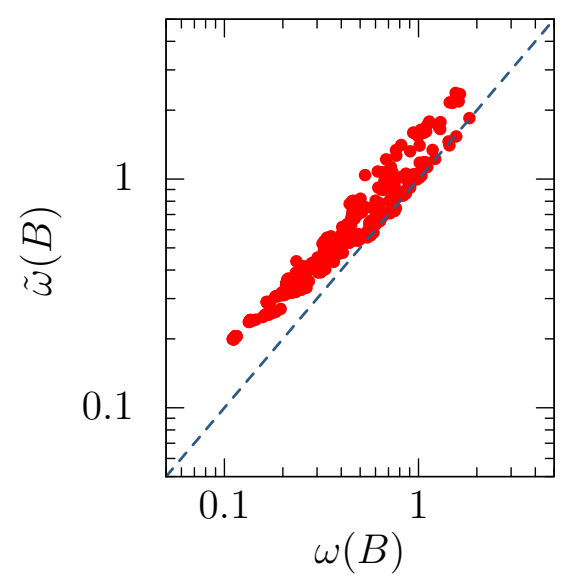

Figure 5.7: The correlation between $\omega(B)$ and the estimate $\tilde{\omega}(B)$ for the $\mathrm{Mg}-\mathrm{Al}$ alloy solidification scaling test with a Gaussian particle distribution in section 6.2.2. The correlation coefficient is $r=0.93805$.

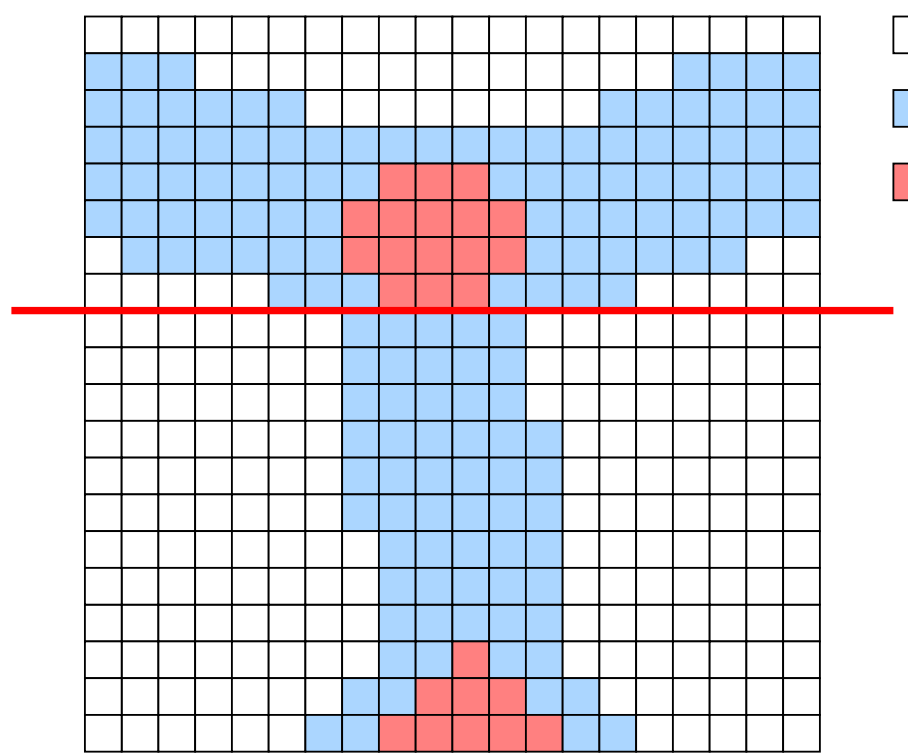

One Phase

Two Phases

Three Phases

Figure 5.8: The bisection does not necessary split a block into two equally sized halves. The block is rather split into two blocks of approximately equal computational load. Here the parameters $\kappa_{0}=1.8 \cdot 10^{-6} \mathrm{~s}, \kappa_{1}=3.0 \cdot 10^{-6} \mathrm{~s}$ and $\kappa_{2}=5.5 \cdot 10^{-6} \mathrm{~s}$ are used as defined in section 5.2.3. Those values are close to those obtained during the benchmark for the $\mathrm{MgAl}$-solidification benchmark in section 6.3 on Jureca. 
Remark 5.2.6. Using the above estimation the overhead by bisection only considers additional computations, but not an added cost for communication. Therefore it makes sense to modify equation 5.21 to

$$
g(\omega)=\theta \sum_{i \in I} \frac{\omega_{\text {cut }}\left(B_{i}\right)}{p} \max \left[0,\left\lceil\log _{2}\left(\frac{\omega\left(B_{i}\right)}{\omega}\right)\right\rceil\right]+\omega
$$

with a parameter $\theta \geq 1$.

Remark 5.2.7. If we set $(1+\gamma) \tilde{W}=\tilde{W}+\omega_{\text {opt }}$ the threshold parameter $\gamma$ in inequality 5.3 becomes

$$
\gamma:=\frac{\omega_{o p t}}{\tilde{W}}
$$

which means that we compute a new block distribution if the load $W_{k}$ on a process $k$ is larger than the previous $W_{\max }$ due to

$$
W_{k} \geq(1+\gamma) \tilde{W}=\tilde{W}+\omega_{o p t}>W_{\max }
$$

\subsubsection{Data Migration}

After the assignment of blocks to processes by the graph-partitioner some of the blocks may need to be moved to another process. The way of sending the data is the same as in section 5.1.2, with the only difference that the entire block is send to another process and not just the overlapping grid points of blocks. As the amount of data that needs to be migrated can be extensive, one needs to account for enough memory on each process to simultaneously hold the data that it retains, the data that it needs to send to other processes and the data that it receives data from other processes. As data migration in this way can be very costly, the system should not be rebalanced in every step. For systems that in which the load-distribution does not change significantly over time, the initial cost for load-balancing and data migration can be neglected. For dynamic systems however the cost of updating the domain decomposition and the data migration can be a significant contributing factor for the wall time of a program, which will be addressed in section 5.2.7. 


\subsubsection{The Full Balancing Algorithm}

In the beginning of the simulation the user has control over the initial domain decomposition. He can set the size of blocks and the number of blocks in each direction. At the start all blocks have the same size and are automatically assigned to processes so that the number of blocks per process differs at most by one, computational load is not considered at this stage. By calling the balancing method the following steps are executed as also depicted in figure 5.9:

1. Determination of the parameters $\kappa_{i}$ as defined in section 5.2.3.

2. Determination of the cutting planes for all blocks $B$ as described in section 5.2.4 and computing the cost of cutting $\tilde{\omega}_{c u t}(B)$.

3. Minimization of function 5.21 and obtaining the optimal maximum size $\omega_{\text {opt }}$.

4. If $W_{k}>\tilde{W}+\omega_{\text {opt }}$, splitting of all Blocks $B$ with load $\omega(B)>\omega_{\text {opt }}$ along the precomputed cutting plane.

5. Merging of blocks $B_{i}$ and $B_{j}$ if they are located on the same process, form a cuboid and fulfill $\omega\left(B_{i}\right)+\omega\left(B_{j}\right)<\omega_{\text {opt }}$.

6. If $W_{k}>\tilde{W}+\omega_{\text {opt }}$, graph-partitioning in order to reassign blocks to processes. Remark 5.2.8. In order to reduce the overhead created by the wide halo it is preferred to design blocks as large as possible. Therefore it can be beneficial to use hybridparallelism as depicted in figure 5.9, combining MPI and OpenMP. This way the number of processes $p$ in equation 5.28 gets smaller and blocks remain larger, while still maintaining balance between the processes. Using dynamic scheduling within OpenMP allows easy load-balancing between threads associated to one MPI-process.

\subsubsection{Dynamic Load-balancing}

During the course of a simulation the distribution of the computational load can change significantly. In order to keep the computational load on the processes ba- 

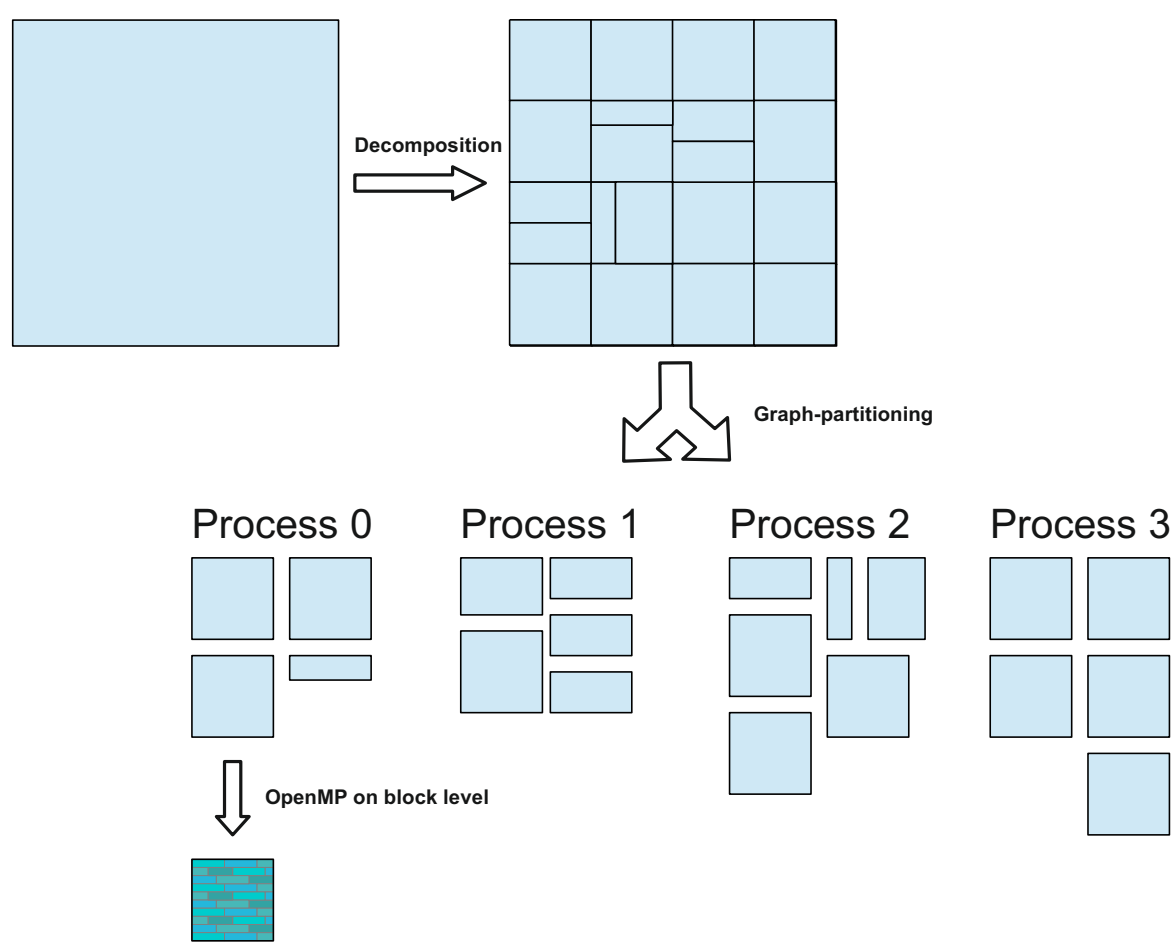

Figure 5.9: The computational domain is sub-divided into smaller blocks, that are assigned to MPI-processes by a graph-partitioner. OpenMP provides further parallelism on each block.

lanced it is required to dynamically compute new domain decompositions during the simulation in order to account for the changing load conditions. As computing a new domain-decomposition can be quite costly itself as it contains the bisectioning and merging of blocks as well as data migration, we do not want to compute new domain-decompositions unless we assume it is beneficial.

If we have computed a new domain decomposition in time step $k$ it makes sense to update the next domain decomposition in step $k+n$ for which the average wall time per time step

$$
\tilde{T}_{k}(n):=\frac{1}{n}\left(T_{k+n}^{L B}+\sum_{i=k}^{k+n} T_{i}^{k}\right)
$$

between load-balancing steps is minimal; $T_{i}^{k}$ denotes the wall time for time step $i$ after load-balancing in time step $k$ and $T_{k+n}^{L B}$ denotes the wall time for load-balancing in time step $k+n$. Thus we have to find $n \in \mathbb{N}$ that minimizes the function $\tilde{T}_{k}(n)$. As the number of block bisections and merging steps as well as the amount of 
communication can change, the wall times of the balancing steps are generally not constant and we do not know the wall time $T_{k+n}^{L B}$ for the balancing algorithm in time step $k+n$ a priori. Therefore we use the exponential moving average $\tilde{T}_{k+n}^{L B}$

$$
\tilde{T}_{k+n}^{L B}:=\lambda T_{k}^{L B}+(1-\lambda) \tilde{T}_{k}^{L B},
$$

with the last wall time for load-balancing $T_{k}^{L B}$ in time step $k$ and the prior average $\tilde{T}_{k}^{L B}$. Now we search for the first time step $k+n$ that is a local minimum of

$$
\tilde{T}_{k}(n):=\frac{1}{n}\left(\tilde{T}_{k+n}^{L B}+\sum_{i=k}^{k+n} T_{i}^{k}\right)
$$

that is the first time step with

$$
\frac{1}{n}\left(\tilde{T}_{k+n}^{L B}+\sum_{i=k}^{k+n} T_{i}^{k}\right)<\frac{1}{n+1}\left(\tilde{T}_{k+n}^{L B}+\sum_{i=k}^{k+n+1} T_{i}^{k}\right) .
$$

Under the assumption that load-balancing creates a proper domain decomposition, that is at least as good as the domain decomposition obtained the last time the load-balancing was carried out, it would have made sense to re-balance the system after time step $k+n$. However, as the time step $k+n+1$ has already finished in order to obtain the time $T_{k+n+1}^{k}$, re-balancing is carried out after time step $k+n+1$. If the wall time per time step is not increasing no $n \in \mathbb{N}$ exists that fulfills inequality 5.34. However, there can be situations in which the wall time per time step is not increasing, but the load distribution changes so that a better load-balance becomes possible. Therefore it is useful to use a secondary criterion for load-balancing, such as periodically calling the balancing algorithm of section 5.2 .6 every $m$ steps.

\subsection{Parallel I/O}

As large scale simulations also need scalable I/O-operations. High-Performance parallel cluster use parallel filesystems such as PanFS [50] on Vulcan at ICAMS or the IBM General Parallel File System (GPFS) [51] on JURECA at Forschungszentrum Jülich to allow concurrent data access from multiple compute nodes in a unified address space. In order to take advantage of a parallel file system all processes 
should participate in I/O-operations, in which each process writes its own data simultaneously. This can be achieved by each process writing its data to its own file. This approach is easy to implement, but can reduce the usability for postprocessing or visualization as the individual data files need to be combined correctly. Additionally using many files can reduce the performance of the parallel file system, as a significant overhead is introduced. Additionally opening a large number of smalls file can also create performance issues for a parallel file system. Generally the best performance is achieved using collective I/O operations with large chunk sizes [52].

OpenPhase uses the XML file format of VTK for data output [53]. While VTK provides a parallel file type, OpenPhase only uses the serial VTK file type, but writes the data to it in parallel. The parallel VTK file type creates single files for each segment and an additional file for the storage of meta data that allows easy processing using tools such as Paraview or Visit.

In OpenPhase the output of VTK data is achieved in the following way: Each process creates a write buffer of type std::stringstream that contains the data on each block. Each block is assembled in a piece

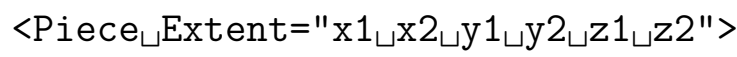

$<$ PointData $>$. . </PointData $>$

$<$ CellData $>$. . $</$ CellData $>$

$<$ Points $>$. . </Points $>$

$</$ Piece $>$

with an extent given by its interior global coordinates. Each process concatenates the piecewise information of each of its block in the buffer. Using MPI_Allgather each process sends the size of its buffer to all other processes. The process with rank 0 creates the XML file header

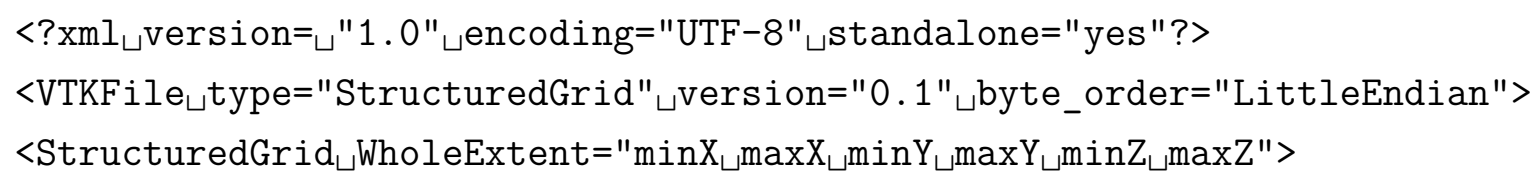

as well as the tail 


\section{Parallelization}

$</$ StructuredGrid $>$

$</$ VTKFile $>$

and broadcasts the size of the header to the other processes. Using the information of the header size as well as all buffer sizes each process can compute the offset at which it needs write its own data which is conducted by the collective call

MPI_File_write_at_all 


\section{Applications}

The following chapter focuses on different applications for the simulation of material evolution using the multiphase field method and reiterates the results presented in [3]. The applications of normal grain growth in section 6.1 and solidification of $\mathrm{Mg}-\mathrm{Al}$ alloys in section 6.3 are covered and large scale simulations that were previously impossible without the increased resources of the distributed-memory parallelization are presented. These applications serve as benchmarks for the scaling properties of the parallelization presented in chapter 5. Afterwards the Mg-Al alloy solidification simulations are expanded by a coupling with the Lattice-Boltzmann method in order to observe the effect of solidification on the melt flow.

\subsection{Normal Grain Growth}

The micro structure of structural materials is often sub-divided into a large number of atom clusters, called grains, with different properties such as the composition or the crystallographic orientation. Grain growth refers to the growth of larger grains at the expense of smaller grains in order to reduce internal energy by reduction of the grain boundary area. The multiphase field method is a promising tool for the simulation of grain growth with a large number of different phase fields $[54,5,6]$. In practice, however, the large number of grains needed for statistically relevant simulations creates significant requirements on both memory and computational power. Early phase-field simulations of grain growth suffered from poor statistics due to limited box size $[55,56]$. Even though the simulation capacity gradually grew, the number of possible independent phase-fields was still limiting. A solution 
for this problem was to introduce multiple instances of a finite number of different grains, which improved the statistical accuracy of the results $[57,58]$. However it is problematic that grains with the same indexes may coalesce during the simulation. This might explain why only recently the correlation between self-similarity and topological characteristics of normal grain growth was investigated [11].

In a previous study [12] an efficient storage structure combined with OpenMP parallelization was applied to investigate normal grain growth. Despite significant improvements in statistical and topological analysis, the outlook of the results points yet towards larger and more efficient simulations [11]. Thus a careful implementation of massive parallelization became evident.

A simulation of grain on a computational domain consisting of $512^{3}$ grid points with 30000 grains was conducted. A narrow distribution of the grains was initialized via Voronoi tessellation[59]. Initial grains are small compared to the box size, but sufficiently large with respect to the grid spacing $\Delta x$ and interfacial width $\eta=$ $6 \Delta x$. Nevertheless, the early stages of simulation are omitted in the analysis to stay securely away from numerical artifacts. The interfacial energy and mobility have been chosen as $1 \mathrm{Jm}^{-2}$ and $10^{-14} \mathrm{~m}^{4} \mathrm{~J}^{-1} \mathrm{~s}^{-1}$ respectively, within the accepted range for metallic systems. The time step and grid spacing are $\mathrm{dt}=10^{-1} \mathrm{~s}$ and $\Delta x=10^{-7}$ m.

A second even larger simulation was conducted, which used $1024^{3}$ grid points and 240000 initial grains. One can easily notice that a simulation of such scale would never be possible without sparse storages and active parameter tracking. Still it requires significant resources and was run 512 cores on the Vulcan cluster at ICAMS, it used 32 nodes with 128 GB of memory, which was barely enough. The data was initialized by eight individual Voronoi tessellations using Voro ++ [59]. Each of them created 30000 grains in a domain of $512^{3}$ grid points. These partial configurations were combined to create the initial grain distribution for the large simulation. In figure 6.1 (a) one can see that the numbering of the grains represents this initialization.

Handling the VTK output for this simulation proofed to be problematic as both ParaView [60,61] and Visit [62] ran out of memory of the visualization nodes of 
JURECA while trying to visualize the data. These visualization nodes provide 512 GB of memory and 2 NVIDIA Tesla K40 GPUs. A downsampling of the data was required before visualization. Figure 6.1 shows the visualization of data that has been downsampled by a factor of 4 in each dimension to a size of $256^{3}$ grid points. This allowed visualization of the data on the visualization nodes of the Vulcan cluster at ICAMS, which only have a memory of 128 GB.

In the following a series of benchmarks both for performance and accuracy of the results is presented. The results of the study are compared against previous studies.

\subsubsection{Results and Discussion}

A characteristic property of any polycrystalline is its averaged grain size that is subject to the changes during grain growth. For an ideal material, like in our case, a parabolic relation

$$
\langle R\rangle^{2}=K t+R_{0}^{2}
$$

is proposed theoretically, where $K=A \mu \sigma$ and $R_{0}$ is the initial grain size. Here $\mu$ and $\sigma$ are interface mobility and energy, respectively, and parameter $A$ reflects the overall geometry of grains. The results for the growth kinetics are shown in Figure 6.2. The linear trend of the graph can be well described by eq. 6.1 revealing the parabolic kinetics of the growth. In the simulations, the value for parameter $A$ is obtained as 0.48 which is well comparable to the value 0.5 found in the previous studies using serial computations $[12,63]$. The results are presented after 2000 initial time steps, where the steady state growth is achieved. Figure 6.3 shows the snapshots of the simulation after 2000 and 10000 time steps.

Another characteristic measure of the system is the distribution of grains with respect to their size (GSD). Here also the parallel computation of our system finely recovers the GSD and the trend of its change over the entire simulation period. After 


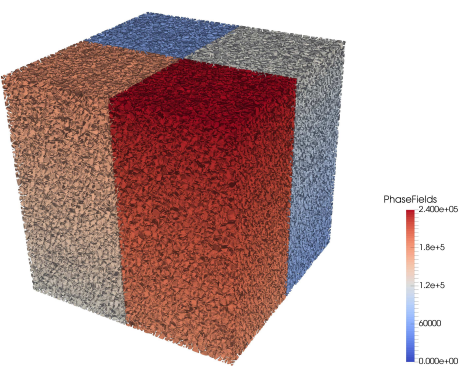

(a) Start configuration with 240000 grains

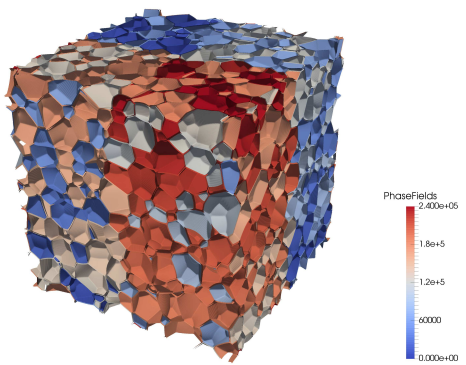

(c) After 40000 time steps

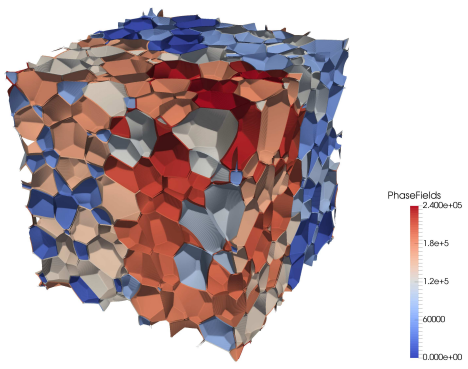

(e) After 80000 time steps

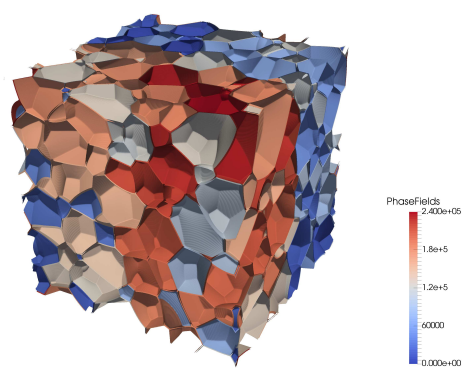

(g) After 120000 time steps

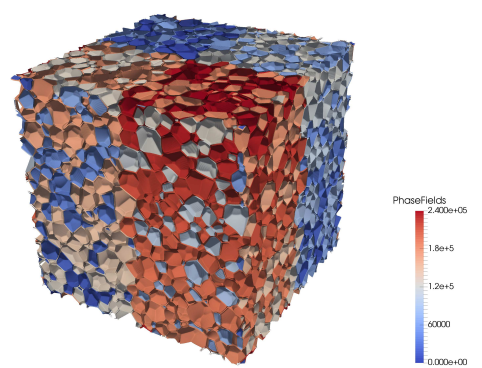

(b) After 20000 time steps

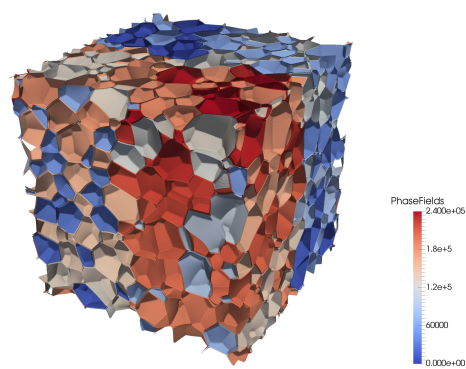

(d) After 60000 time steps

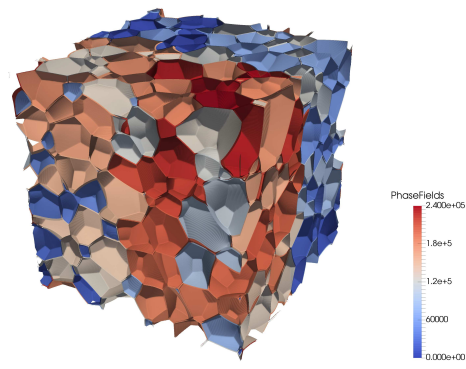

(f) After 100000 time steps

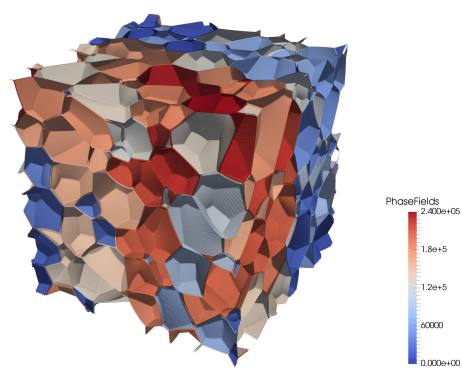

(h) After 140000 time steps

Figure 6.1: Simulation of normal grain growth on a computational domain of $1024^{3}$ grid points with 240000 initial grains. The colors represent the index of the phase field. 


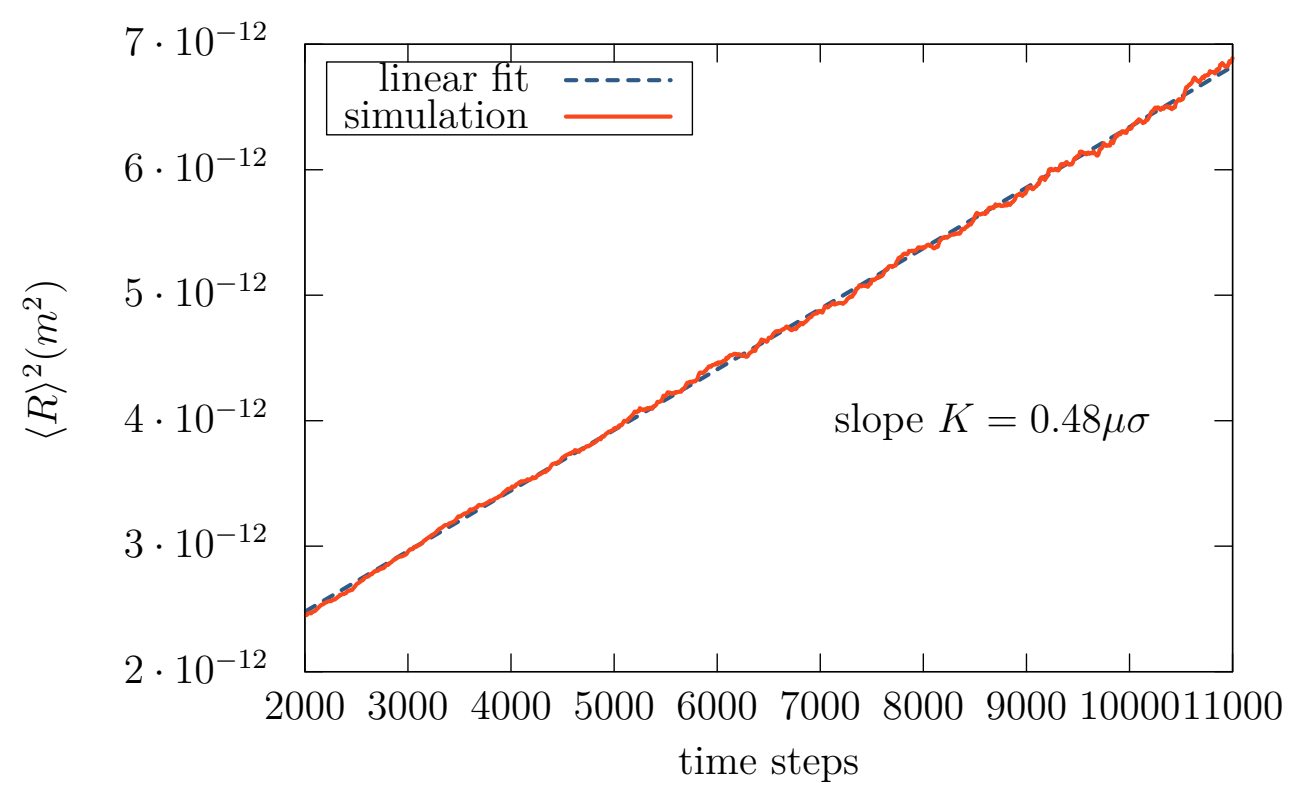

Figure 6.2: Squared average grain sizes over time.

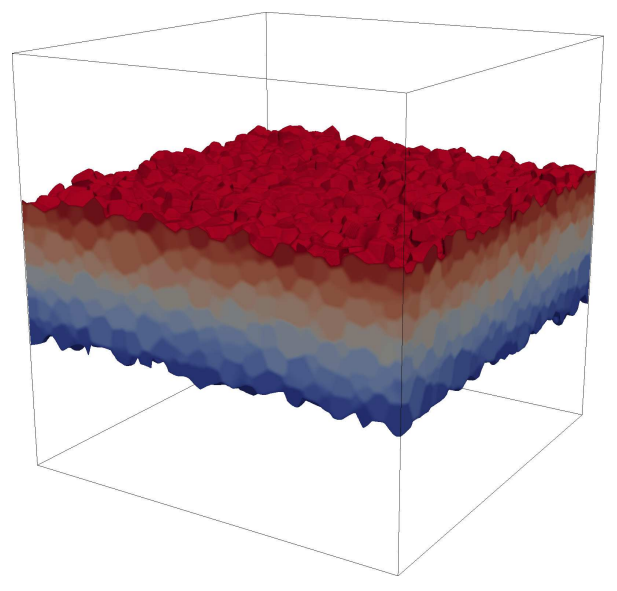

(a) Grains after 2000 time steps

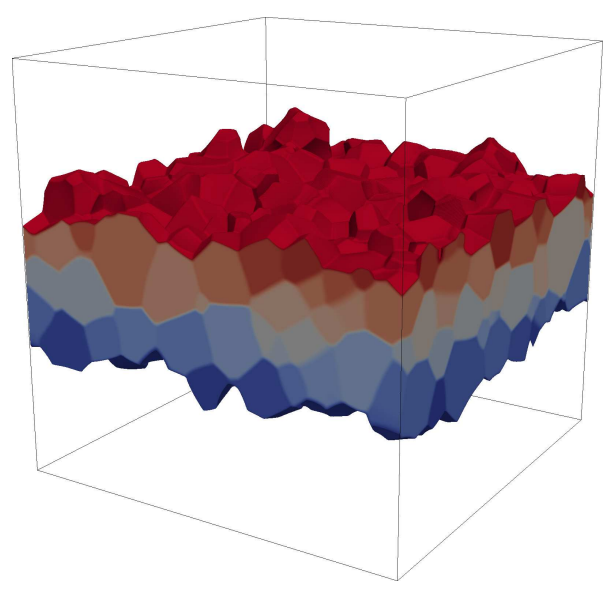

(b) Grains after 10000 time steps

Figure 6.3: Normal grain growth simulation of section 6.1.

about 2000 time steps, the size distribution matches the well-known Hillert's analytical solution [64] as seen in figure 6.4. The predicted deviation from this solution after 10000 time steps is also well recovered. 


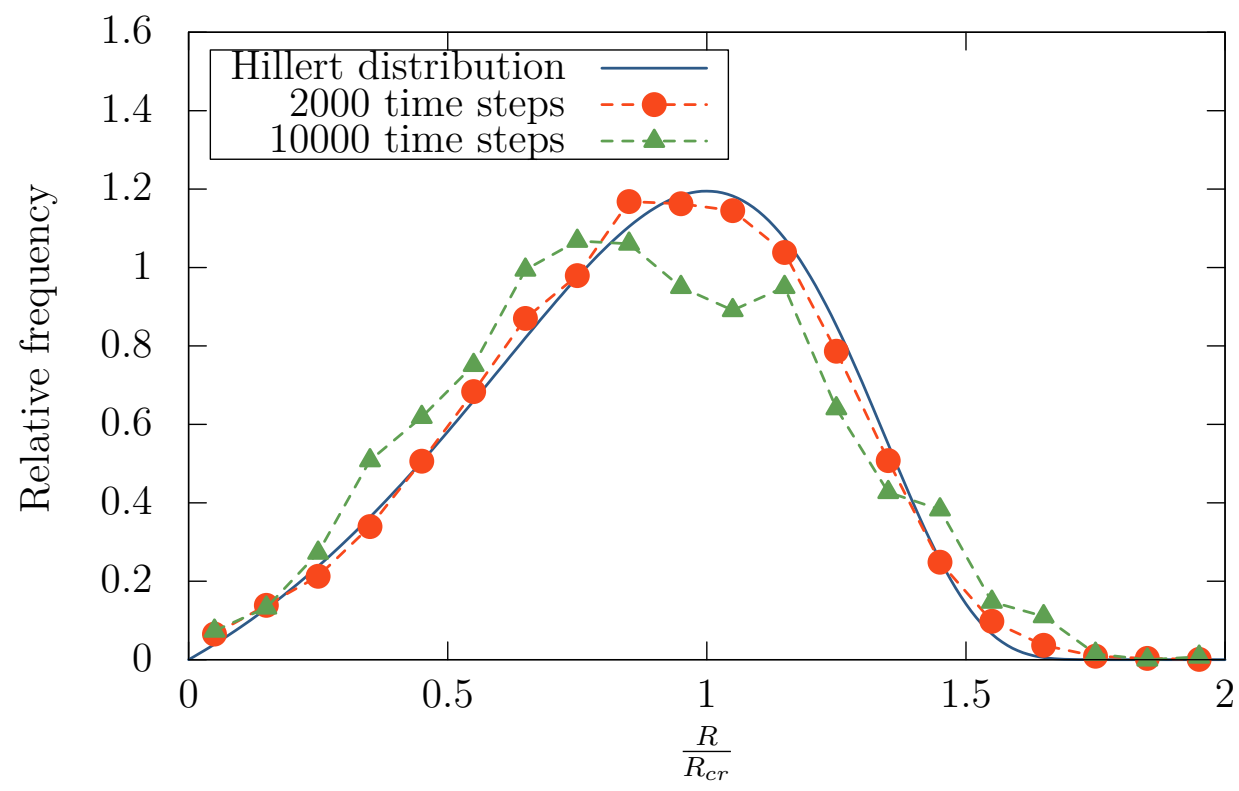

Figure 6.4: Comparison of the simulation results to the Hillert distribution [64] with $R_{c r}=$ $\frac{9}{8}\langle R\rangle$.

\subsection{Scaling Benchmark for Grain Growth Simulations}

In the following section we look at the performance in a series of benchmark test that reflect the computational demands of grain growth simulations as discussed in section 6.1. The benchmarks use exactly the same algorithms as the normal grain growth application in section 6.1, however, for simplicity the initial data consists of random distribution of particles in a matrix. This saves the work of generating a Voronoi tessellation of the domain and allows the usage of the same particle distribution in the alloy solidification benchmarks in section 6.3. Physically this simulation corresponds to precipitate coarsening in a solid matrix. Precipitates and matrix are of the same thermodynamic phase, but have different crystallographic orientations.

For the benchmark we setup the simulation using $N$ blocks with

$$
N=2 N_{\mathrm{MPI}},
$$

where $N_{\text {MPI }}$ is the number of MPI processes. Each block has a size of $64 \times 64 \times 64$ grid points in single threaded benchmarks, with two threads per process the size of 


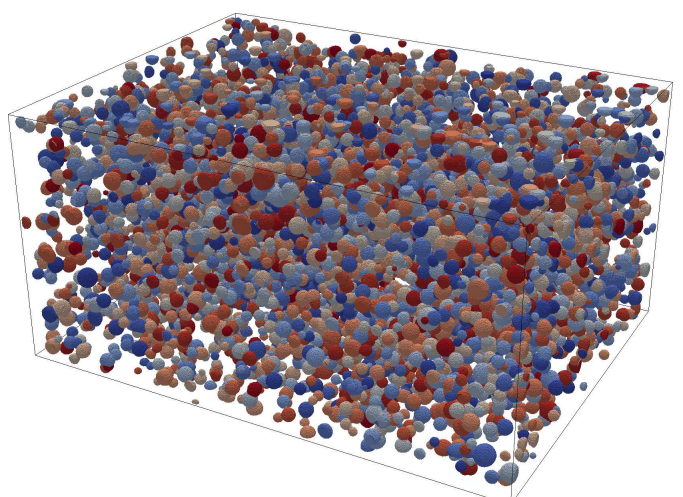

(a) Uniform particle distribution

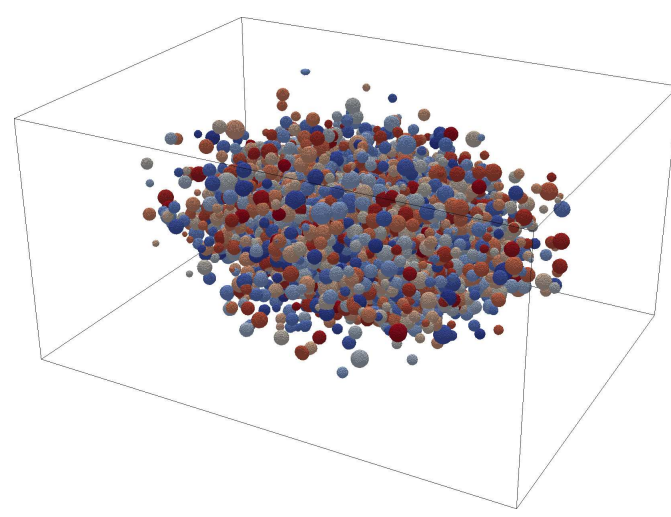

(b) Gaussian particle distribution

Figure 6.5: Initial conditions for the benchmarks in section 6.2. Both start with the same number of particles, however on the right hand side, the particles in the center of the domain form more multiple junction which increases the computational load severely, while the amount of work near the boundary of the domain is very low.

each blocks is $128 \times 64 \times 64$ and with four threads it is $128 \times 128 \times 64$. The blocks are assembled in a way so that the computational domain $\Omega$ forms a cuboid with the minimal surface area. Therefore we have 12,582,912 grid points for 24 cores and go up to 402,653,184 grid points for 768 cores.

For testing purposes spherical particles will be generated at random positions with a uniform and a Gaussian distribution of the particle positions as depicted in figure 6.5. A halo size of two layers of grid points is used, one layer is needed for the stencil operation in equation 2.25 and another layer is needed to tag grid points as interface or near-interface. In this test the load distribution does not change rapidly and once a domain decomposition with a suitable load-balance is found no further loadbalancing steps need to be performed. For the first 300 time steps the load-balancing algorithm as presented in section 5.2 .6 will be called every 10 time steps. This is done to give the algorithm enough steps to adapt the block sizes as in on balancing step each block is only allowed to be split once or merged once. The timing results are obtained from the average of the next 100 time steps, during which no further load-balancing occurs. 


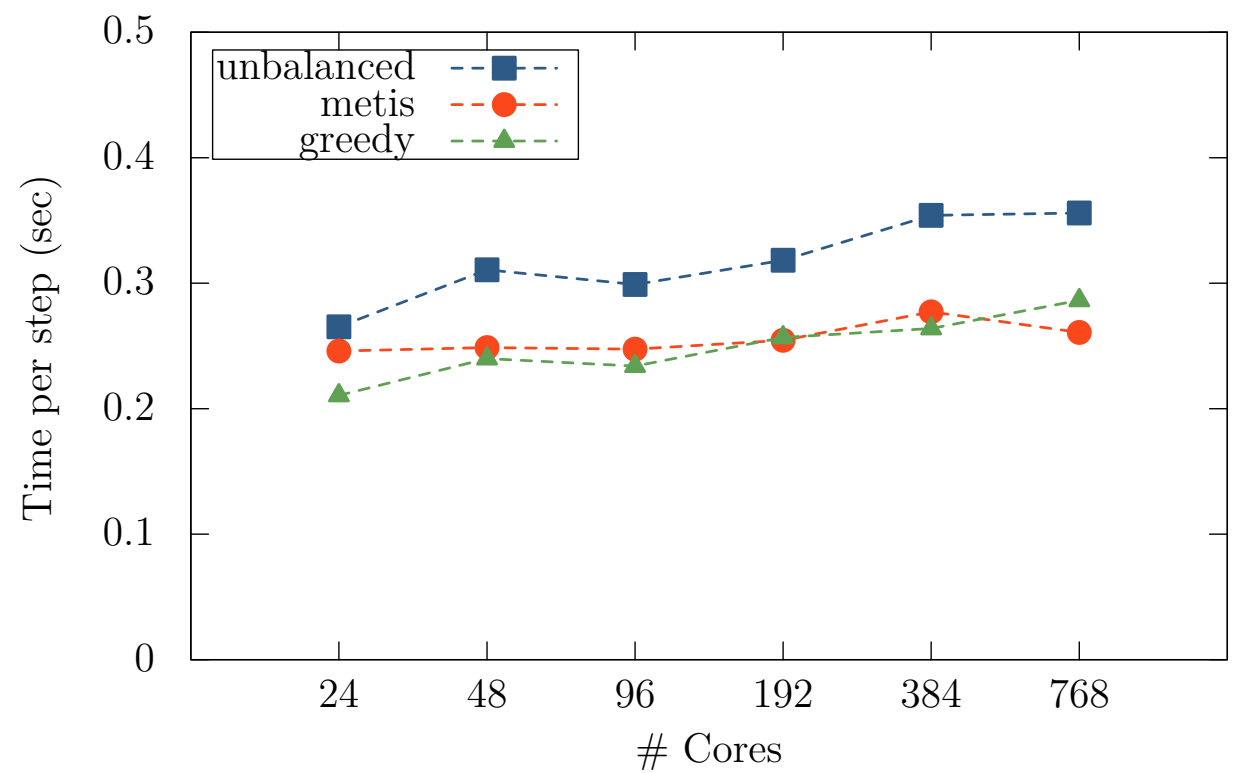

Figure 6.6: Grain growth scaling benchmark for a uniform particle distribution, see section 6.2.1. Although in this case there are no significant load-imbalances, loadbalancing using either METIS or the greedy graph-partitioner provided with OpenPhase are beneficial to the performance.

\subsubsection{Uniform Distribution of Particles}

In the domain $\Omega$ we embed $N_{p}=10 \times N_{\text {MPI }} \times N_{\text {OpenMP }}$ spherical particles at random coordinates. The particles are distributed uniformly in the domain and the particle sizes follow a normal distribution with a mean radius of $\mu_{r}=10$ and a standard deviation $\sigma_{r}=2$. This test represents a simulation without significant load-imbalances.

In this test only very minor load-imbalances occur, however, as seen in figure 6.6 load-balancing using bisection and either METIS or the greedy graph-partitioner mentioned in section 5.2.1 are beneficial to the performance. 

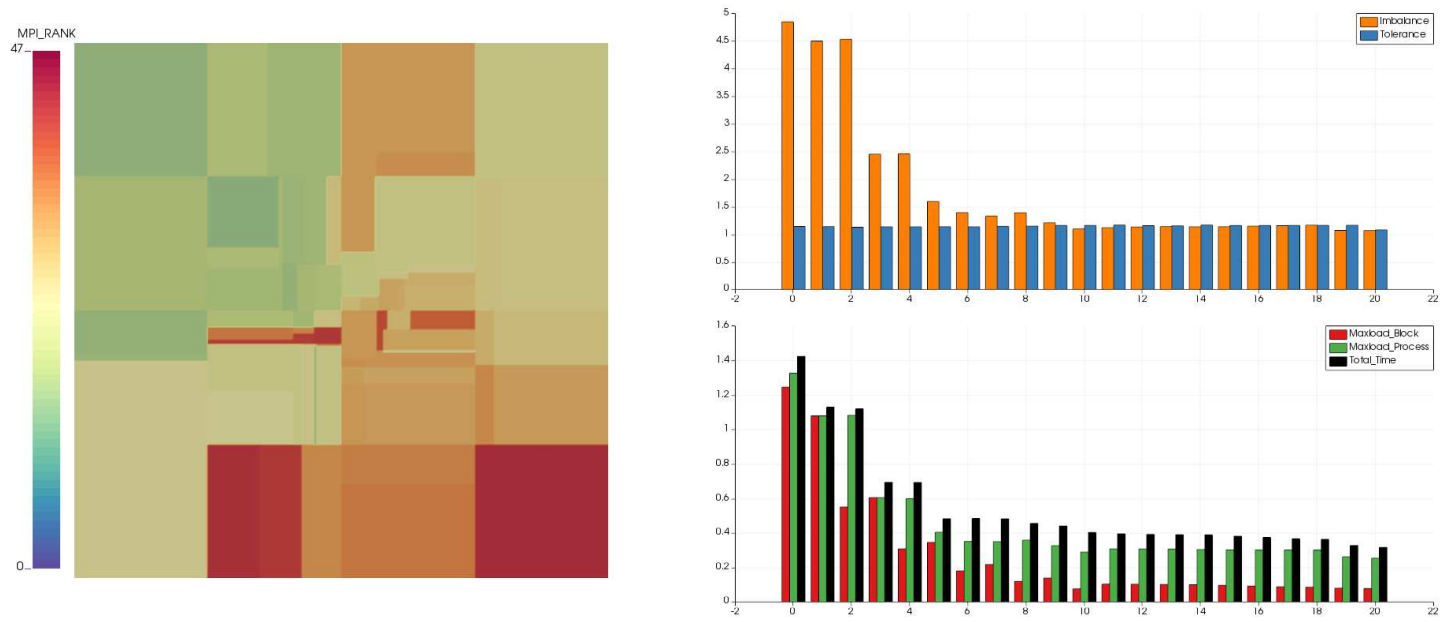

Figure 6.7: Simulation using 48 processes with a high computational load in the center of the domain, see section 6.2.2. Using the threshold parameter $\gamma$ as defined in remark 5.2.7 the blocks are adaptively resized and assigned to processes. It takes several steps at the beginning of the simulation to find the optimal sizes. As long as the load-imbalance is lower than the tolerance neither bisection nor reassignment of blocks using graph-partitioning are performed. In the top plot the actual load-imbalances is colored orange and the tolerance blue. In the bottom plot black denotes the total wall time, green the maximum load among the processes and red the maximum load among the blocks.

\subsubsection{Gaussian Distribution of Particles}

In the domain $\Omega$ a number $N_{p}=10 \times N_{\text {MPI }} \times N_{\text {OpenMP }}$ of spherical particles are embedded at random coordinates. The particle positions follow a normal distribution with a mean of $\mu_{d}=\frac{N_{d}}{2}$ and a standard deviation $\sigma_{d}=\frac{N_{d}}{8}$ for the dimensions $d \in\{x, y, z\}$ and the particle sizes again follow a normal distribution with a mean radius of $\mu_{r}=10$ and a standard deviation $\sigma_{r}=2$. That means the particles are clustered near the center of the domain $\Omega$ and the phase fields form many multiple junctions, which leads to severe load-imbalances when using a simple domain decomposition (see figure 6.5b. Figure 6.7 shows the load-imbalance over the first 2000 time steps. In the beginning the load-imbalance is high and the optimal tolerance as defined in remark 5.2.7 is much lower. Blocks in the center of the domain, where the computational load is high, are split, while blocks on the outside are merged 
together. It takes multiple steps to decrease the load of the most expensive block that prevents the graph-partitioner from obtaining a well-balanced partitioning. After 1000 time steps the graph-partitioner is able to find a partitioning that satisfies the tolerance and no bisection and graph-partitioning is performed until the loadimbalance exceeds the tolerance after 1800 steps, when the block sizes are adapted again and a new partitioning is calculated.

The benchmark results in figure 6.8 show that especially for a large number of cores the performance deteriorates without load-balancing. The utilization of loadbalancing consisting of the bisection of blocks and either METIS or the greedy graph-partitioner to assign blocks to process provides a high weak-scaling efficiency. In figure 6.9 the performance using dynamic resizing of blocks is compared to static block sizes using METIS as the graph-partitioner. Bisection of blocks results is the best performance and further refinement of blocks will not deliver better results for the static block sizes as figure 6.10 shows that the overhead created by small blocks already raised the average load $\tilde{W}$ per partition over load of the maximum partition using dynamic resizing of blocks. Figure 6.11 shows the performance using different numbers of OpenMP threads, while still using the same number of total cores. For a small problem size with a total of 24 cores the hybrid parallelization with 6 threads per MPI process shows the best performance. In this case the 4 processes are assigned to 8 blocks with similar computational load because the particles are clustered near the center of the domain. Therefore a good load-balance is achieved without using the load-balancing algorithm in section 5.2.6, which means that less overhead is created by the bisection of blocks. The situation is the same for up 48 cores, when using 12 threads per process. For larger system sizes the results show a similar performance for 4 and 6 threads per process. A pure MPI-parallelization shows a slightly worse performance, except for 384 cores. For large system sizes the performance deteriorates significantly when using 24 threads per MPI-process. The current implementation only allows one thread to use MPI calls, which decreases the rate of parallelism. Hybrid-parallelism is further investigated for the simulation of $\mathrm{Mg}-\mathrm{Al}$ alloy solidification in section 6.3. 


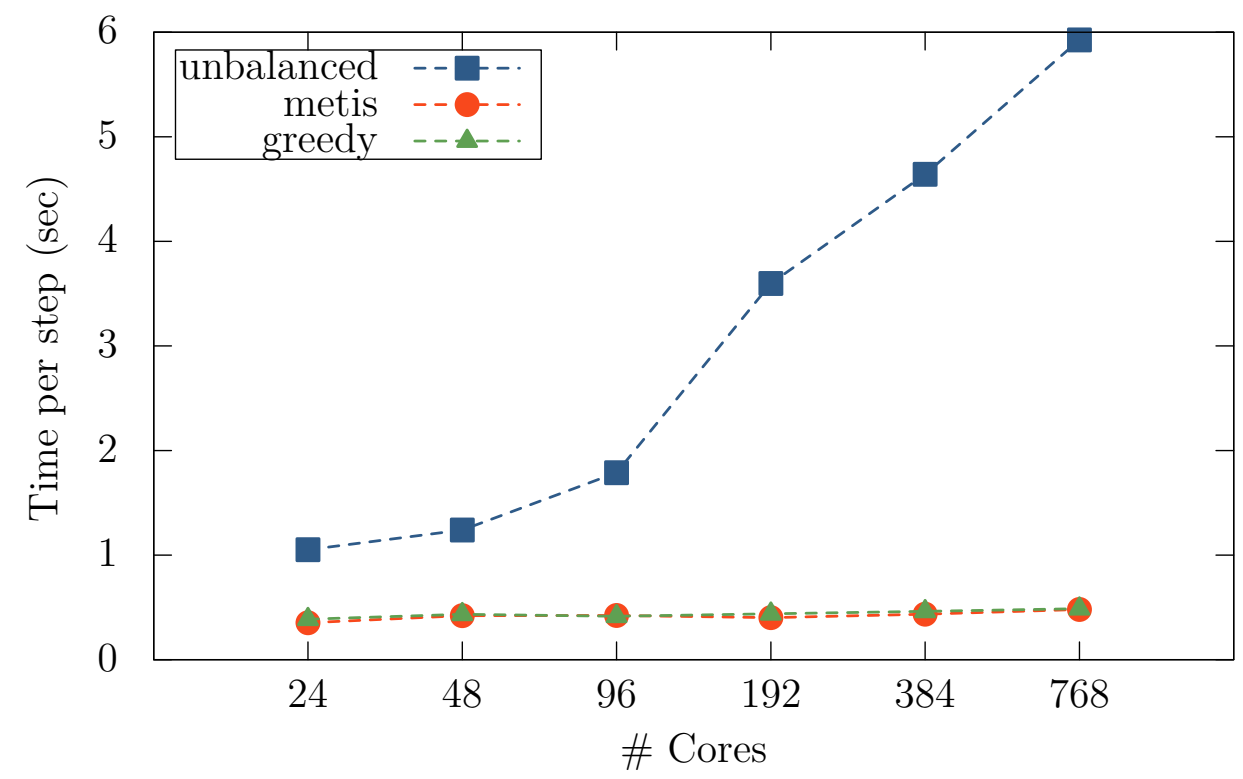

Figure 6.8: Grain growth scaling benchmark for a Gaussian particle distribution, see section 6.2.2. In this case the load-imbalances are very significant and with load-balancing the parallelization does not scale. With load-balancing using either METIS or the greedy graph-partitioner provided with OpenPhase we observe a proper weak-scaling performance.

\subsubsection{Moving Particles}

In the tests above grains are stationary and therefore the distribution of the computational load does not change significantly over time. In this test we use a similar setup as in section 6.2 .2 , but the grains move one grid point along the $x$-direction every 5 time steps, in order to create a dynamic load distribution. The computational domain is a perfect cube consisting of $N_{b}$ blocks in each direction, that have an edge length of $N_{e} . N_{b}$ and $N_{e}$ are determined by

$$
\begin{aligned}
& N_{b}=\max \left(\left\lceil N_{\mathrm{MPI}}^{\frac{1}{3}}\right\rceil, 3\right), \\
& N_{e}=\left\lfloor\frac{\left\lfloor\left(2 \cdot 64^{3} N_{\mathrm{MPI}} N_{\text {OpenMP }}\right)^{\frac{1}{3}}\right\rfloor}{N_{b}}\right\rfloor
\end{aligned}
$$

with the number of MPI processes $N_{\text {MPI }}$ and the number of OpenMP threads per process $N_{\text {OpenMP. }}$ In the simulation with load-balancing an initial ten load-balancing 


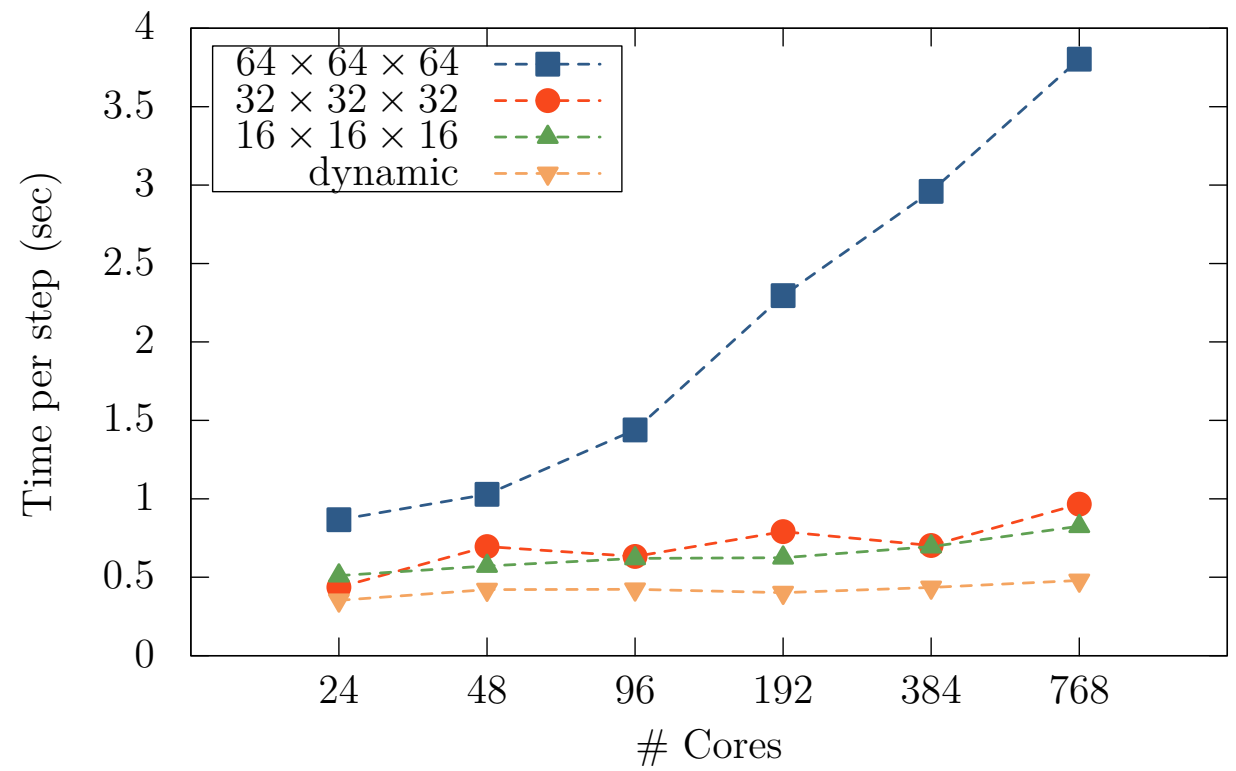

Figure 6.9: Grain growth scaling benchmark for a Gaussian particle distribution, see section 6.2.2. Comparing the dynamic splitting and merging of blocks to static block sizes using METIS for graph-partitioning in all cases. Using dynamic block sizes results in the best performance in this test.

steps are used before the start of the benchmark in order to let the load-balancing algorithm adapt to the initial configuration. In figure 6.12 we see the wall times per time step on 192 processes with dynamic load-balancing as well as for a simple static domain decomposition. We notice that the load-balancing steps can take as long as about ten time steps in this example. The simulation uses $\lambda=0.5$ for equation 5.32 and balances every $m=100$ time steps if inequality 5.34 is not fulfilled or if a block $B$ exists with

$$
W_{B} \geq 2(1+\gamma) \tilde{W}
$$

which triggers the secondary load-balancing steps in steps 89 and 192. Figure 6.13 shows the scaling behavior for the average wall time per step $\tilde{T}$, which includes the time used for load-balancing. 


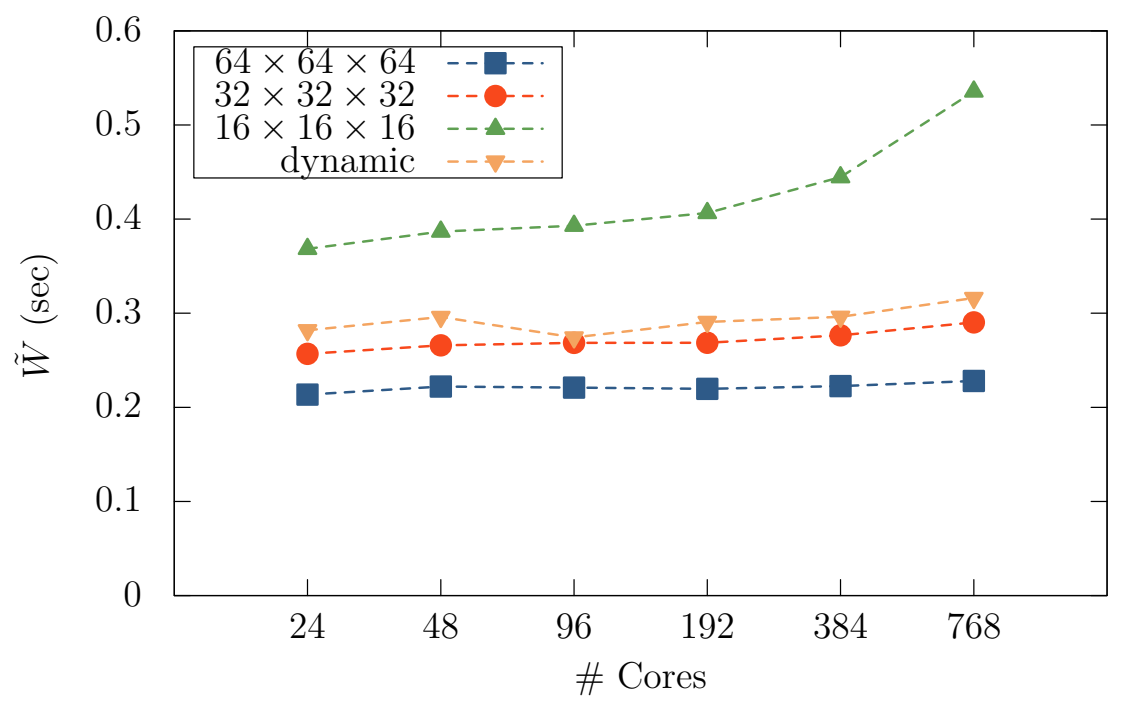

Figure 6.10: Grain growth scaling benchmark for a Gaussian particle distribution, see section 6.2.2. This shows the average load $\tilde{W}$ per time step using different block sizes. For $16 \times 16 \times 16$ blocks the average load $\tilde{W}$ already exceeds the time per step of the dynamic block sizes in figure 6.9 , which means that using even smaller blocks will not perform better than using the dynamic block sizes.

\subsection{Mg-Al Alloy Solidification}

An important property of $\mathrm{Mg}-\mathrm{Al}$ alloys is their low density. Therefor they are commonly used for lightweight structural applications in automotive and aerospace engineering and in high-end consumer electronics. However, due to their low nobility $\mathrm{Mg}-\mathrm{Al}$ alloys are sensitive to corrosion, especially in contact with other metals. $\mathrm{Mg}$ $\mathrm{Al}$ cast alloys usually consist of a primary $\mathrm{HCP}-\mathrm{Mg}-\alpha$ phase, which solidifies in a dendritic shape. These dendrites are then enclosed during the solidification by an interdendritic eutectic, which consists mostly the inter-metallic $\mathrm{Mg}_{17} \mathrm{Al}_{12}$ - $\beta$-phase. One of the many strategies to inhibit the galvanic corrosion in $\mathrm{Mg}-\mathrm{Al}$ alloys is to prevent networks of primary $\alpha$-phase grains from forming. Ensuring a closed shell of $\beta$-phase around the individual primary dendrites, significantly increased corrosion resistance [65]. In order to understand the microstructure evolution of as-cast $\mathrm{Mg}$ $\mathrm{Al}$ alloys during solidification and to be able to predict the connectivity of the 


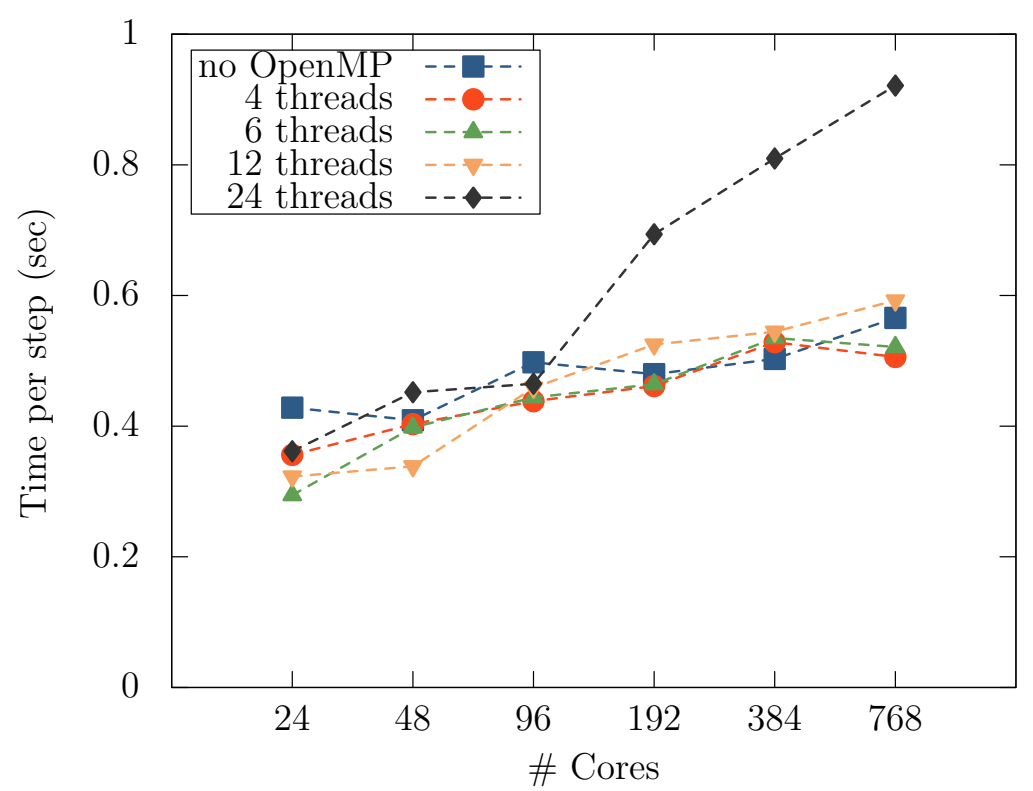

Figure 6.11: Grain growth scaling benchmark for a Gaussian particle distribution, see section 6.2.2. Comparison of the performance using a varying number of OpenMP threads per MPI-process.

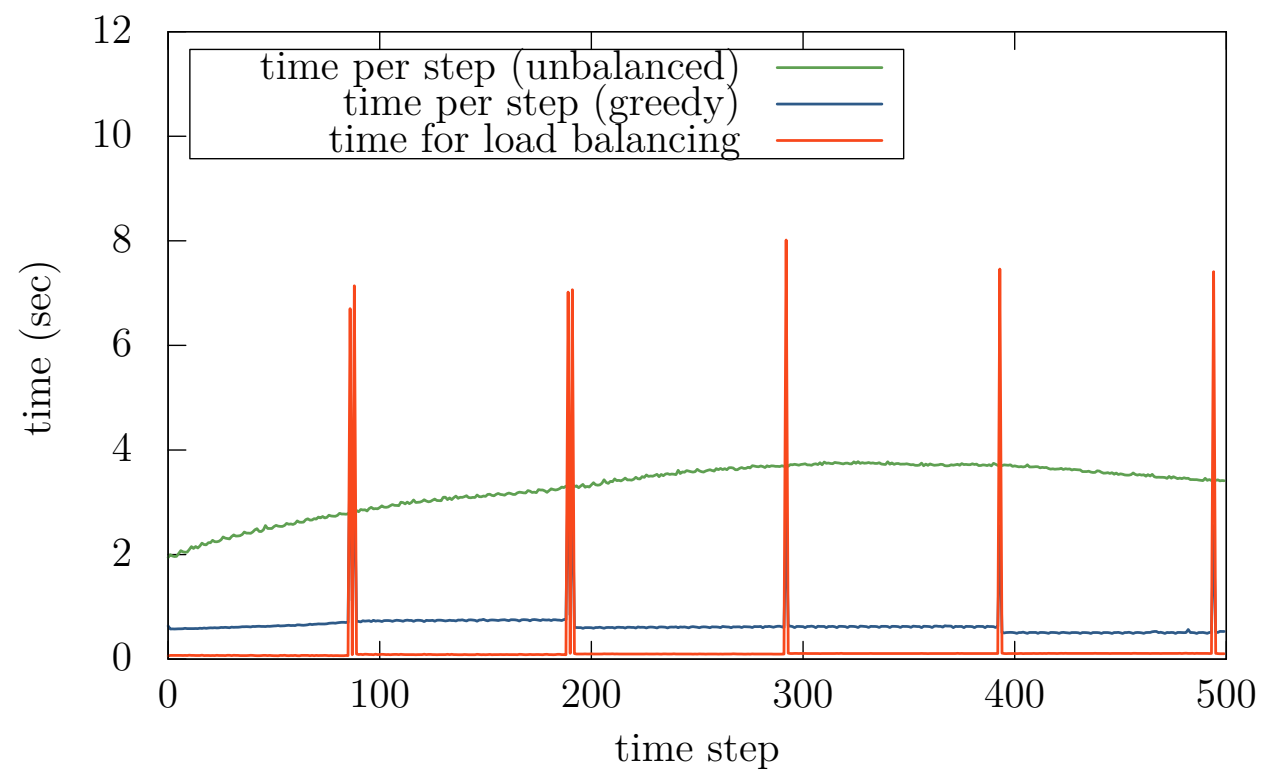

Figure 6.12: Comparison of the wall time per time step for the simulation of moving particles in section 6.2.3 with load-balancing and without load-balancing for 192 processes. 


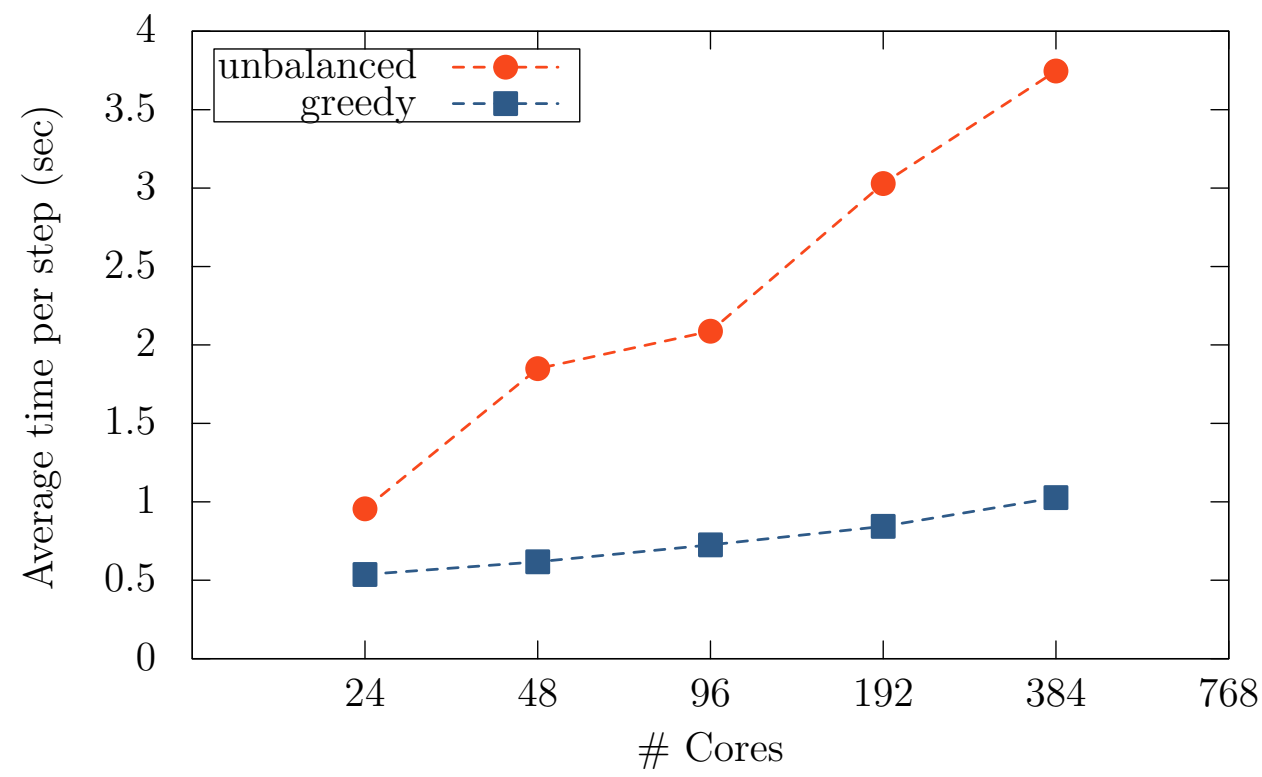

Figure 6.13: Scaling behavior for example 6.2.3 using the greedy graph partitioner and a simple domain decomposition using the average wall time per step $\tilde{T}$, which includes the time used for load-balancing.

$\beta$-phase depending on process parameters as composition and cooling rate, phase field studies have been carried out and will be briefly explained below. Figure 6.14 shows a simulation of $\mathrm{Mg}-\mathrm{Al}$ alloy solidification on a grid of $300^{3}$ grid points. Further information can be found in previous publications $[66,67,1]$.

The parallelization techniques that were explained in chapter 5 are used to enable a large scale simulation of a complete solidification process of a $\mathrm{Mg}-5 \mathrm{at} . \% \mathrm{Al}$ alloy. This simulation shows the sequential nucleation and growth of primary $\alpha$ - and in a later stage secondary $\beta$-phase. To recover the physics behind the process, multiple solvers have to be used. The phase field evolution with the driving-force evaluation has to be calculated. This is of course dependent on the local concentration of $\mathrm{Al}$ and the temperature. While the diffusion equation is solved to model the evolution of the composition field, the heat is assumed to diffuse orders of magnitude faster than the diffusion and is modeled using a heat-balance equation. The inclusion of the latent heat term creates the necessity to know at every time step how much phase was transforming in the whole domain. The nucleation events were modeled such that a list of virtual particles was generated pseudo-randomly with a position 


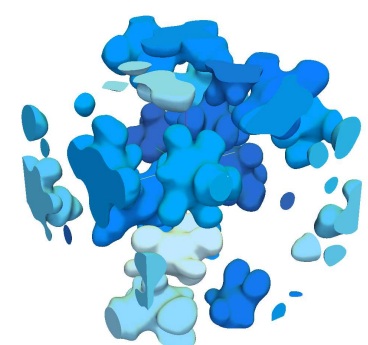

(a)

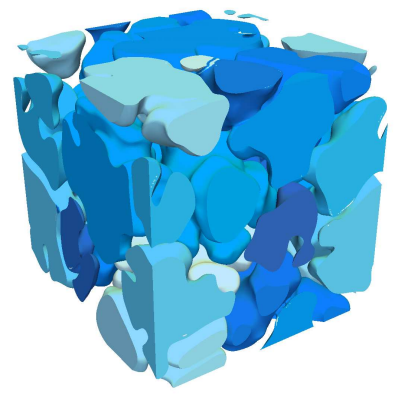

(d)

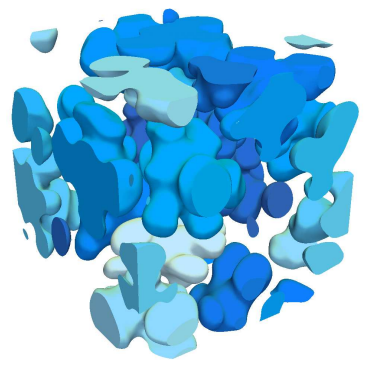

(b)

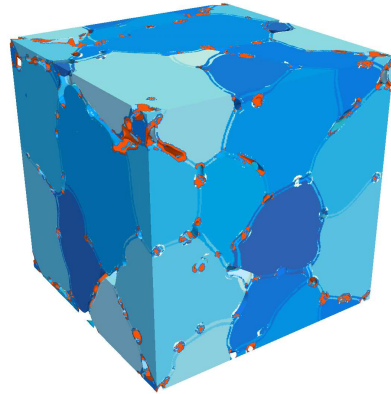

(e)

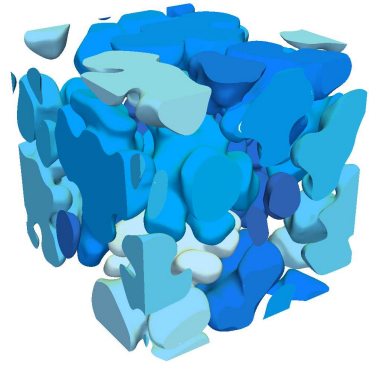

(c)

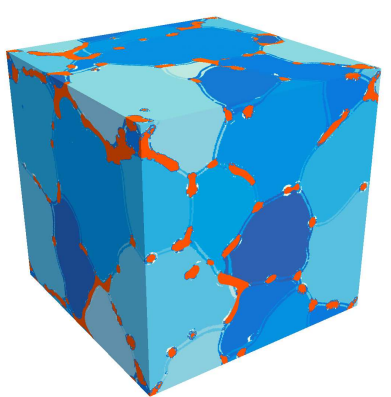

(f)

Figure 6.14: Simulation of $\mathrm{Mg}-\mathrm{Al}$ alloy solidification. At first the $\alpha$-phase grows, then the secondary $\beta$-phase nucleates and solidifies in the Al-rich gaps between the $\alpha$-phase grains.

and size each. Then at every time step it is checked if a nucleation is taking place on that particle by evaluation of the local composition and temperature. In case of a high enough under-cooling, a new grain is planted at the exact position of that particle. The implementation of the chemical driving force will be explained in the following section. 


\subsection{Chemical Driving-forces and Diffusion}

As mentioned in section 2.2 additional energy contributions can be considered. As in [6] a chemical energy density can be given by

$$
f^{\mathrm{CH}}=\sum_{\alpha=1}^{N} \phi_{\alpha} f_{\alpha}\left(c_{\alpha}\right)+\lambda\left[c-\sum_{\alpha=1}^{N}\left(\phi_{\alpha} c_{\alpha}\right)\right],
$$

with bulk free energies $f_{\alpha}\left(c_{\alpha}\right)$ of the individual phases.

The vector $c$ is the vector of the overall concentrations in a mixture and $c_{\alpha}$ is the vector of its concentrations in the phase $\alpha$. $\lambda$ is a generalized chemical potential, that introduced as a Lagrange multiplier in order to conserve the mass balance between the phases

$$
c=\sum_{\alpha=1}^{N} \phi_{\alpha} c_{\alpha}
$$

The evolution equation for the concentration given by

$$
\dot{c}=\nabla \cdot\left\{\sum_{\alpha} \phi_{\alpha}\left[D^{\alpha} \nabla c_{\alpha}\right]+\sum_{\alpha, \beta} J_{\alpha \beta}\right\}
$$

with the diffusion matrices $D^{\alpha}$ in the individual phases $\alpha$ for the pairwise interaction of chemical components $c_{i}$ and $c_{j}$. The last term in the right-hand side is the antitrapping current $J_{\alpha \beta}$ which eliminates the numerical solute trapping due to the diffusiveness of the interface, also see [6].

As above we get

$$
\dot{\phi}_{\alpha}=\sum_{\substack{\beta=1 \\ \beta \neq \alpha}}^{N} \frac{\mu_{\alpha \beta}}{N}\left[\sum_{\substack{\gamma=1 \\ \gamma \neq \beta}}^{N}\left(\sigma_{\beta \gamma}-\sigma_{\alpha \gamma}\right) I_{\gamma}+\frac{\pi^{2}}{8 \eta} \Delta g_{\alpha \beta}\right],
$$

with

$$
\Delta g_{\alpha \beta}=-f_{\alpha}\left(c_{\alpha}\right)+f_{\beta}\left(c_{\beta}\right)+\lambda\left(c_{\alpha}-c_{\beta}\right),
$$

and $\lambda=\frac{\partial f_{\alpha}}{\partial c_{\alpha}}$ according to $[68]$. 


\subsection{Scaling Benchmark for Mg-Al Alloy Solidification}

Compared to the grain growth simulation in section 6.1 this simulation requires more work. It includes the calculation and averaging of the driving force based on the local composition of magnesium and aluminum and the temperature, as described in section 2.4. In addition to that the diffusion of aluminum in magnesium needs to be calculated as well. The calculation of the Laplacian of the phase fields and the concentrations require stencils that access the next neighbors. In this case we need $\frac{2}{3} \eta$ boundary layers for each of the driving force averaging steps 2.27 and 2.28 , one for the tagging of grid points as interface or near-interface points as well as one for the diffusion equation 6.8 and one additional layer is needed to compute the anti-trapping current $J_{\alpha \beta}$, the stencil operation in equation 2.25 can be computed simultaneously and does not increase the halo size. With an interface width $\eta$ of 5 grid points a total of 6 grid points is needed for the driving force averaging, which makes a halo size of 9 grid points necessary.

For the scaling tests we use a domain $\Omega$ consisting of $N$ blocks with

$$
N=2 N_{\mathrm{MPI}},
$$

where $N_{\text {MPI }}$ is the number of MPI processes. Each block has a size of $48 \times 48 \times 48$ grid points plus nine boundary points in each direction in single threaded benchmarks, with two threads per process the size of each blocks is $96 \times 48 \times 48$ and with four threads it is $96 \times 96 \times 48$. The blocks are again assembled in a way so that the computational domain $\Omega$ forms a cuboid with the minimal surface area. Therefore we have 5308416 grid points for 24 cores and go up to 169869312 grid points for 768 cores.

\subsubsection{Uniform Distribution of Particles}

As the computational complexity in test is significantly higher compared to the normal grain growth example in section 6.1 we only embed $N_{p}=2 \times N_{\text {MPI }} \times N_{\text {OpenMP }}$ spherical particles in the domain $\Omega$. The particles are distributed uniformly in the 


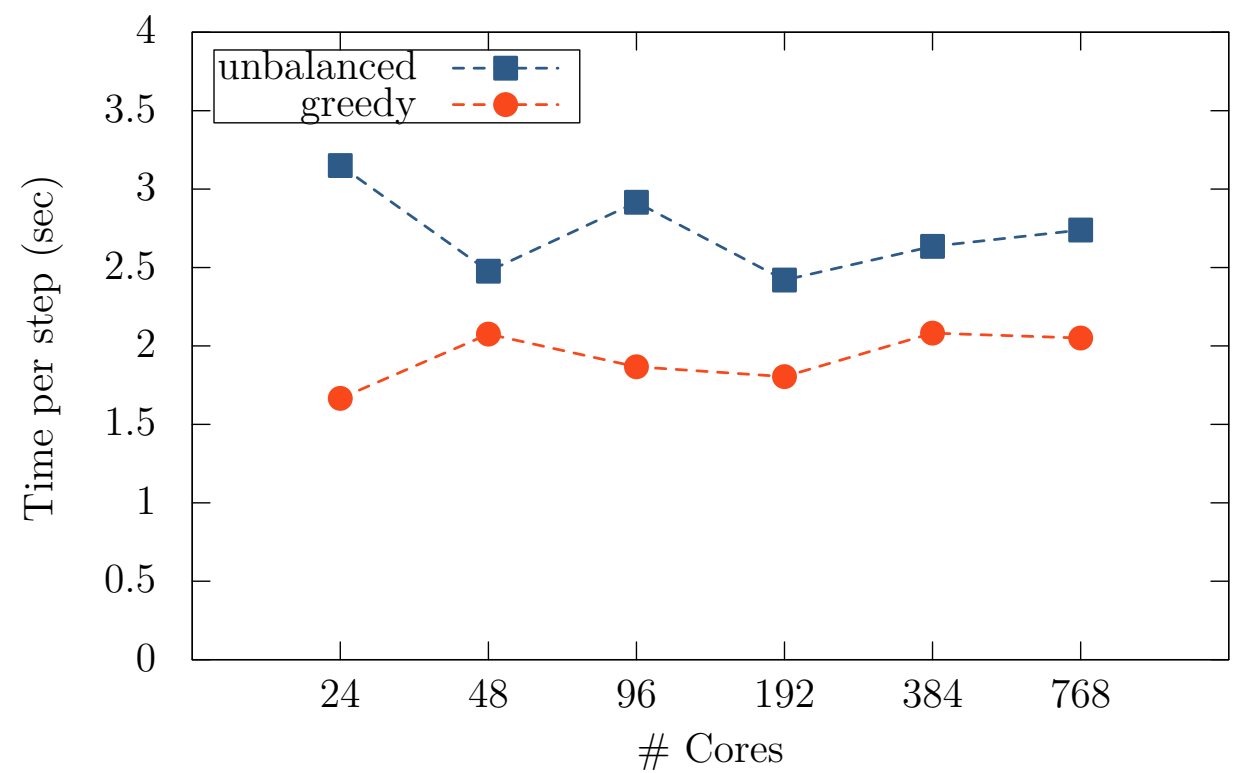

Figure 6.15: $\mathrm{Mg}-\mathrm{Al}$ alloy solidification benchmark for a uniform particle distribution, see section 6.5.1. Although the particles are distributed uniformly in the domain some small load-imbalances occur and load-balancing improves the performance.

domain and the particle sizes follow a normal distribution with a mean radius of $\mu_{r}=10$ and a standard deviation $\sigma_{r}=2$. This test represents a simulation without significant load imbalances.

At first we take a look at the performance for an uniform distribution of particles as mentioned in section 6.5.1. The results are depicted in figure 6.15. As for the normal grain growth we again observe that load-balancing improves the performance even for a uniform particle distribution without significant load-imbalances.

\subsubsection{Gaussian Distribution of Particles}

In the domain $\Omega$ a number $N_{p}=2 \times N_{\text {MPI }} \times N_{\text {OpenMP }}$ of spherical particles are embedded at random coordinates. The particle positions follow a normal distribu- 
tion with a mean of $\mu_{d}=\frac{N_{d}}{2}$ and a standard deviation $\sigma_{d}=\frac{N_{d}}{8}$ for the dimensions $d \in\{x, y, z\}$ and the particle sizes again follow a normal distribution with a mean radius of $\mu_{r}=10$ and a standard deviation $\sigma_{r}=2$. That means the particles are clustered near the center of the domain $\Omega$ and the phase fields form many multiple junctions, which leads to severe load-imbalances when using a simple domain decomposition (see figure 6.5b).

For the Gaussian particle distributions the scaling results are presented in figure 6.16. As in the grain growth benchmark in section 6.2.2 load-balancing drastically improves the performance. Without load-balancing a very poor weak scaling behavior is observed. With load-balancing, however, the time per step remains close to 5 seconds. Using the hybrid approach is beneficial in this case as seen in figure 6.17. Using 6 or 12 threads yields the best results across all problem sizes. In contrast to the grain growth benchmark in section 6.2.2 the workload per time step in the example is high compared to the amount of communication. This makes a hybridparallelization viable even when only on thread is allowed to make MPI calls. Also we can see that without OpenMP the time per step shoots up at 768 cores. This is caused by a single block that cannot be further split but remains so costly as to cause a severe load-imbalance. Also the hybrid parallelization results in larger block sizes as mentioned in remark 5.2.8, which causes less duplicated computations on the wide halo and thus is more efficient. For 24 threads per MPI process the results for 96 or less cores, i.e. 1 to 4 MPI-processes or 2-8 blocks, are on par with 6 or 12 threads per process. In these cases every blocks has about the same computational load and thus a good load-balance is achieved without resizing or reassignment of blocks | the same is true for up 48 cores when using 12 threads per MPI-process or for 24 cores when using 6 threads per MPI-process. Using more cores, however, load-imbalances between blocks occur and blocks are split and reassigned. Some blocks become to small for an efficient OpenMP parallelization using 24 threads, which causes the deterioration of the performance seen in figure 6.17. 


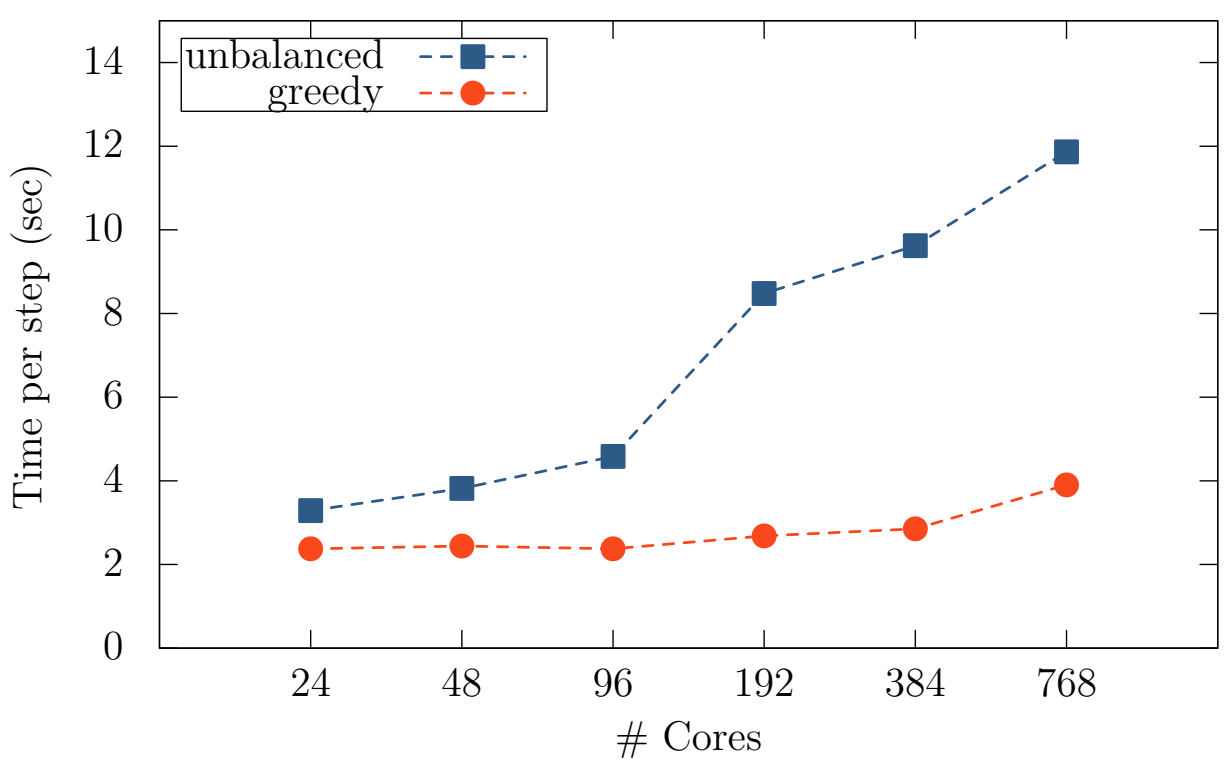

Figure 6.16: $\mathrm{Mg}-\mathrm{Al}$ alloy solidification benchmark for a Gaussian particle distribution, see section 6.2.2. In this case the load-imbalances are very significant and the performance deteriorates without load-balancing.

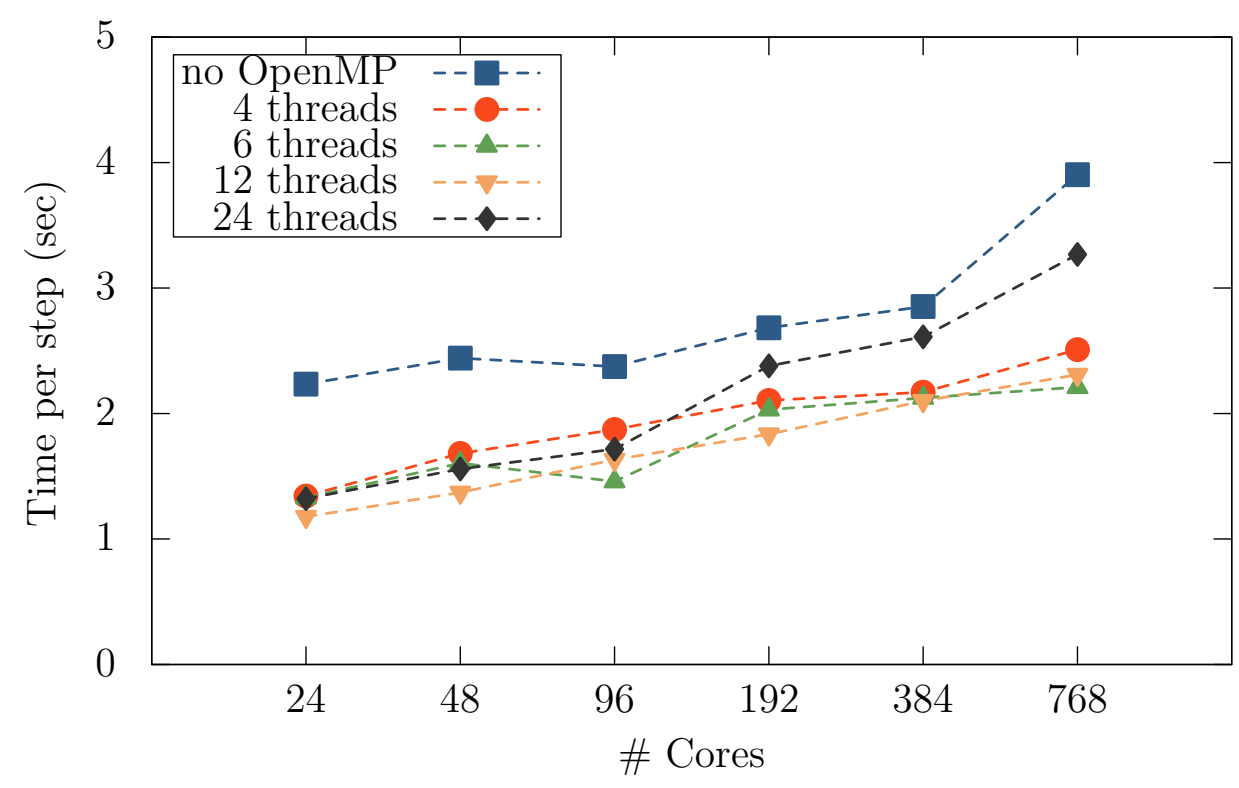

Figure 6.17: $\mathrm{Mg}-\mathrm{Al}$ alloy solidification benchmark for a Gaussian particle distribution, see section 6.2.2. Comparison of the performance with load-balancing using a varying number of OpenMP threads per MPI-process. 6 or 12 threads per MPI process provide the best performance in this test. 


\subsubsection{Uniform Particle Distribution in one Quadrant of the Domain}

The following example was already presented in [1]. In this set-up we use a grid with a number of blocks equal to the number of MPI processes, the size of each block is $25[$ OpenMP Threads] $\times 50 \times 50$. This ensures that the computational domain is the same for the same number of processors

$$
p=[\text { MPI Processes }][\text { OpenMP Threads }] .
$$

In one quadrant of this domain we place a number of

$$
n_{\text {particles }}=\frac{[\text { MPI Processes }][\text { OpenMP Threads }]}{8}
$$

$\alpha$-phase particles at randomized positions to intentionally create an imbalance in the computational load. We use particles with a diameter of 20-40 grid points, regions with multiple phase fields are formed, their presence is the major contribution to the computational load. Thus, this is not a real weak scaling test as the workload does not scale linearly with the number of processors.

For load-balancing we let the block distribution adapt for 20 time steps and then compute 10 time steps. The results are shown in figure 6.18.

We note that load-balancing can significantly improve the performance. With loadbalancing we also observe that the performance for large increases when more OpenMP threads and less MPI processes are used. In the hybrid approach we have a smaller number of larger blocks, which reduces the overhead created by the wide halo. Also as the initial blocks are larger, blocks can be split additional times, which allows a reduction of the relative load-imbalance

$$
\gamma=\frac{\omega_{o p t}}{\tilde{W}} .
$$

Without load-balancing we cannot see this behavior as the wall time is based primarily on load-imbalances between processes. 


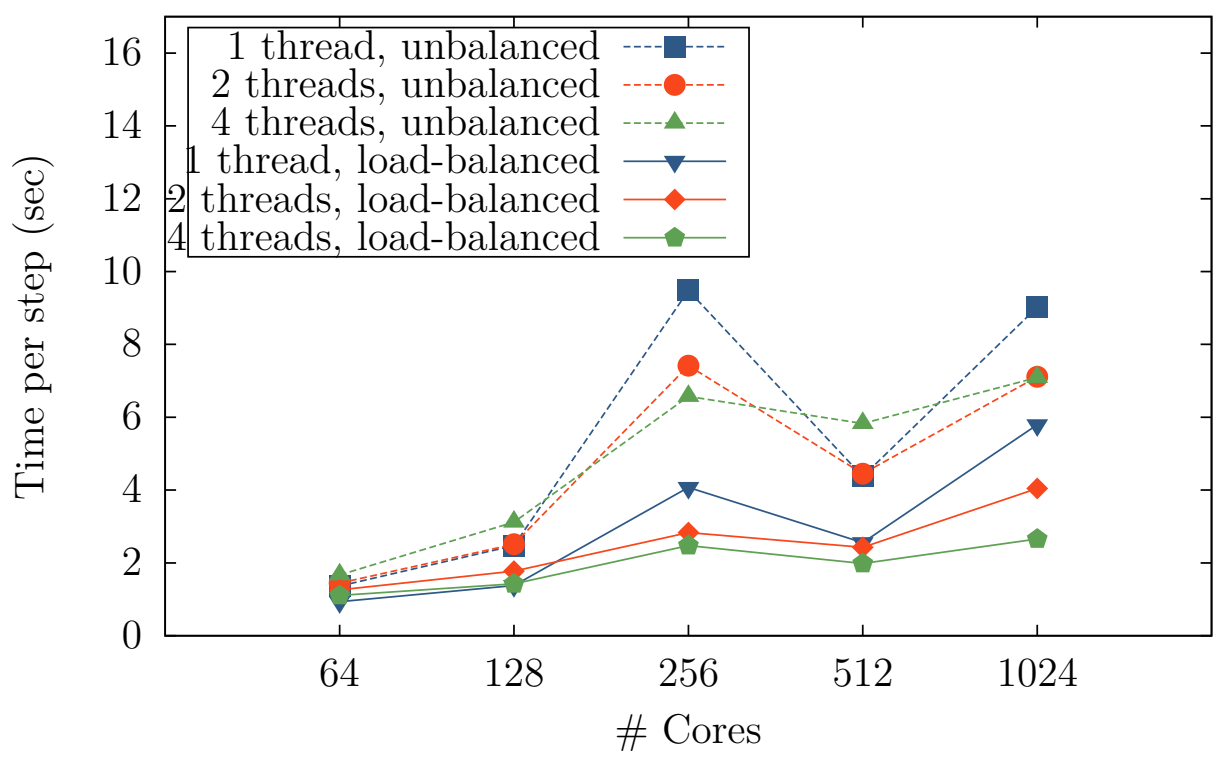

Figure 6.18: Comparison of the wall time in seconds per time step for the benchmarks in 6.5.3 with or without load-balancing and a varying number of OpenMP Threads. The number of MPI processes is chosen so that the total number of used processors is independent on the number of OpenMP Threads.

\subsection{Effect of Solidification on the Fluid Flow}

In [2] a study on the evolution of the permeability in a Mg-5\%Al-cast alloy was presented. This simulation showcases the coupling between the multiphase field method and the Lattice-Boltzmann method. In a first step the evolution of the dendritic microstructure was simulated under purely diffusive conditions, see also 6.3 and $[66,67]$. Then the Lattice-Boltzmann method was utilized to simulate a fluid flow through a channel that is obstructed by the dendrites.

\subsubsection{Simulation Setup}

In the first step the solidification of $\mathrm{Mg}-5 \% \mathrm{Al}$ cast alloy was simulated in a domain $\Omega$ with $1000 \times 1000 \times 250$ grid points. The grid spacing $\Delta x$ is $10^{-6} \mathrm{~m}$ and the initial 
temperature is $888 \mathrm{~K}$ and the cooling rate is $10 \mathrm{~K} \mathrm{~s}^{-1}$. A total of 42 grains have been planted semi-randomly on one side of the larger surfaces of the domain with a random orientation of the crystal structure, i.e. the seed points are randomly inserted with a minimum distance of 80 grid points between each other. The simulation has been conducted on 400 cores, which are 25 nodes with 16 cores each. Each node used exactly one process with exactly one block and no load-balancing was utilized in this simulation. Parallelization within a node was only using the shared-memory parallelization using OpenMP, see section 4.1.2. Of the 128GB of available memory on each node about 70GB were necessary for the computation.

As the dendrites were only planted on one side of the domain in order to safe computation time, the simulation domain is mirrored to form a channel. Multiple stages during the solidification under purely diffusive conditions are depicted in figure 6.19. All grains grow in a dendritic manner and obstruct more space as the solidification continues.

\subsubsection{Darcy's Law}

At various points of the solidification simulation checkpoints were created that serve as structural input for Lattice-Boltzmann simulations, which aim to compute the permeability of the channel. The permeability $\kappa\left(\mathrm{m}^{2}\right)$ of a channel can be described by Darcy's Law [69] as

$$
Q=-\frac{\kappa A \Delta p}{\mu L_{x}}
$$

with the total discharge $Q\left(\mathrm{~m}^{2} / \mathrm{s}\right)$, the cross-sectional area $A\left(\mathrm{~m}^{2}\right)$, the pressure drop $\Delta p\left(\mathrm{~kg} / \mathrm{ms}^{2}\right)$ and the dynamic viscosity $\mu(\mathrm{kg} / \mathrm{ms})$ as well as the length of the channel $L_{x}(\mathrm{~m})$. Using the total volumetric discharge $q=Q / A$ and we obtain a simple expression of the permeability coefficient

$$
\kappa=-\frac{\mu q L_{x}}{\Delta p} .
$$




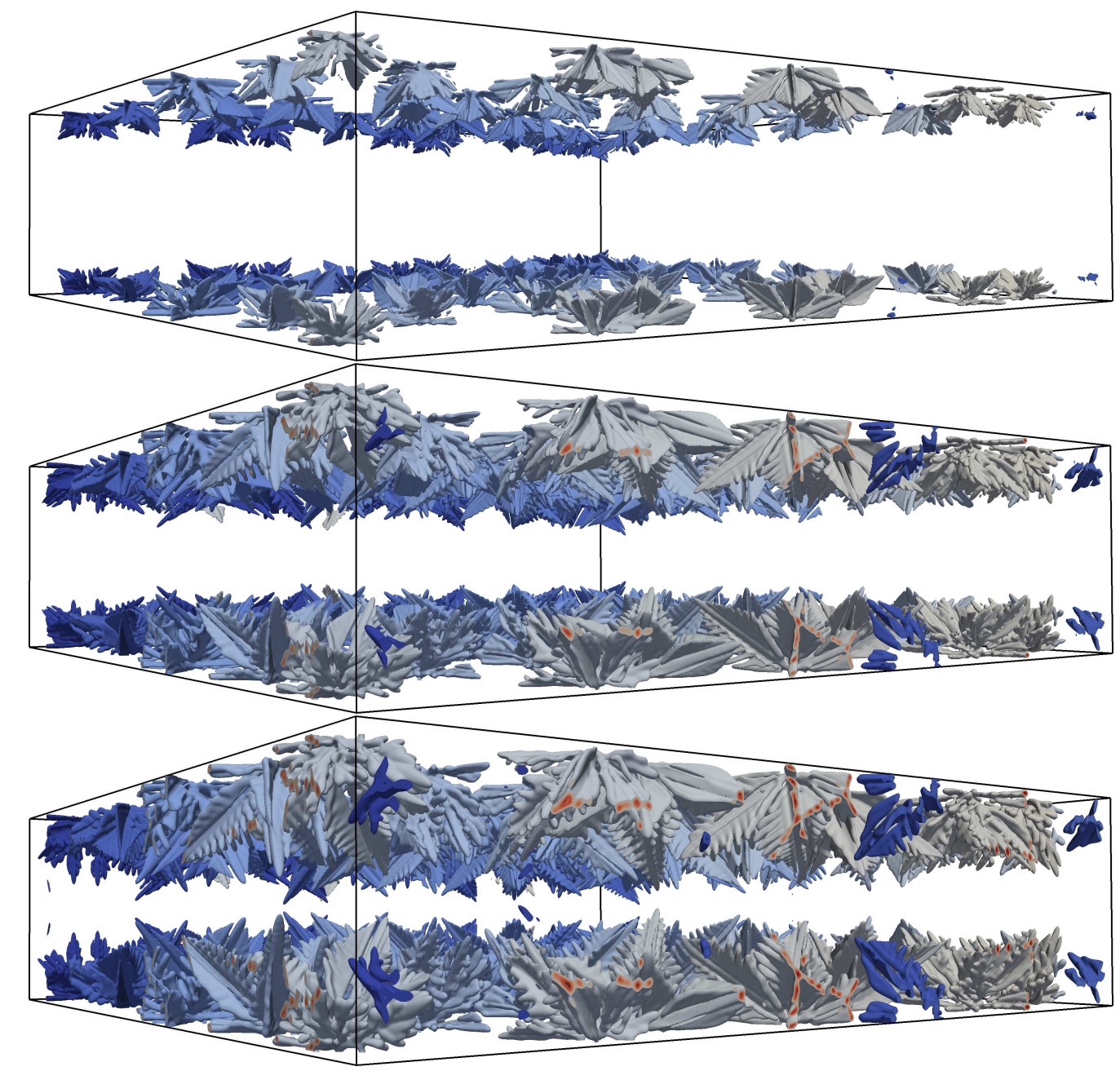

Figure 6.19: Different time stages of the dendritic solidification of an $\mathrm{Mg}-\mathrm{Al}$ alloy as described in section 6.6.1. 


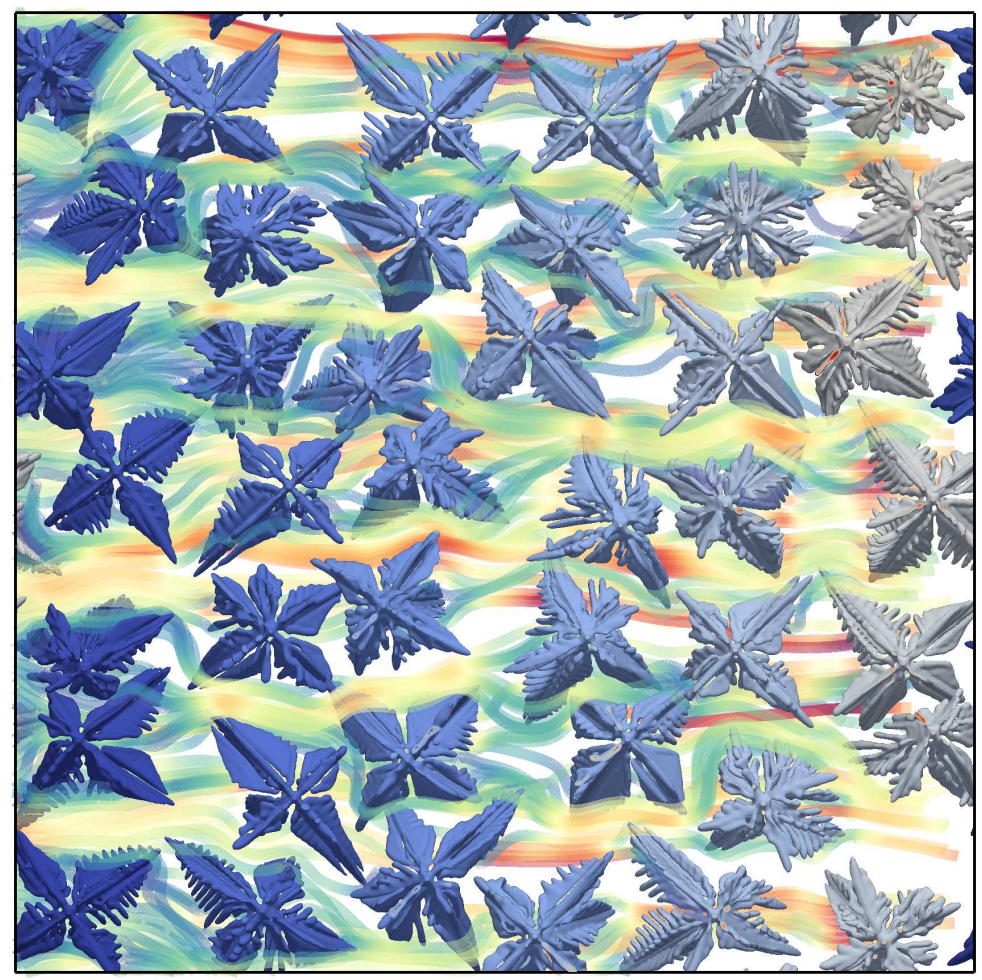

Figure 6.20: View from the top of the domain depicting the stream lines of the flow through the channel along the dendrites.

An external body force $F=\rho g \delta(x)$ is imposed in the Lattice Boltzmann simulation to describe the pressure drop using the density $\rho$, the magnitude of the body force $g$ and the Dirac delta function $\delta(x)$, that ensures that the body force is applied at the entrance of the channel. With $\rho g \delta(x)=-\nabla p$ we obtain

$$
\Delta p=\int \nabla p d x=-\int \rho g \delta(x) d x=-\rho g
$$

Therefore Darcy's Law can be expressed as

$$
\kappa=\frac{q \mu L_{x}}{\rho g}
$$




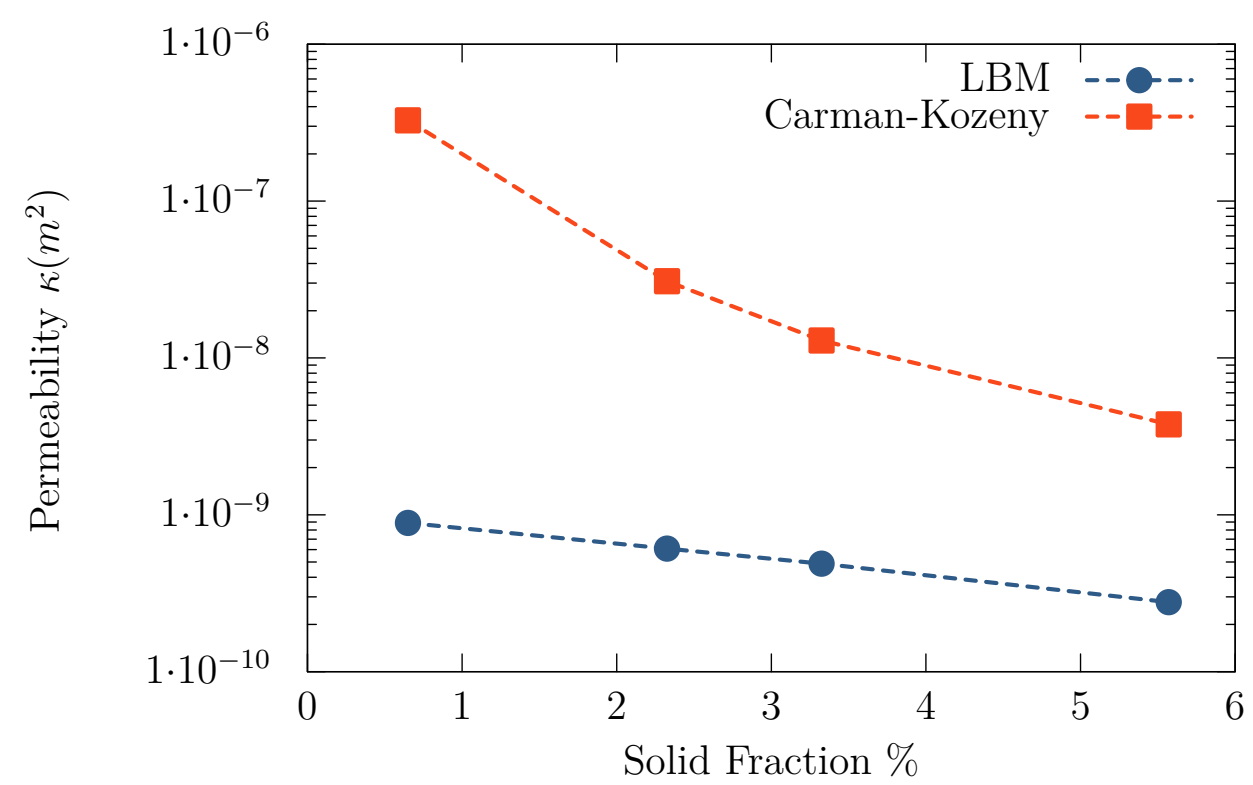

Figure 6.21: Comparison of the permeability coefficient obtained by the LatticeBoltzmann simulation and the estimate by the Carman-Kozeny equation.

In order to ensure that the fluid velocity is small compared to the sound speed, which in LB units is $c_{s}=\frac{1}{\sqrt{3}}$, the parameter $g$ is set to

$$
g=\frac{8 \mu L_{x} u_{\max }}{\rho L_{z}^{2}}
$$

according to [70] with the threshold velocity $u_{\max }=10^{-4}$ in LB units. The force is applied using the exact difference method as described in 3.2. In the simulation the following parameters are used:

\begin{tabular}{l|r|r} 
Parameter & LBM units & SI units \\
\hline$\rho$ & 1 & $1587 \mathrm{~kg} / \mathrm{m}^{3}$ \\
$\mu$ & 0.1 & $1.587 \cdot 10^{-10} \mathrm{~kg} / \mathrm{ms}$ \\
$\mathrm{g}$ & $1.28 \cdot 10^{-6}$ & $1.28 \cdot 10^{-18} \mathrm{~m}^{2} / \mathrm{s}^{2}$
\end{tabular}

The density of liquid magnesium close to the melting point is used for the fluid density [71]. The calculated melt flow is depicted in figure 6.20 by stream lines of the flow through the dendritic microstructure. 


\subsubsection{Carman-Kozeny Equation}

The Carman-Kozeny equation gives an estimate of the permeability coefficient for laminar flows

$$
\kappa=\frac{\left(1-f_{s}\right)^{2}}{\epsilon^{2} f_{s}^{3}}
$$

$\kappa$ for a dendritic microstructure using the ratio between surface and volume $\epsilon=\frac{A}{V}$ and the solid-fraction $f_{s}$ in the channel $[72,73]$. Figure 6.21 depicts the comparison of the estimate given by the Carman-Kozeny equation and the permeability coefficient $\kappa$ obtained by Darcy's Law using Lattice-Boltzmann simulations at different stages of solidification. Darcy's Law gives a significantly lower permeability compared to the Carman-Kozeny. For a solid fraction of 5\% the Carman-Kozeny equation gives a an estimate that is approximately twenty times higher than the value obtained from the Lattice-Boltzmann simulation. 


\section{Transfer of the Multiphase Field Method}

In this chapter two applications of the multiphase field method are presented, that are more of theoretical interest and not directly related to materials. The first is the construction of generator points for a Laguerre tessellation that recover a certain grain size distribution given by a histogram, which is an interesting mathematical problem $[74,75,76]$. The second application is the utilization of the multiphase field method itself as a load-balancer. Both applications are based on a utilization of the driving force in equation 6.9 in order to manipulate the volume of grains, in order to reach the solution of the specific problem.

\subsection{RVE-Generation with the Multiphase Field Method}

In the following a way of generating representative volume elements (RVE) for polycrystalline materials with a given grain size distribution is presented. The generated RVEs are useful for FEM simulations of the mechanical behavior of polycrystalline materials [75, 76]. 


\subsubsection{Microstructure Generation Using Multiphase Field}

A given grain size distribution in divided into intervals of grain sizes diameters $\left[d_{i-\frac{1}{2}}, d_{i+\frac{1}{2}}\right]$ with a volume fraction $P_{i}$. As the number of grains that fit into one of these intervals is a natural number, we cannot expect to find a microstructure within a limited domain that perfectly agrees with the given grain size distribution. Adding or subtracting a single grain that lies within the interval of the largest grain size, would have the most significant difference in the volume fraction compared to the other intervals. Therefore, the interval with the largest grain size is used to determine the size of the computational domain $\Omega=[0, N]^{d} . N$ is then given by

$$
N=\left(\frac{N_{V_{\max }} V_{\max }}{P_{V_{\max }}}\right)^{1 / d},
$$

with the number of dimensions $d$, the maximum grain size $V_{\max }$, the volume fraction $P_{V_{\max }}$ of grains of size $V_{\max }$ and the number $N_{V_{\max }}$ of grains of size $V_{\max }$ in the domain $\Omega$. With higher $N_{V_{\max }}$ we can also expect to better reproduce the volume fractions of the smaller grain sizes as the as the relative contribution for each individual grain to the total domain volume decreases.

For each grain a pressure

$$
P_{\alpha}:=V_{\alpha}-V_{\alpha}^{G S D}
$$

is computed that describes the volume error compared to its target volume $V_{\alpha}^{G S D}$. Using this pressure a driving fore is introduced that drives the grains towards their target volume. This driving force is given by

$$
\Delta g_{\alpha \beta}:=-q^{d} \frac{P_{\alpha}-P_{\beta}}{S_{\alpha \beta}},
$$

with the number of surface points $S_{\alpha \beta}$ between the grains $\alpha$ and $\beta$, a parameter $q$ and the number of dimensions $d$. The parameter $q$ controls how large influence of the volume error on the evolution of the phases compared to the interface energy. If $q$ is set to small number the interface energy will dominate and a reduction of the volume error will take a long time. If $q$ however is set to a high number the volume error will converge almost instantly. Afterwards the microstructure has time to reduce the interface energy while the volume error is kept on the same level. As 
OpenPhase rescales large driving forces using a tanh-function to maintain the phase field profile. Therefore a high number for $q$ can be selected as it has no physical meaning. Special care needs to be taken so that small grains do not disappear. This is achieved by adding a penalty $p_{\alpha}=-q V_{\min }$ to the pressure $P_{\alpha}$ when $V_{\alpha}<0.5 V_{\min }$, where $V_{\min }$ is the smallest grain size in grain size distribution.

\subsubsection{Inverse Laguerre Tessellation}

The Laguerre Tessellation (also known as Radical Voronoi tessellation or Power Diagram) is a generalization of the Voronoi tessellation that uses weighted seed points $\left(x, r_{x}\right) \in \mathbb{R}^{d} \times \mathbb{R}$. The power of a point $p \in \mathbb{R}^{d}$ to the seed point $\left(x, r_{x}\right)$ is defined as

$$
\operatorname{pow}\left(p,\left(x, r_{x}\right)\right):=\|p-x\|^{2}-r_{x}^{2} .
$$

For a set $S=\left\{\left(x_{1}, r_{1}\right), \ldots,\left(x_{n}, r_{n}\right)\right\}$ the Laguerre tessellation divides a domain $\Omega \subset$ $\mathbb{R}^{d}$ into cells $C_{i}$ with

$$
C_{i}=\left\{y \in \Omega \mid \operatorname{pow}\left(y,\left(x_{i}, r_{i}\right)\right) \leq \operatorname{pow}\left(y,\left(x_{j}, r_{j}\right)\right) \forall j \neq i\right\} .
$$

Therefore any point $p$ on a facet between two Laguerre cells with seed points $x_{\alpha}$ and $x_{\beta}$ and radius $r_{\alpha}$ and $r_{\beta}$ fulfills

$$
\operatorname{pow}\left(p,\left(x_{\alpha}, r_{\alpha}\right)\right)=\operatorname{pow}\left(p,\left(x_{\beta}, r_{\beta}\right)\right) .
$$

Microstructures of polycrystalline materials are generally not Laguerre tessellations and using a solver for the Laguerre inverse problem (LIP) as in [77] will not necessarily provide a good result. However one can try to find a Laguerre tessellation that approximates a certain microstructure as in [78] and [79]. In the following a very simple way to solve the Laguerre approximation problem (LAP) for results of phase field simulation using simulated annealing [80] . Let $S$ be a set of Laguerre generator points and $P$ a set of phase fields that describe a polycrystalline microstructure with $|S|=|P|$. For points $p_{\alpha \beta}$ on the contour line $C_{\alpha \beta}$ at $\phi_{\alpha}=\phi_{\beta}=0.5$ between to grains $\alpha$ and $\beta$ and Laguerre generator points $\left(x_{\alpha}, r_{\alpha}\right) \in S$ and $\left(x_{\beta}, r_{\beta}\right) \in S$ of the according cells we can define a local defect

$$
E\left(p_{\alpha \beta},\left(x_{\alpha}, r_{\alpha}\right),\left(x_{\beta}, r_{\beta}\right)\right):=\| \operatorname{pow}\left(p_{\alpha \beta},\left(x_{\alpha}, r_{\alpha}\right)-\operatorname{pow}\left(p_{\alpha \beta},\left(x_{\beta}, r_{\beta}\right)\right) \| .\right.
$$


By selecting a discrete subset $P_{\alpha \beta} \subset C_{\alpha \beta}$ we can define a global error as

$$
\begin{aligned}
E(S) & :=\sum_{\alpha} \sum_{\beta>\alpha} \sum_{p_{\alpha \beta} \in P_{\alpha \beta}} E\left(p_{\alpha \beta},\left(x_{\alpha}, r_{\alpha}\right),\left(x_{\beta}, r_{\beta}\right)\right) \\
& =\sum_{\alpha} \sum_{\beta>\alpha} \sum_{p_{\alpha \beta} \in P_{\alpha \beta}} \| \operatorname{pow}\left(p_{\alpha \beta},\left(x_{\alpha}, r_{\alpha}\right)-\operatorname{pow}\left(p_{\alpha \beta},\left(x_{\beta}, r_{\beta}\right)\right) \|\right.
\end{aligned}
$$

Using simulated annealing we try to minimize this error by changing the coordinates and radii of the seed points in $(x, r) \in S$. As an initial guess we use the center of mass of each grain as coordinates and radius

$$
r_{\alpha}=\left(\frac{\Gamma\left(\frac{d}{2}+1\right) V_{\alpha}}{\pi^{\frac{d}{2}}}\right)^{\frac{1}{d}},
$$

where $V_{\alpha}$ is the $d$-dimensional volume of grain $\alpha$ and $\Gamma($.$) the well-known gamma$ function [81]. In each step we create a new configuration $S^{n+1}$ by replacing a single randomly selected seed point $\left(x_{\alpha}^{n}, r_{\alpha}^{n}\right) \in S^{n}$ with a new seed point $\left(x_{\alpha}^{n+1}, r_{\alpha}^{n+1}\right) \in$ $U\left(\left(x_{\alpha}^{n}, r_{\alpha}^{n}\right)\right)$ in a neighborhood $U\left(\left(x_{\alpha}^{n}, r_{\alpha}^{n}\right)\right)$ of $\left(x_{\alpha}^{n}, r_{\alpha}^{n}\right)$.

If $\left.\Delta E^{n+1}=E\left(S^{n+1}\right)-E\left(S^{n}\right)\right)<0$ then the new configuration $S^{n+1}$ will be accepted. Otherwise it will be accepted with a probability

$$
P\left(T, \Delta E^{n+1}\right)=e^{-\frac{\Delta E^{n+1}}{T}}=e^{-\frac{E\left(S^{n+1}\right)-E\left(S^{n}\right)}{T}},
$$

with a temperature $T$. In order to calculate the update $\Delta E^{n+1}=E\left(S^{n+1}\right)-E\left(S^{n}\right)$ it is not necessary to compute $E\left(S^{n+1}\right)$ using equation 7.9 as the energy only changes locally for the interface points $p_{\alpha \beta} \in P_{\alpha \beta}$ on interfaces between the Laguerre cell induced by $\left(x_{\alpha}, r_{\alpha}\right)$ and its neighbors. The update is then given by

$$
\begin{aligned}
\Delta E^{n+1}=\sum_{\beta \neq \alpha} \sum_{p_{\alpha \beta} \in P_{\alpha \beta}} & {\left[\| \operatorname{pow}\left(p_{\alpha \beta},\left(x_{\alpha}^{n+1}, r_{\alpha}^{n+1}\right)-\operatorname{pow}\left(p_{\alpha \beta},\left(x_{\beta}, r_{\beta}\right)\right) \|\right.\right.} \\
& -\| \operatorname{pow}\left(p_{\alpha \beta},\left(x_{\alpha}^{n}, r_{\alpha}^{n}\right)-\operatorname{pow}\left(p_{\alpha \beta},\left(x_{\beta}, r_{\beta}\right)\right) \|\right] .
\end{aligned}
$$

Note that with decreasing temperature $T$ the acceptance probability decreases. In the following simulations the inverse starting temperature $T_{0}^{-1}=0.1$ is used, after $m$ iteration steps the inverse temperature is increased by a factor $q=1.005$. The number of iterations $m$ is chosen as the number of seed points times hundred.

In order to compare results the relative volume error

$$
E_{\mathrm{Vol}}\left(\Phi_{1}, \Phi_{2}\right)=\sum_{\alpha} \frac{\left\|V_{\alpha}^{\Phi_{1}}-V_{\alpha}^{\Phi_{2}}\right\|}{\left\|V_{\alpha}^{\Phi_{1}}\right\|}
$$


is used to observe the difference in the volumes $V_{\alpha}^{\Phi}$ of grain between two configurations. Additionally the point wise error of the dominating phase field

$$
E_{\text {Point }}\left(\Phi_{1}, \Phi_{2}\right)=\int_{\Omega} 1-\delta_{I\left(\Phi_{1}(x)\right) I\left(\Phi_{2}(x)\right)} \mathrm{d} x
$$

is introduced with the index function $I(\Phi(x))$, which selects the index $\alpha$ with the maximum phase field value $\phi_{\alpha}(x) \Phi(x)$ in point $x \in \Omega$ and the Kronecker delta. The discretized version of the point wise error

$$
E_{\text {Point }}^{h}\left(\Phi_{1}, \Phi_{2}\right)=\sum_{x_{h} \in \Omega_{h}}\left[1-\delta_{I\left(\Phi_{1}\left(x_{h}\right)\right) I\left(\Phi_{2}\left(x_{h}\right)\right)}\right],
$$

counts the number of grid points in which the index of the dominant phase field differs. In order to account for the size of the domain the relative point wise error

$$
\hat{E}_{\text {Point }}^{h}\left(\Phi_{1}, \Phi_{2}\right)=\frac{1}{\left\|\Omega_{h}\right\|} \sum_{x_{h} \in \Omega_{h}}\left[1-\delta_{I\left(\Phi_{1}\left(x_{h}\right)\right) I\left(\Phi_{2}\left(x_{h}\right)\right)}\right]
$$

is introduced.

Remark 7.1.1. During the phase field step the microstructure can be shape in way that does not correspond to a Laguerre tessellation. Canonical examples are small grains, which are embedded in the bulk of a larger grain or in the interface between two larger grains. Because of this the domain of the larger grain is not convex and therefore the microstructure does not resemble a Laguerre tessellation. If the number of neighbors of a grain is below three in two dimensions or below four in three dimension, the target volume of that grain is exchanged with that of a neighboring grain.

\subsubsection{Low Contrast Examples}

At first we take a look at examples with low contrast between grain sizes. The following volume fractions are used

\begin{tabular}{lllllll} 
Diameter $\left(10^{-6} \mathrm{~m}\right)$ & 10 & 12 & 14 & 16 & 18 & 20 \\
\hline Volume fraction (a) & 30 & 10 & 10 & 10 & 10 & 30 \\
Volume fraction (b) & 10 & 10 & 30 & 30 & 10 & 10
\end{tabular}




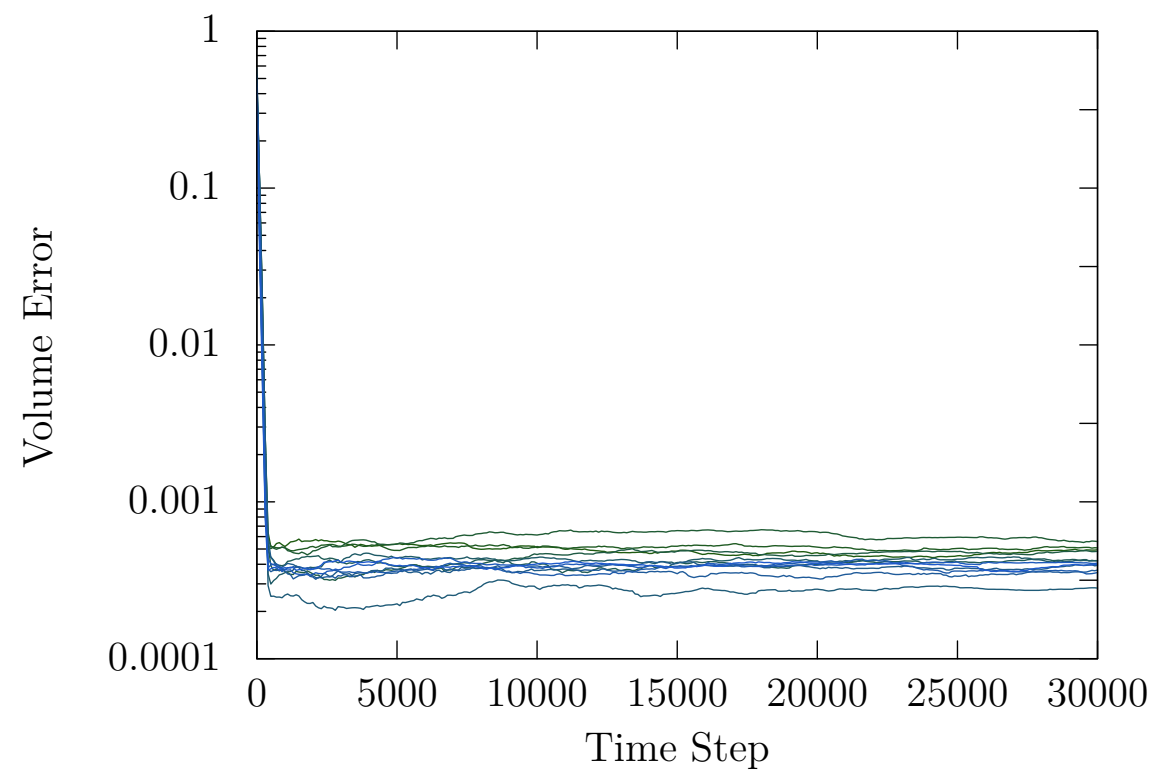

Figure 7.1: Evolution of the volume error for different runs, in which the grain sizes are reassigned randomly. Note that the volume error reaches a plateau nearly instantly.

Using these values a target volume is assigned to each grain. Multiple runs are conducted, in which the target volumes are randomly reassigned. Figure 7.1 shows the evolution of the total relative volume error, which converges nearly instantly. The evolution of the average interfacial energy per grid point is shown in figure 7.2, which compared to the volume error converges much slower. The reason for this behavior is a large parameter $q$.

Figures 7.3 and 7.4 compare the results for these examples. The targeted grain size distribution is shown in blue. The modified grain size distribution for the domain $\Omega$ is depicted in red. The solution of the phase field method is green and the yellow is the solution of a Laguerre Tessellation using generator points obtained by the Inverse Laguerre Tessellation on the phase field solution. The solution of a Laguerre Tessellation using the center of mass and volume of the grains is depicted in olive. In all cases the generator points obtained by LAP recreate the grain size distribution better than the mass centers. Table 7.1 shows that the LAP generator points are give significantly better results than the center of mass points in all (pseudo) metrics, for both examples in both two and three dimensions. 


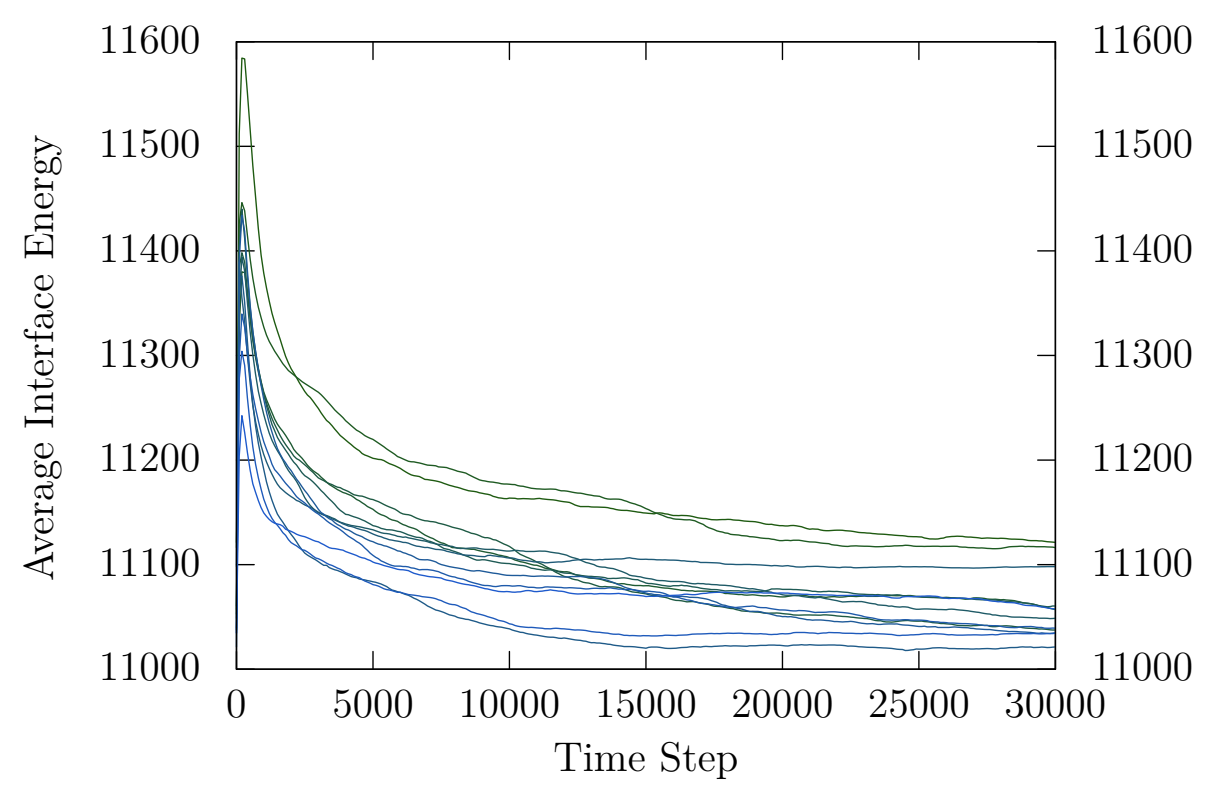

Figure 7.2: Evolution of the average interface energy for different runs, in which the grain sizes are reassigned randomly. Notice that in contrast to the volume error the average interface energy takes more time steps to converge.

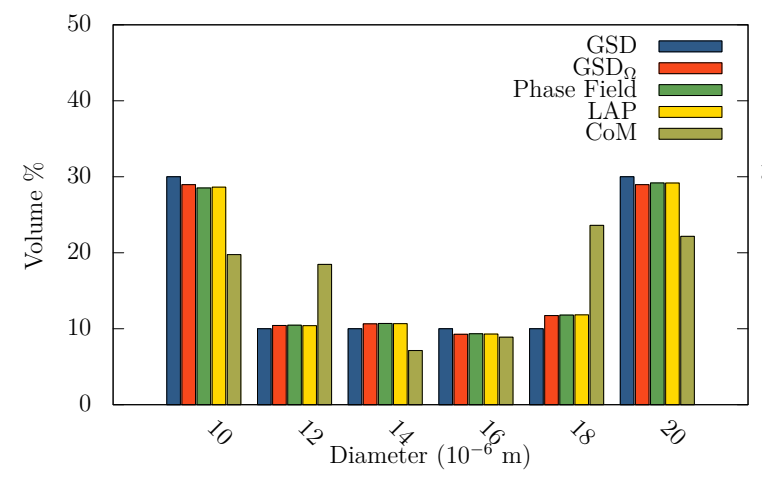

(a) 2D-Simulation.

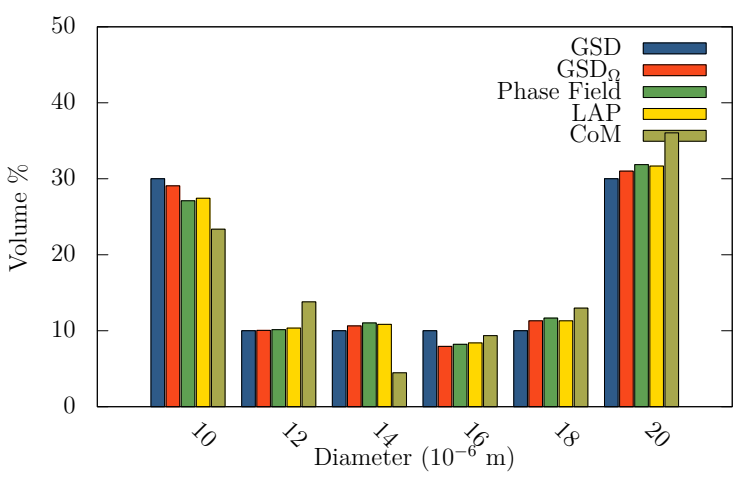

(b) 3D-Simulation.

Figure 7.3: Volume comparison for example (b) with low contrast in grain sizes of section 7.1.3. 


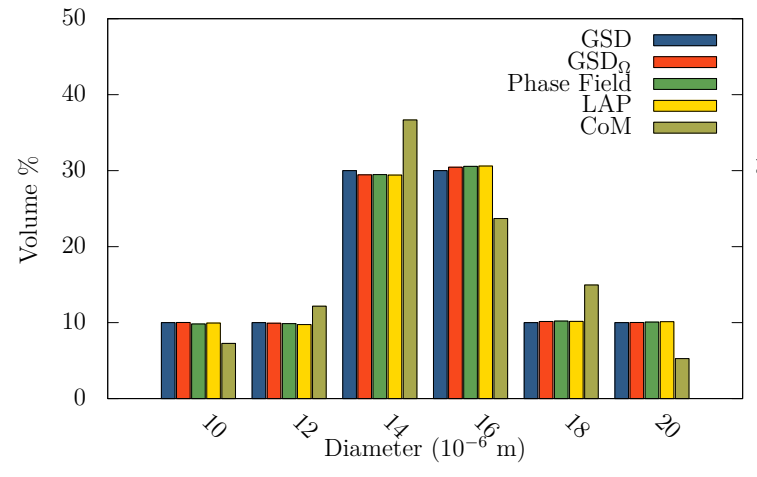

(a) 2D-Simulation.

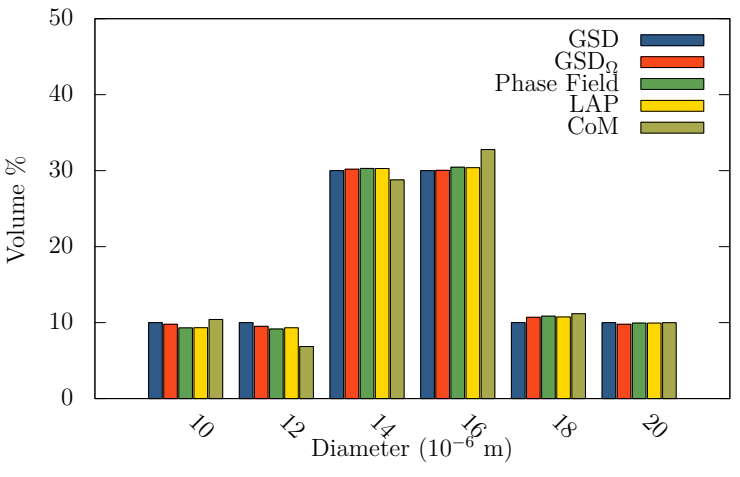

(b) 3D-Simulation.

Figure 7.4: Volume comparison for example (b) with low contrast in grain sizes of section 7.1.3.

$$
\begin{array}{l|r}
E_{\text {Vol }}\left(\Phi, \Phi_{L A P}\right) & 0.0204105 \\
E_{\text {Vol }}\left(\Phi, \Phi_{C O M}\right) & 0.183864 \\
E_{\text {Point }}^{h}\left(\Phi, \Phi_{L A P}\right) & 72 \\
E_{\text {Point }}^{h}\left(\Phi, \Phi_{C O M}\right) & 880 \\
\hat{E}_{\text {Point }}^{h}\left(\Phi, \Phi_{L A P}\right) & 0.0176 \\
\hat{E}_{\text {Point }}^{h}\left(\Phi, \Phi_{C O M}\right) & 0.214
\end{array}
$$

(a) Comparison of the LAP and COM solutions for the grain size distribution in figure 7.3a.

$$
\begin{array}{l|r}
E_{\mathrm{Vol}}\left(\Phi, \Phi_{L A P}\right) & 0.0501826 \\
E_{\mathrm{Vol}}\left(\Phi, \Phi_{C O M}\right) & 0.241994 \\
E_{\text {Point }}^{h}\left(\Phi, \Phi_{L A P}\right) & 1564 \\
E_{\text {Point }}^{h}\left(\Phi, \Phi_{C O M}\right) & 8604 \\
\hat{E}_{\text {Point }}^{h}\left(\Phi, \Phi_{L A P}\right) & 0.0579 \\
\hat{E}_{\text {Point }}^{h}\left(\Phi, \Phi_{C O M}\right) & 0.3187
\end{array}
$$

(c) Comparison of the LAP and COM solutions for the grain size distribution in figure $7.3 \mathrm{~b}$.

$$
\begin{array}{l|r}
E_{\mathrm{Vol}}\left(\Phi, \Phi_{L A P}\right) & 0.01529 \\
E_{\mathrm{Vol}}\left(\Phi, \Phi_{C O M}\right) & 0.218637 \\
E_{\text {Point }}^{h}\left(\Phi, \Phi_{L A P}\right) & 225 \\
E_{\text {Point }}^{h}\left(\Phi, \Phi_{C O M}\right) & 2958 \\
\hat{E}_{\text {Point }}^{h}\left(\Phi, \Phi_{L A P}\right) & 0.01794 \\
\hat{E}_{\text {Point }}^{h}\left(\Phi, \Phi_{C O M}\right) & 0.23581
\end{array}
$$

(b) Comparison of the LAP and COM solutions for the grain size distribution in figure 7.4a.

$$
\begin{array}{l|r}
E_{\mathrm{Vol}}\left(\Phi, \Phi_{L A P}\right) & 0.026208 \\
E_{\mathrm{Vol}}\left(\Phi, \Phi_{C O M}\right) & 0.168909 \\
E_{\text {Point }}^{h}\left(\Phi, \Phi_{L A P}\right) & 3056 \\
E_{\text {Point }}^{h}\left(\Phi, \Phi_{C O M}\right) & 18415 \\
\hat{E}_{\text {Point }}^{h}\left(\Phi, \Phi_{L A P}\right) & 0.03587 \\
\hat{E}_{\text {Point }}^{h}\left(\Phi, \Phi_{C O M}\right) & 0.21618
\end{array}
$$

(d) Comparison of the LAP and COM solutions for the grain size distribution in figure $7.4 \mathrm{~b}$.

Table 7.1: Error comparison for the low contrast example in section 7.1.3. 


\subsubsection{High Contrast Examples}

Now we take a look at examples with a high contrast between grain sizes. The following volume fractions are being used

\begin{tabular}{lllllll} 
Diameter $\left(10^{-6} \mathrm{~m}\right)$ & 10 & 20 & 30 & 40 & 60 & 70 \\
\hline Volume fraction (a) & 30 & 10 & 10 & 10 & 10 & 30 \\
Volume fraction (b) & 10 & 10 & 30 & 30 & 10 & 10
\end{tabular}

As seen in figures 7.5 and 7.6 the examples shows similar results as the low contrast examples. Again the LAP generator points recreate the grain size distribution more accurately than the center of mass points. Table 7.2 confirms that the LAP generator points improve the accuracy significantly. The volume error $E_{\mathrm{Vol}}$ is almost a magnitude smaller with the LAP generator points. The point error $E_{\text {Point }}$ is improved by a factor between two and four. Figure 7.7 shows the microstructure obtained from the phase field method (7.7a) as well as the microstructure obtained from a Laguerre tessellation using the center of mass generator points (7.7b) and generator points obtained from the Laguerre approximation solution using simulated annealing $(7.7 \mathrm{c})$. It can be easily seen that the LAP generator points recreate the shape of the grains more accurately than the center of mass points.

\subsubsection{Conclusions}

Overall it can be seen that the phase field method itself can recreate a given grain size distribution accurately in a simple way. This intermediate solution could be triangulated and used to create a FEM-mesh directly. Often it is easier to use a set of Laguerre generator points in a tool like Neper [82] to generate an FEM mesh. Simply using the center of mass as generator point for a Voronoi or Laguerre tessellation does not reproduce the microstructure accurately enough. It was shown that a simple simulated annealing approach can significantly improve the position 


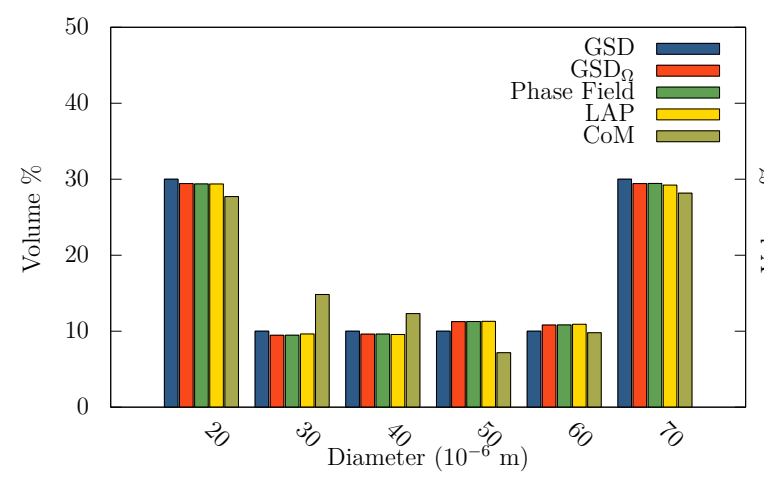

(a) 2D-Simulation.

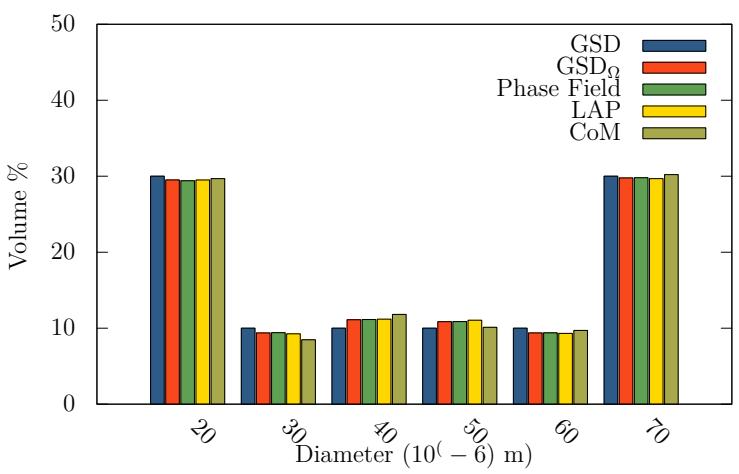

(b) 3D-Simulation.

Figure 7.5: Volume comparison for example (b) with high contrast in grain sizes of section 7.1.4.

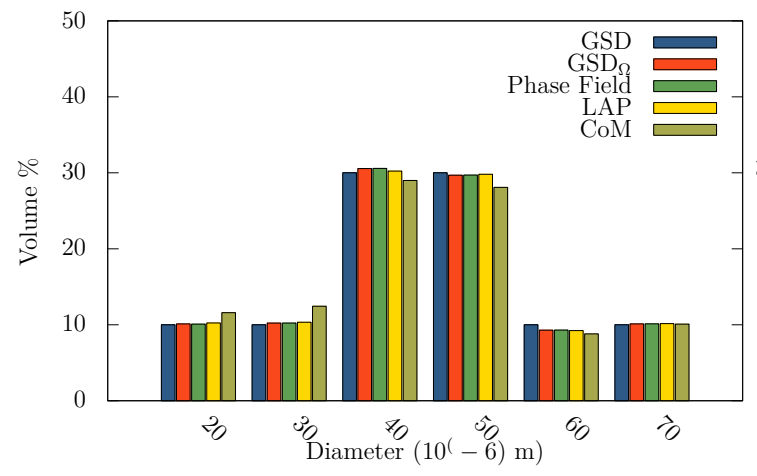

(a) 2D-Simulation.

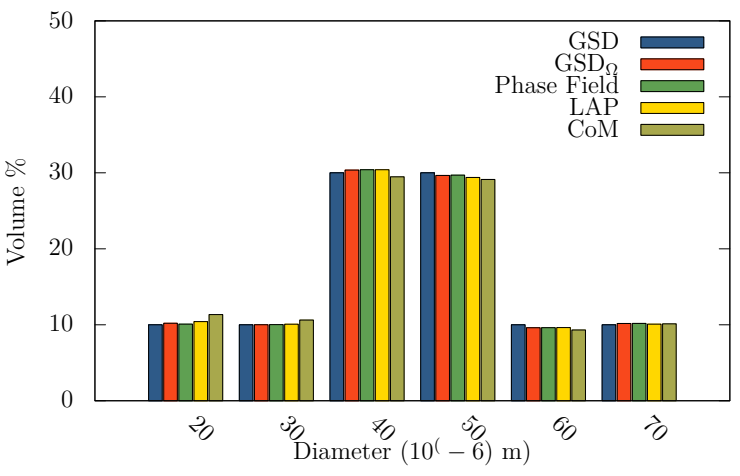

(b) 3D-Simulation.

Figure 7.6: Volume comparison for example (b) with high contrast in grain sizes of section 7.1.4. 


\begin{tabular}{l|r}
$E_{\mathrm{Vol}}\left(\Phi, \Phi_{L A P}\right)$ & 0.0282552 \\
$E_{\mathrm{Vol}}\left(\Phi, \Phi_{C O M}\right)$ & 0.220481 \\
$E_{\text {Point }}^{h}\left(\Phi, \Phi_{L A P}\right)$ & 1976 \\
$E_{\text {Point }}^{h}\left(\Phi, \Phi_{C O M}\right)$ & 8133 \\
$\hat{E}_{\text {Point }}^{h}\left(\Phi, \Phi_{L A P}\right)$ & 0.0383 \\
$\hat{E}_{\text {Point }}^{h}\left(\Phi, \Phi_{C O M}\right)$ & 0.1578
\end{tabular}

(a) Comparison of the LAP and COM solutions for the grain size distribution in figure $7.5 \mathrm{a}$.

\begin{tabular}{l|r}
$E_{\mathrm{Vol}}\left(\Phi, \Phi_{L A P}\right)$ & 0.038943 \\
$E_{\mathrm{Vol}}\left(\Phi, \Phi_{C O M}\right)$ & 0.311148 \\
$E_{\text {Point }}^{h}\left(\Phi, \Phi_{L A P}\right)$ & 65463 \\
$E_{\text {Point }}^{h}\left(\Phi, \Phi_{C O M}\right)$ & 287675 \\
$\hat{E}_{\text {Point }}^{h}\left(\Phi, \Phi_{L A P}\right)$ & 0.055 \\
$\hat{E}_{\text {Point }}^{h}\left(\Phi, \Phi_{C O M}\right)$ & 0.242
\end{tabular}

(c) Comparison of the LAP and COM solutions for the grain size distribution in figure $7.5 \mathrm{~b}$.

$$
\begin{array}{l|r}
E_{\mathrm{Vol}}\left(\Phi, \Phi_{L A P}\right) & 0.0361299 \\
E_{\mathrm{Vol}}\left(\Phi, \Phi_{C O M}\right) & 0.168035 \\
E_{\text {Point }}^{h}\left(\Phi, \Phi_{L A P}\right) & 6767 \\
E_{\text {Point }}^{h}\left(\Phi, \Phi_{C O M}\right) & 22620 \\
\hat{E}_{\text {Point }}^{h}\left(\Phi, \Phi_{L A P}\right) & 0.044 \\
\hat{E}_{\text {Point }}^{h}\left(\Phi, \Phi_{C O M}\right) & 0.1472
\end{array}
$$

(b) Comparison of the LAP and COM solutions for the grain size distribution in figure 7.6a.

$$
\begin{array}{l|r}
E_{\mathrm{Vol}}\left(\Phi, \Phi_{L A P}\right) & 0.0362062 \\
E_{\mathrm{Vol}}\left(\Phi, \Phi_{C O M}\right) & 0.147697 \\
E_{\text {Point }}^{h}\left(\Phi, \Phi_{L A P}\right) & 176392 \\
E_{\text {Point }}^{h}\left(\Phi, \Phi_{C O M}\right) & 341653 \\
\hat{E}_{\text {Point }}^{h}\left(\Phi, \Phi_{L A P}\right) & 0.0493 \\
\hat{E}_{\text {Point }}^{h}\left(\Phi, \Phi_{C O M}\right) & 0.0953
\end{array}
$$

(d) Comparison of the LAP and COM solutions for the grain size distribution in figure $7.6 \mathrm{~b}$.

Table 7.2: Error comparison for the high contrast example in section 7.1.4.

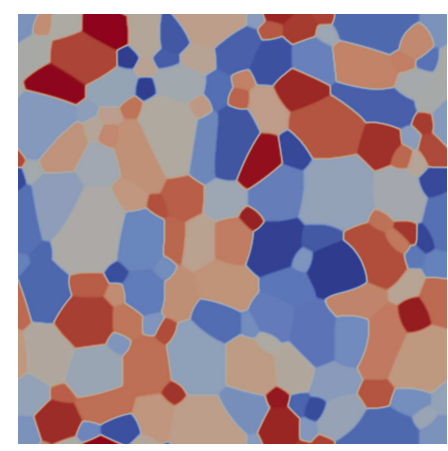

(a) Phase field

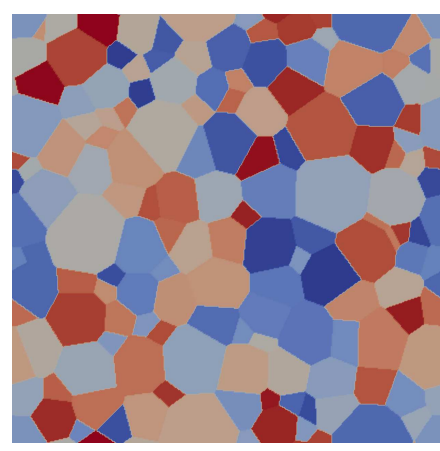

(b) CoM generator points

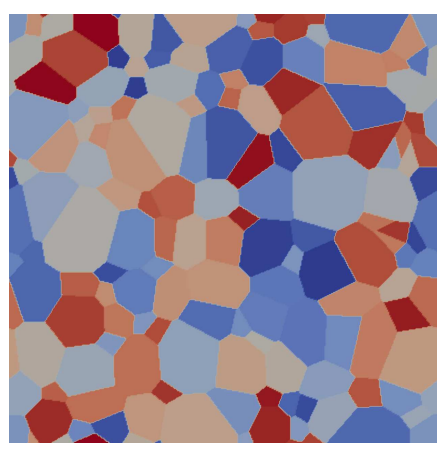

(c) LAP generator points

Figure 7.7: Microstructures obtain from the different method for the example in section 7.1.4. 
and radius of the Laguerre generator points, so that the microstructure is reproduced accurately. This allows a two step approach to generate a set of Laguerre seed points that recreate a given grain size distribution, first phase field to generate a microstructure similar to a Laguerre tessellation, then simulated annealing to obtain the seed points of this tessellation. 


\subsection{Load-balancing with the Multiphase Field Method}

In the following an alternative approach to load-balancing based on the multiphase field method will be presented, that can be used for a variety of applications, such as the phase field method as well as cell-based particle methods such as Molecular Dynamics [83] or Smoothed Particle Hydrodynamics [84].

\subsubsection{Implementation of the Multiphase-Load-balancer}

As in chapter 5 domain-decomposition is used a general approach. This time, however, we do not use a domain over-decomposition, that means each process only handles a single sub-domain and no graph-partitioning is needed in order to assign the sub-domains (or blocks) to processes. The sub-domains $\Omega_{p}$ form a cover of the domain $\Omega \subseteq \cup_{p \in P} \Omega_{p}$. Each sub-domain $\Omega_{p}$ is located on one process $p$ and is associated with a phase field $\phi_{p}$. The wide halo approach as described in section 5.1.1 is utilized again to reduce the frequency of communications within a time step. Let $\Omega_{\phi_{p}}$ be the set of grid points for which $\phi_{p}$ is the majority phase, i.e. $\phi_{p} \geq \phi_{\alpha}$ for all $\alpha \neq p$. If two or more phases are tied the highest phase index is chosen. The subdomain $\Omega_{p}$ is the minimal rectangular domain that contains all these grid points, i.e. $\Omega_{\phi_{p}} \subset \Omega_{p}$.

The computational load $\omega_{p}$ on each process $p \in P$ is used to determine the evolution of the phase fields by introducing a driving force

$$
\Delta g_{\alpha \beta}:=-q^{d} \frac{\omega_{\alpha}-\omega_{\beta}}{S_{\alpha \beta}},
$$

with the surface area $S_{\alpha \beta}$ between the phase fields $\phi_{\alpha}$ and $\phi_{\beta}$. This driving force moves the interface between phases towards the phase with a higher associated computational load $\omega_{q}$. Thus reducing the size of $\Omega_{\phi_{q}}$. If the number of grid points in the set $\Omega_{\phi_{q}}$ correlates with the computational load $\omega_{q}$, then this method can be 
used for load-balancing.

The sub-domains $\Omega_{p}$ are the minimal rectangular boxes aligned with the coordinate axes that contain all grid points with $\phi_{p}>0$ as depicted in figure 7.8. Note that the internal grid points can also overlap. The phase field method is applied in these grid points and the wide halo as described in section 5.1.1. In order to update data in the wide halo the overlap with the internal grid points of other processes is calculated and the communication is conducted in the same way as mentioned in section 5.1.2.

In the following sections two cases will be examined. The first will be the application of the multiphase field load-balancing on the multiphase field method itself. Then a cell-based molecular dynamics implementation will be load-balanced by the multiphase field load-balancer. Each cell will be associated with a grid point in the multiphase field load-balancer.

Significant advantages of this load-balancing scheme compared to the one presented in chapter 5 is avoiding the migration of data and the natural reduction of interfaces between processes and consequently a reduction of the communication costs. A significant downside is the requirement for a large number of load-balancing steps in order to find a suitable domain decomposition.

A load-imbalance tolerance is introduced in order to save the cost of calculating the updates of the load-balancing phase fields as well as avoiding the growth of domains with lower computational load without improving the overall performance.

\subsubsection{Load-balancing Multiphase Field Simulations}

An interesting example is the utilization of the multiphase field load-balancer on the multiphase field method itself. Of course this only makes sense if the computational costs for the multiphase field method of the load-balancer are smaller than those of the simulated system. Therefore the normal grain growth simulation can only benefit from this load-balancing approach as long as the number of different phase fields is sufficiently large and the interfaces between grains are much denser than 


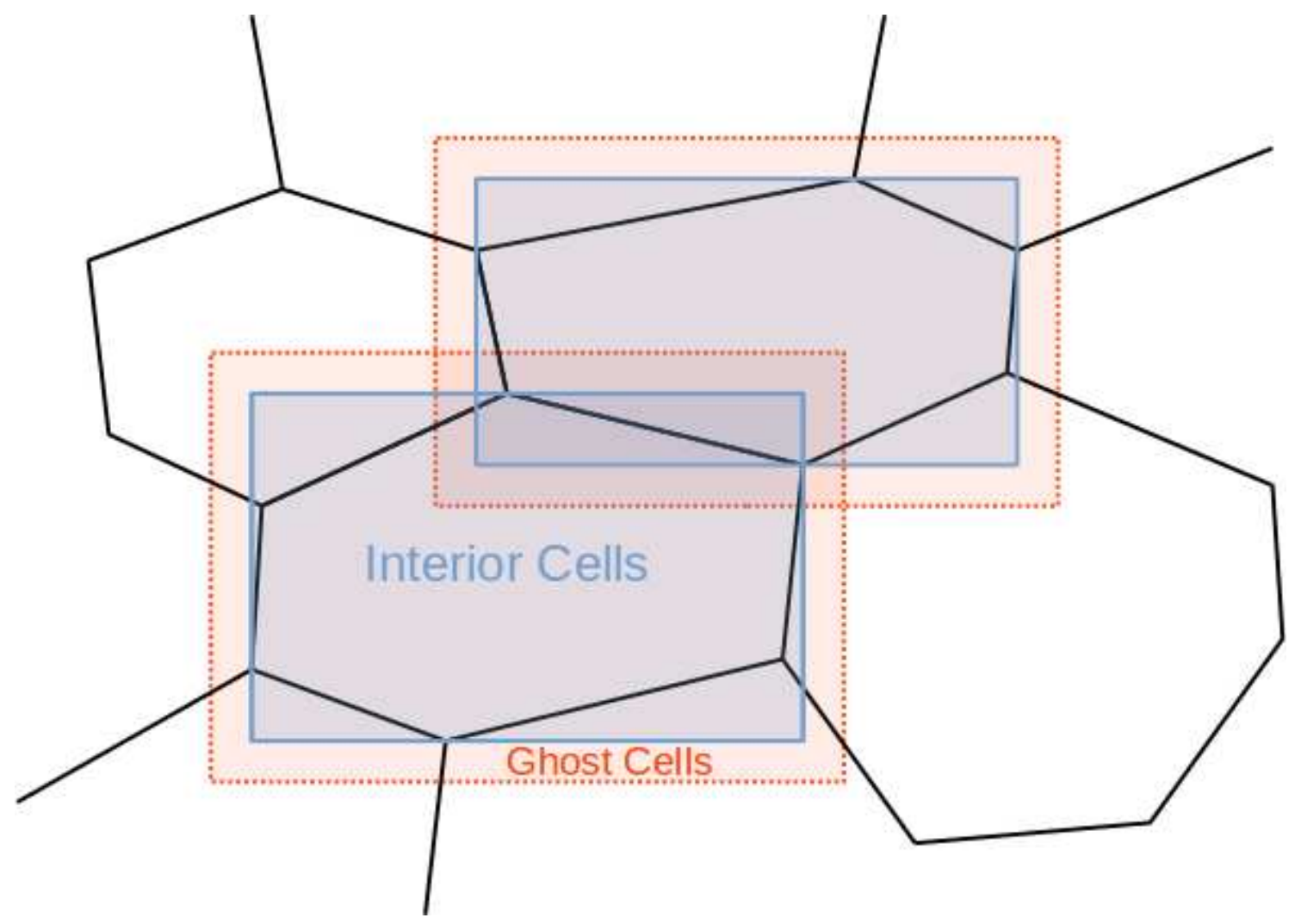

Figure 7.8: Depiction of the way the multiphase field load-balancer decomposes the domain into overlapping sub-domain. Note that interior points can also overlap.

the interfaces between the load-balancing phase fields.

As the load-balancer applies the same driving force in all grid points in the interface between two phase fields, the computationally expensive driving force averaging step can be omitted. Simulations that need to calculate and average the driving force are therefore more likely to benefit from this load-balancing approach. Subsequently the $\mathrm{Mg}-\mathrm{Al}$ alloy solidification benchmark from section 6.5.2 is repeated with the multiphase load-balancer.

The wall time per time step is shown in figures 7.9 and 7.10 over time for 24 MPIprocesses with load-balancing tolerances of $20 \%$ and $5 \%$. In both examples the load-balancer reaches a plateau after 500 time steps, after which the maximum load of the processes is not reduced any more. In both cases the maximum load is on the same level. With a tolerance of $5 \%$ the cost of a time step is almost the same for all processes, while with a $20 \%$ tolerance the individual loads of the processes 
differ significantly. Figures 7.11 and 7.12 show the respective domain sizes for each process in the number of grid points belonging to each process. With a $5 \%$ tolerance the size of the largest domain is about $20 \%$ larger, while the sizes of the smallest domains are about the same. The domains, which end up as the smallest domains, have the most computational load per grid point and are the most heavily loaded domains in the beginning. As they shrink the load-balance and consequently the wall time per step improves.

Figures 7.13 and 7.14 show the scaled up simulation with 96 MPI-processes. The same behavior as above can be observed, although in this case it takes slightly longer to reach a plateau. Note that the plots only show the actual time spend in the multiphase field calculations and does not take into account the time spend in load-balancing and communication. Adding those contributions the following average times after reaching the plateau were obtained

\begin{tabular}{lll|r} 
Cores & Threads & Tolerance & Time (seconds) \\
\hline 24 & 1 & 1.2 & 2.2832 \\
24 & 1 & 1.05 & 2.4109 \\
24 & 4 & 1.2 & 1.2875 \\
96 & 1 & 1.2 & 3.5992 \\
96 & 1 & 1.05 & 3.6990 \\
96 & 4 & 1.2 & 2.0070 \\
96 & 4 & 1.05 & 2.1399 \\
384 & 4 & 1.2 & 3.0128
\end{tabular}

For 24 processes the wall time is about the same as for the benchmark in section 6.5.2. The larger simulation using only 1 threads however is significantly slower. Using 4 threads however gives a comparable result. The higher tolerance of $20 \%$ gives a better overall performance in all tests. As the reduced tolerance does not decrease the maximum load as seen in figures 7.10, 7.14, 7.9 and 7.13, an improved performance could not be expected. However, due to the reduced variance in the computational load, all communication happens at the same time and could increase contention of the network, which could explain the slight performance 


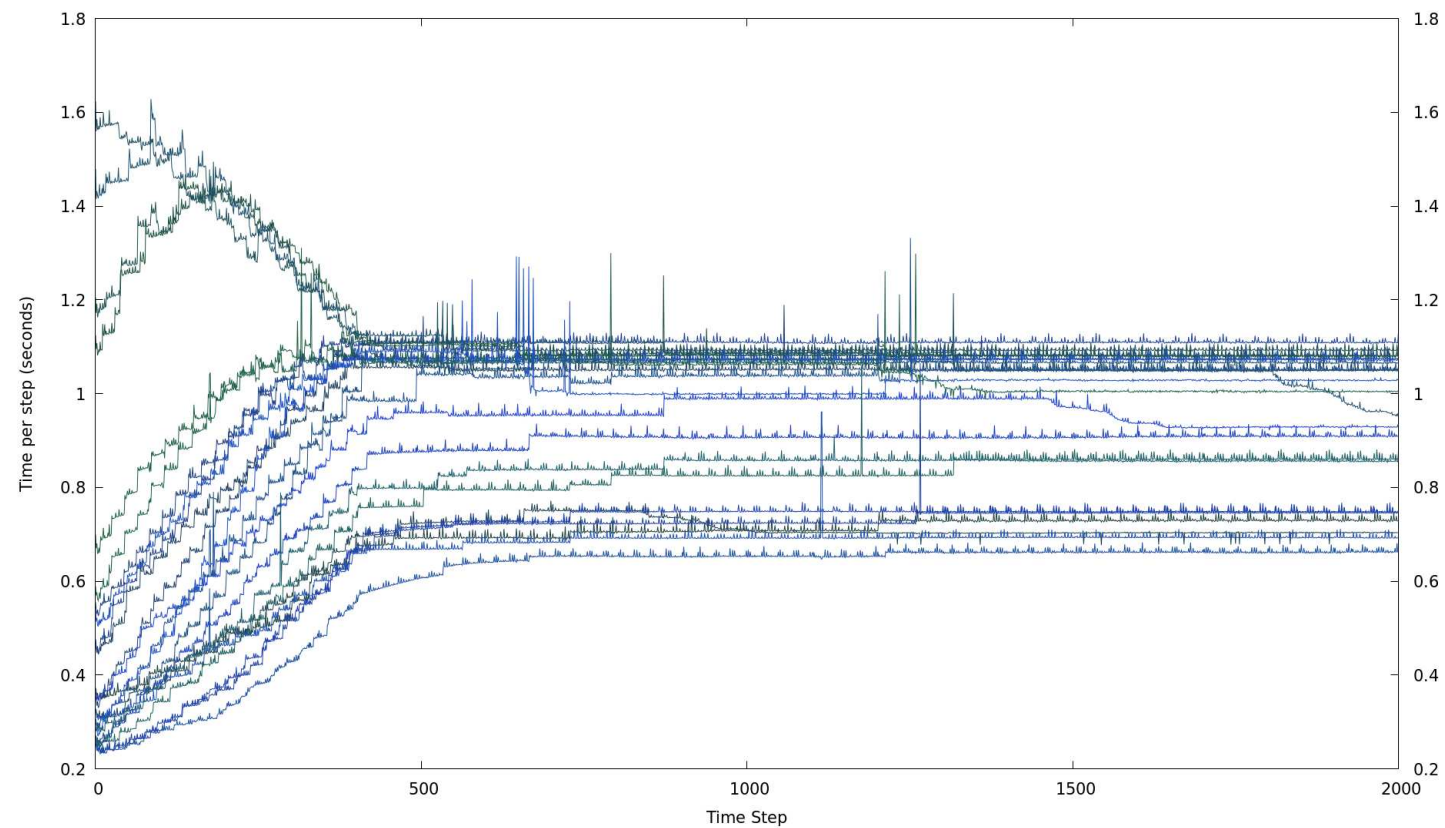

Figure 7.9: Time per step for each process overtime with 24 MPI-processes and a loadbalancing tolerance of $20 \%$.

loss. The largest benchmark using 384 cores with 96 MPI-processes and 4 OpenMP threads is significantly slower than the results for 4 threads given in section 6.5.2. Overall this parallelization does not scale as well as the load-balancing presented in chapter 5 .

A significant downside of the current implementation of this load-balancing technique in conjunction with the phase field method, however, is the overhead created by overlapping domains as depicted in figure 7.8. The calculations are always executed in all grid points contained in a process's domain and not limited to the overlap with its corresponding load-balancing phase field. The overlap of internal grid points, which was avoided in the parallelization presented in chapter 5, can cause significant additional work. Using a hybrid parallelization reduces the negative effect of the overlap, but for large simulations it cannot be avoided. In order to avoid this behavior significant changes to the OpenPhase library or completely new multiphase code would be required. In order to test the multiphase field load-balancer for a problem, in which calculations on process $\mathrm{p}$ are skipped in grid points with $\phi_{p}=0$, it will be applied to cell-based Molecular Dynamics simulations in section 7.2.3. 


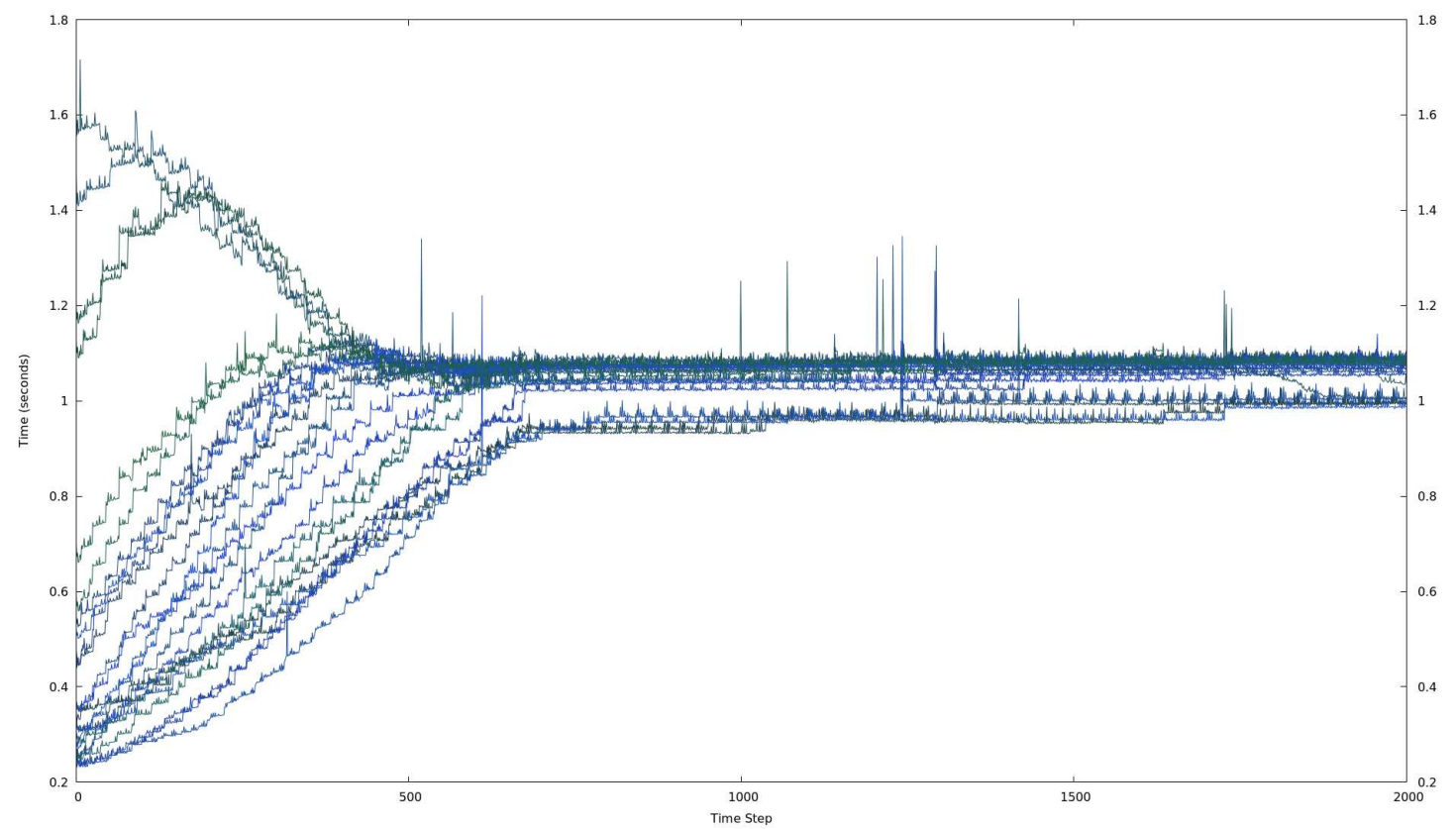

Figure 7.10: Time per step for each process overtime with 24 MPI-processes and a loadbalancing tolerance of $5 \%$.

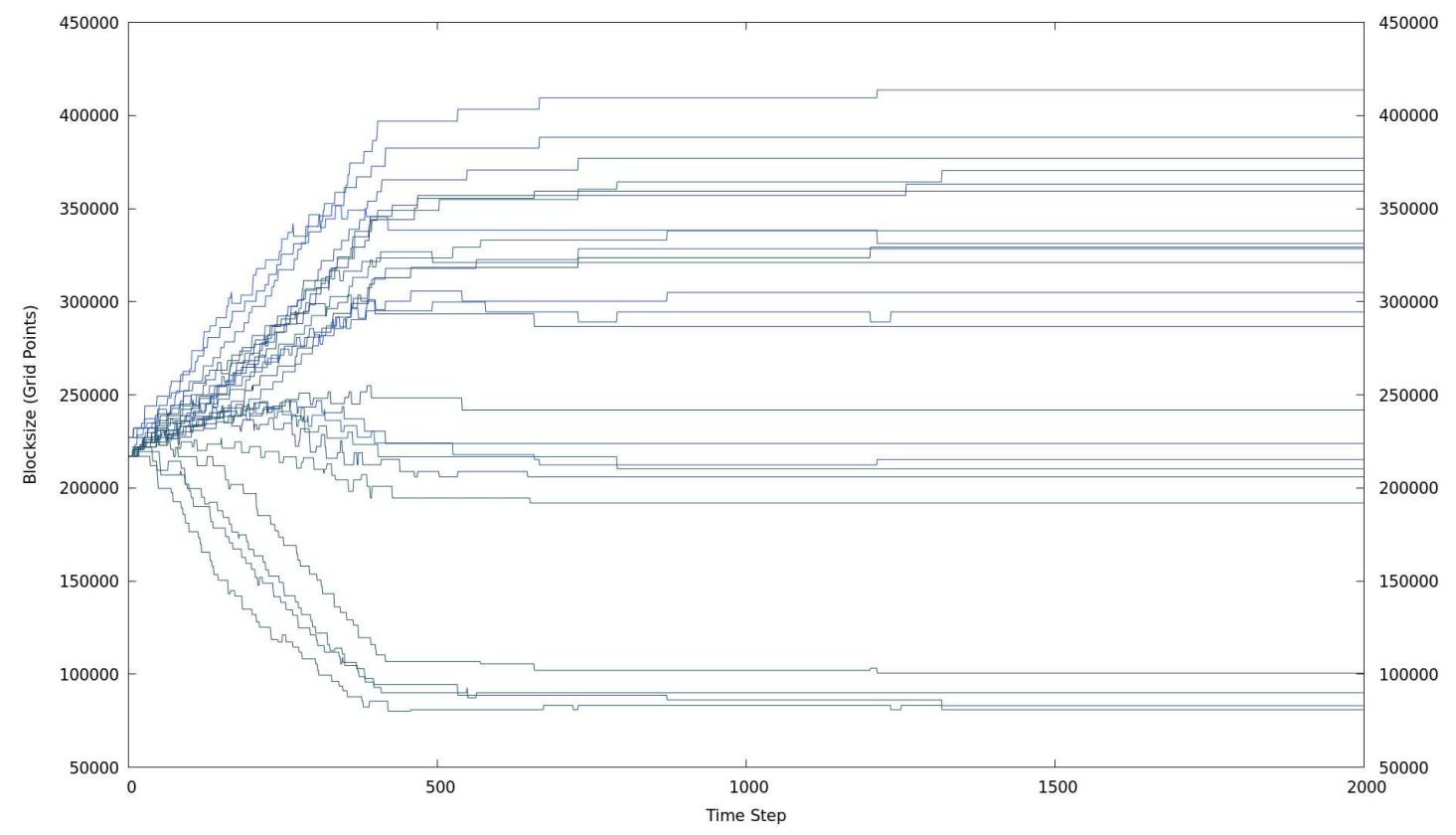

Figure 7.11: Number of grid points for each process overtime with 24 MPI-processes and a load-balancing tolerance of $20 \%$. 


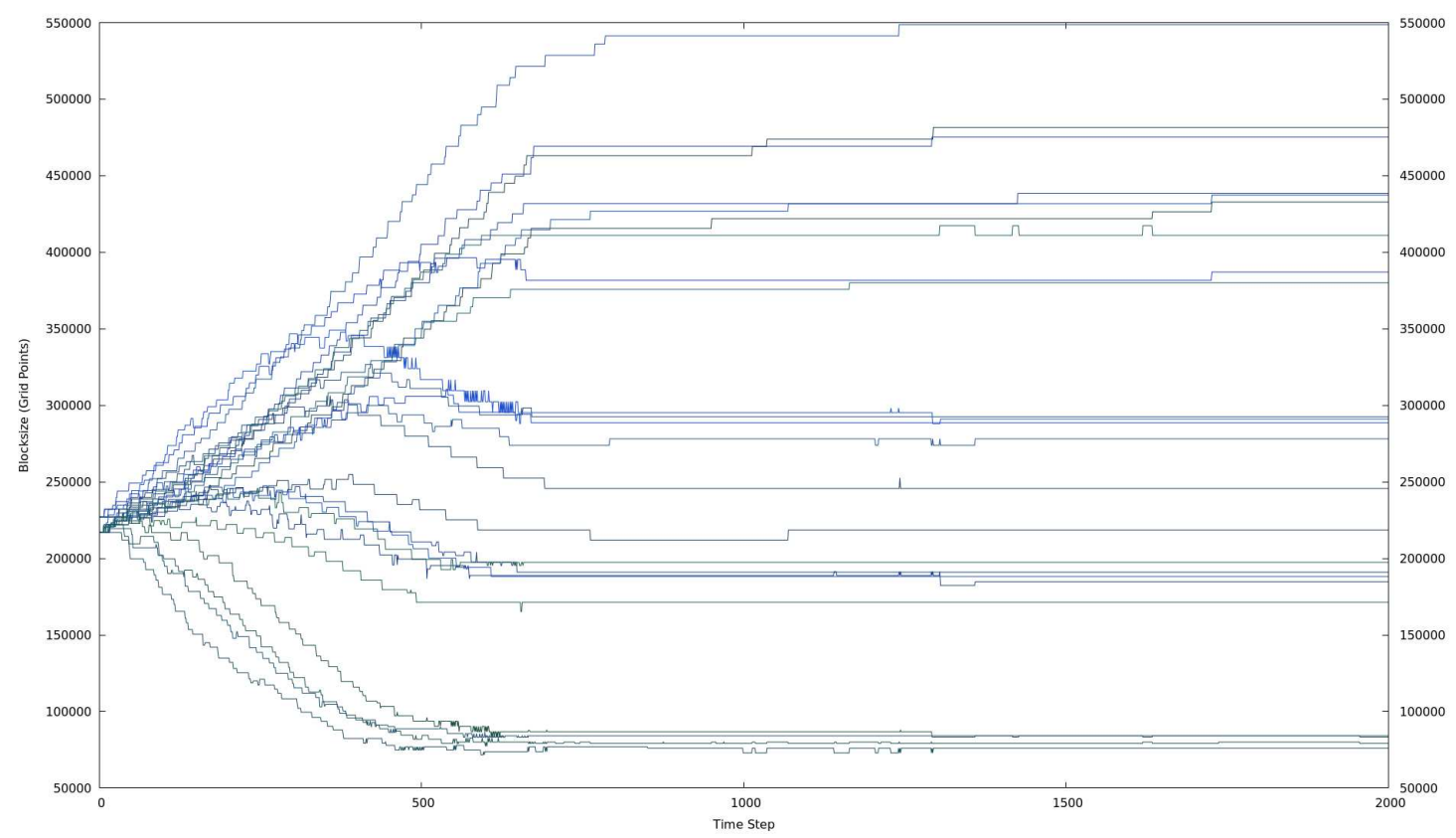

Figure 7.12: Number of grid points for each process overtime with 24 MPI-processes and a load-balancing tolerance of $5 \%$.

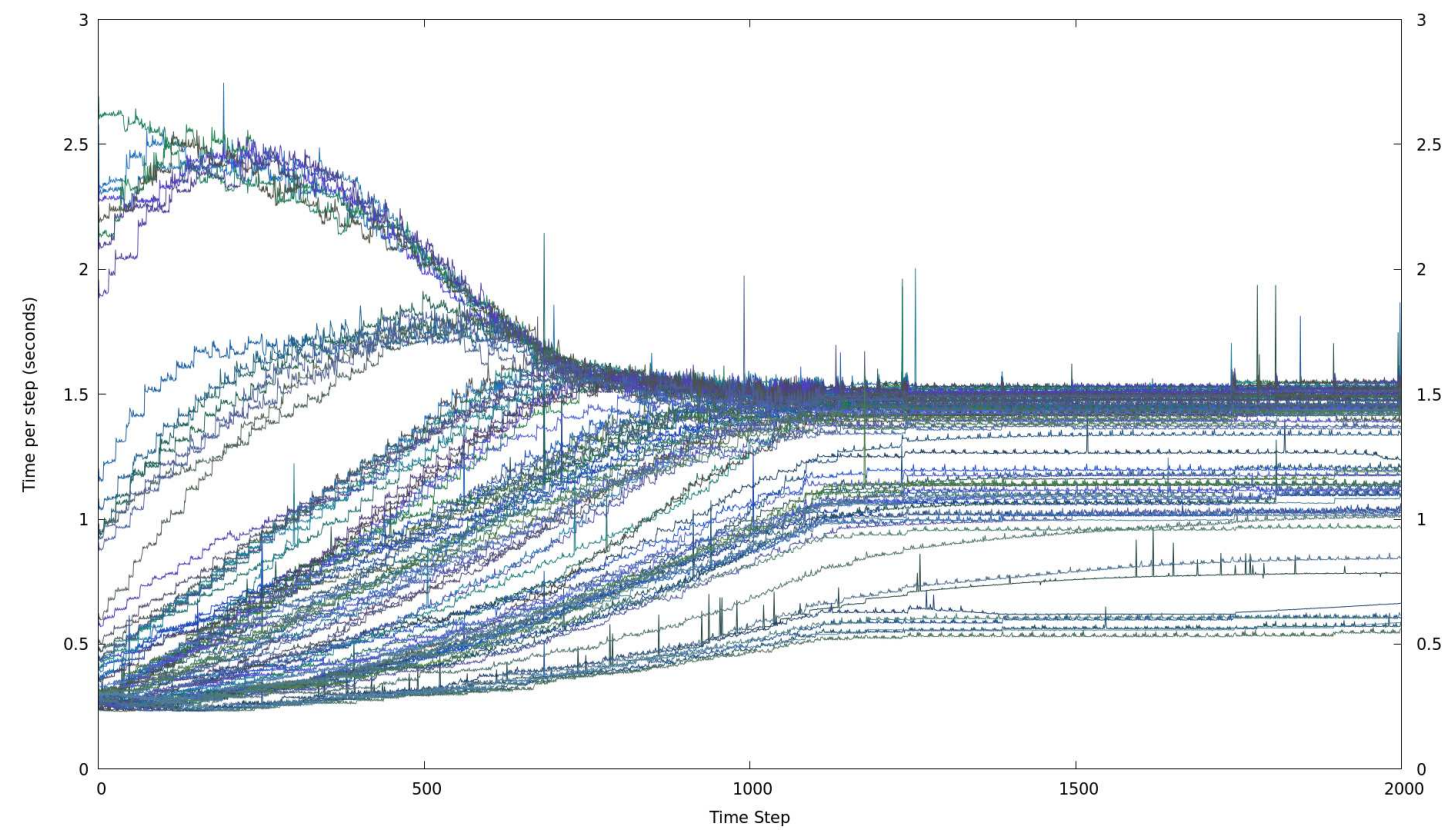

Figure 7.13: Time per step for each process overtime with 96 MPI-processes and a loadbalancing tolerance of $20 \%$. 


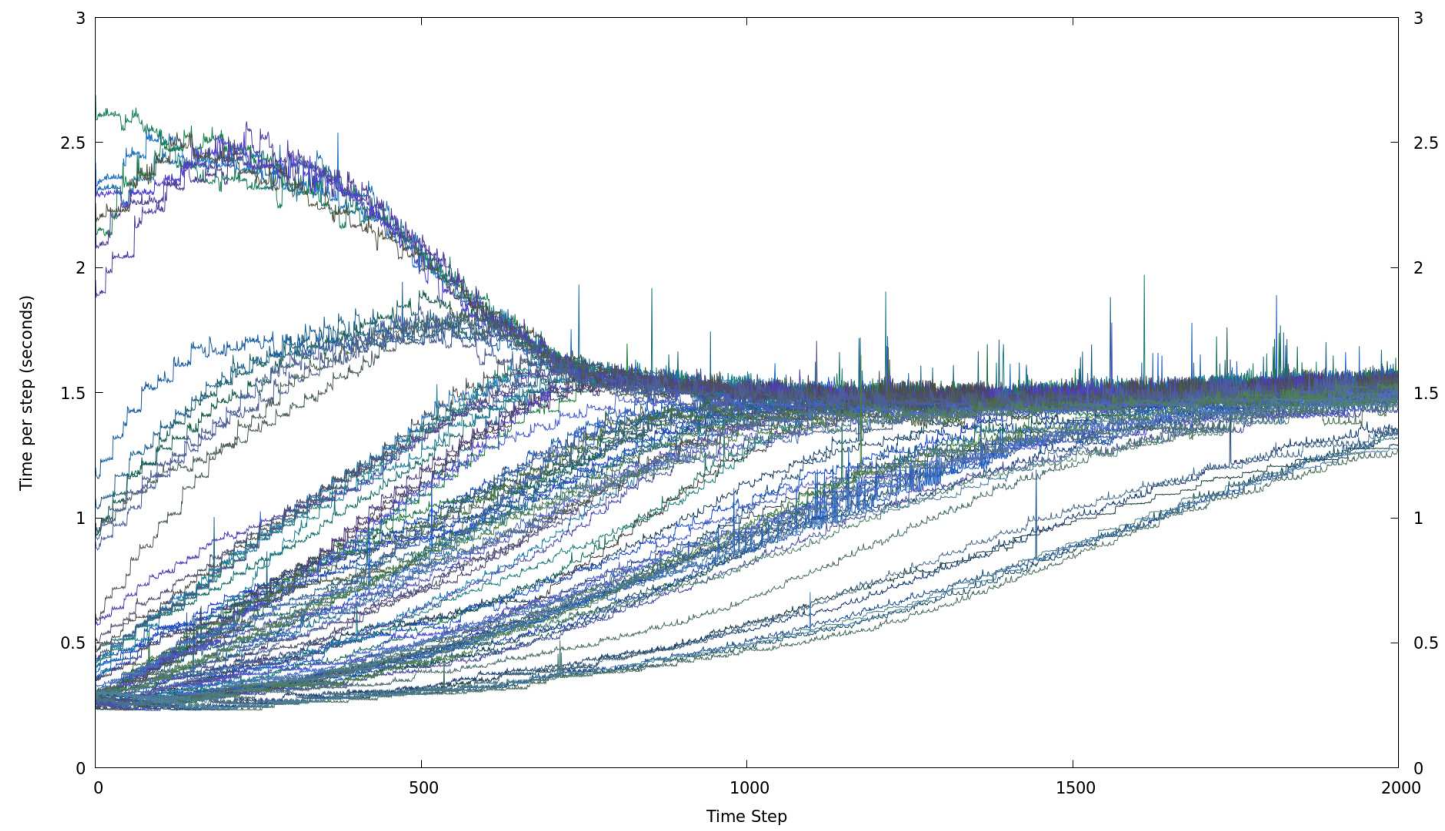

Figure 7.14: Time per step for each process overtime with 96 MPI-processes and a loadbalancing tolerance of $5 \%$.

\subsubsection{Load-balancing MD-Simulations}

As second example the multiphase load-balancer is used to adaptively load-balance a simple cell-based particle method. The method of molecular dynamics is an Nbody simulation, that computes the movement of atoms [83]. It iteratively solves the classical equations of motion

$$
\begin{aligned}
m_{i} \ddot{r}_{i} & =f_{i}, \\
f_{i} & =-\frac{\partial}{\partial r_{i}} U(R),
\end{aligned}
$$

for particles with position $r_{i}$, mass $m_{i}$. The force $f_{i}$ is derived from a potential energy $U(R)$ depending on the set of particles $R$ in the system.

A commonly used potential is the simple Lennard-Jones potential depicted in figure 


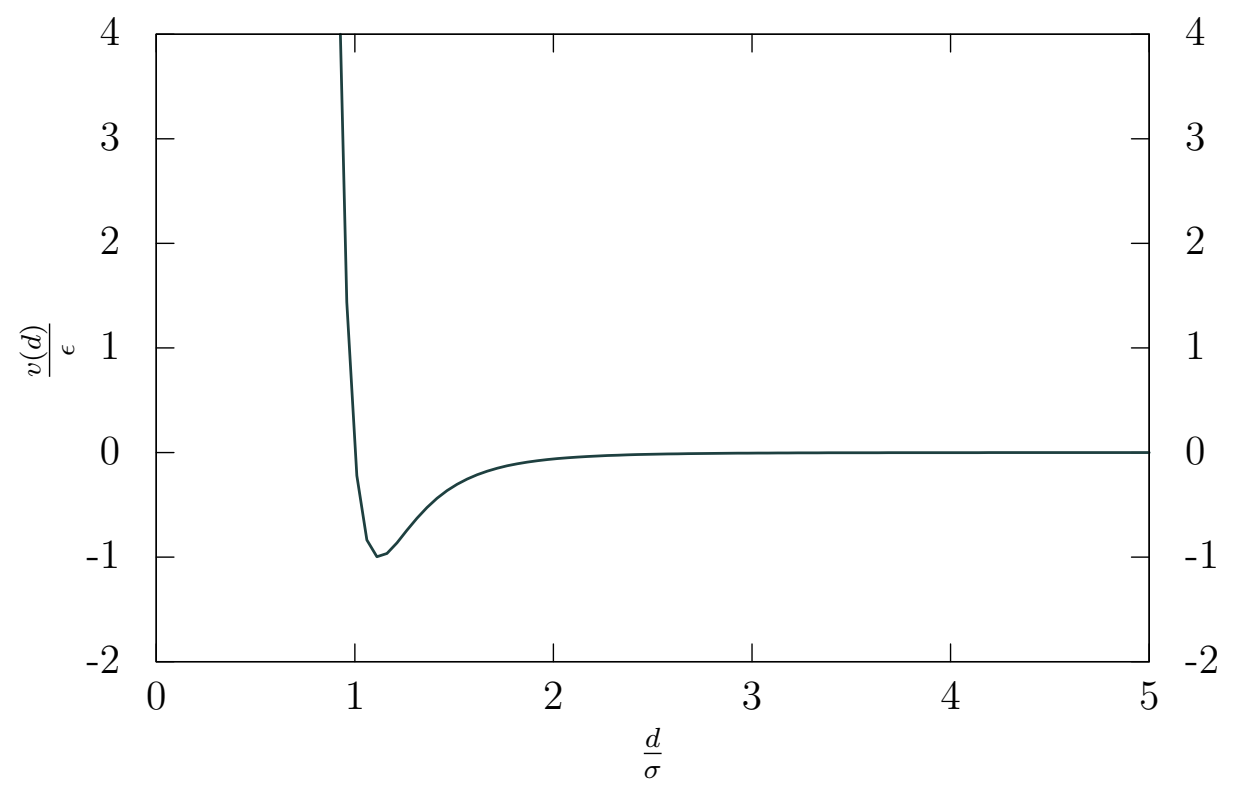

Figure 7.15: The Lennard-Jones potential

7.15

$$
\begin{aligned}
U(R) & =\sum_{i} \sum_{j>i} v\left(r_{i}, r_{j}\right), \\
v\left(r_{i}, r_{j}\right)=v\left(d\left(r_{i}, r_{j}\right)\right) & =4 \epsilon\left[\left(\frac{\sigma}{d\left(r_{i}, r_{j}\right)}\right)^{12}-\left(\frac{\sigma}{d\left(r_{i}, r_{j}\right)}\right)^{6}\right],
\end{aligned}
$$

with the diameter $\sigma$ and the well-depth $\epsilon$. The potential is minimal for a particle distance of $d_{m}=\sqrt[6]{2} \sigma \approx 1.2 \sigma$.

Using explicit time stepping the evolution of the particle positions $r_{i}$ can be described by

$$
\begin{aligned}
f_{i}^{n} & =-\frac{\partial}{\partial r_{i}^{n}} U\left(R^{n}\right), \\
a_{i}^{n} & =\frac{f_{i}}{2 m_{i}}, \\
v_{i}^{n+1} & =v_{i}^{n}+a_{i}^{n} \Delta t, \\
r_{i}^{n+1} & =r_{i}^{n}+v_{i}^{n+1} \Delta t,
\end{aligned}
$$

with velocity $v$ and acceleration $a$. As the values of the previous time step for velocity $v_{i}$ and position $r_{i}$ are used, these values need to be saved. If all particle 
interactions are computed the computation will scale with $O\left(N^{2}\right)$, where $N$ is the number of particles. However $v(d) \rightarrow 0$ as $d \rightarrow \infty$ and there exists a cutoff distance $d_{\text {cutoff with }}\|v(d)\|<\delta_{\text {cutoff }}$ for $\delta_{\text {cutoff }}>0$ and all $d>d_{\text {cutoff. }}$ Therefore if the distance between particles is large, the contribution to the force $f_{i}$ is small and can be ignored without changing the simulation result significantly. Therefore by ignoring force contributions smaller than $\delta_{\text {cutoff }}$, particles with a distance $d>d_{\text {cutoff }}$ do not require a calculation of the force contribution. In order to avoid the distance calculation as well, the particles are sorted into cells, so that particles only interact with particles in the same cell and the neighboring cell. That means the edge length of a cubic cell needs to be larger than $d_{\text {cutoff. }}$. This way the amount of calculation reduces to $O(N)$ as the amount of calculations for each particle is limited to $27 N_{\max }$ with the maximum number of particles in a cell $N_{\max }$. Before each time step the particles have to be sorted into their appropriate cell. The time step $\Delta t$ should be small enough, that no particle can move more than one cell.

Each of these cells is associated with a grid point of the overlaying multiphase field load-balancer. A cell belongs to process $p \in P$ if $x \in \Omega_{p}$, i.e. the phase field $\phi_{p}$ is the majority phase field in grid point $x$. In contrast to section 7.2.2 updates are only calculated on process $p$ for cells associate with a grid point $x \in \Omega_{p}$. In order to correctly advance a time step on process $p$, the positions $r_{i}$ and velocities $v_{i}$ of particles in cells, that are neighbors of cells on process $p$, need to be updated. When updating the cell affiliation of the particles, each particle can move up to one cell. Therefore, in order to have the correct particles in the neighboring cells, the neighbors' neighbors need to be updated as well. As each process $p$ has access to the phase field data in the sub-domain $\Omega_{p}$ and its halo, each process knows which process owns a cell, that is up to two cells away from its own cells. This means only cells in the interfaces between phase fields need to be updated and their data communicated. If two phases do not have a common interface, the corresponding processes do not need to interact with each other. As the phase field method tries to minimize the interface energy, the amount of interface points as well as the communication volume is kept low.

The communication is implemented in the following way. Each process selects the cells that are in need of an update and sends a request to the appropriate process, that contains a list of the cells it needs from that process. Then that process will 
pack a buffer containing the particle information for these cells and send it back. That process will unpack the buffer and update the cell affiliations of the particles.

In order to balance the computational load in the system a reasonable choice for the weight $\omega_{p}$ for a process $p$ has to be made. This can be done by time measurement of the computation or based on the number of particles in the sub-domain. In [85] the number of particles on each process is balanced, that means

$$
\omega_{p}^{1}=\sum_{\substack{i \in \Omega_{p} \\ \phi_{p}(i)>0}} n(i),
$$

using the number of atoms $n(i)$ in the cell associated with grid point $i \in \Omega$. As the amount of force computations in a cell scales squarely with the number of particles in that cell, this gives rise to a second heuristic given by

$$
\omega_{p}^{2}=\sum_{\substack{i \in \Omega_{p} \\ \phi_{p}(i)>0}} n(i)^{2},
$$

and as third weight $\omega_{p}^{3}$ the measured wall time for computation on each process is used. As a fourth weight the number of interactions that need to calculated is used

$$
\omega_{p}^{4}=\sum_{\substack{i \in \Omega_{p} \\ \phi_{p}(i)>0}} \sum_{\substack{j \in \Omega_{p} \\\|i-j\|_{\infty} \leq 1}} n(i) n(j) .
$$

\subsubsection{Example: Copper Cube}

In this example a cube of FCC copper is placed slightly of the center of the domain. The total number of cells in each dimension is $60 \times \sqrt[3]{\text { \#Processes }}$ and the edge length of the cube is $8 \times \sqrt[3]{\# \text { Processes }}$ cells. At the beginning of the simulation each cell contains 32 copper atoms of mass $m=63.546 \mathrm{a}$.u.. With a density of $8.96 \mathrm{~g} / \mathrm{cm}^{3}$ the particle density is approximately 0.084 atoms $/ \AA^{3}$. There the edge length of each cell 


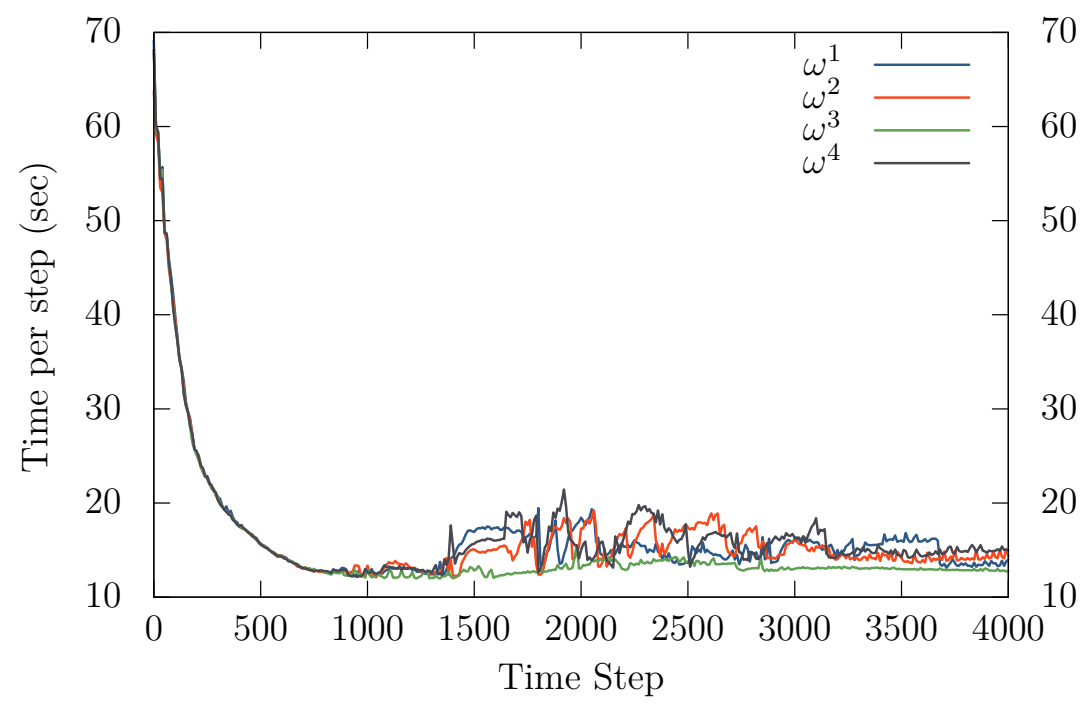

Figure 7.16: Wall time per time step over time using 16 processes and different loadbalancing heuristics.

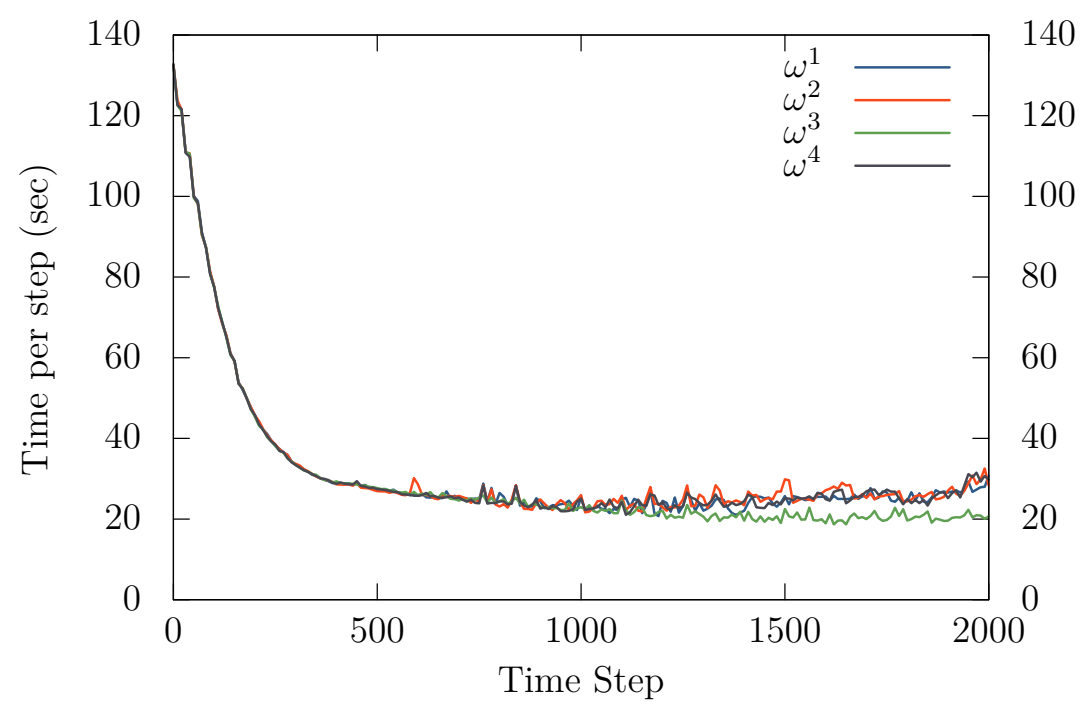

Figure 7.17: Wall time per time step over time using 64 processes and different loadbalancing heuristics. 


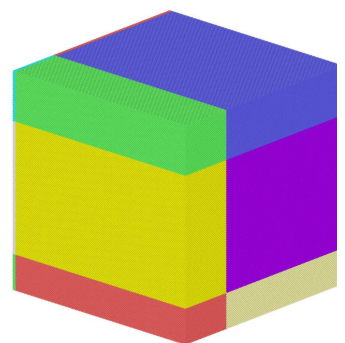

(a) Start configuration

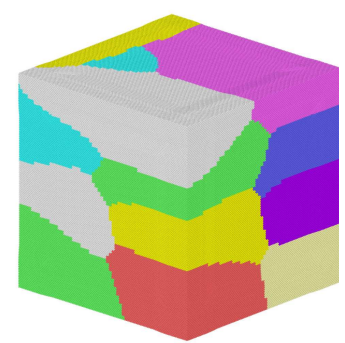

(b) 1000 steps

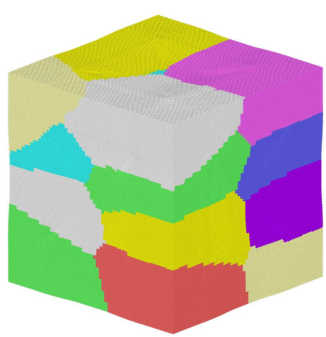

(c) 2000 steps

Figure 7.18: Load-balancing during an MD-Simulation with 16 processes. Color indicates the process rank. Visualization using Ovito [87].

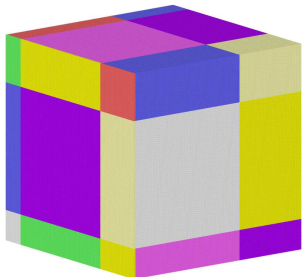

(a) Start configuration

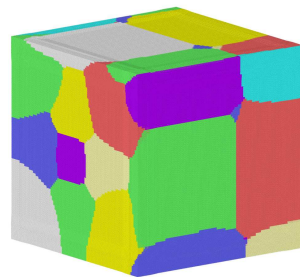

(b) 1000 steps

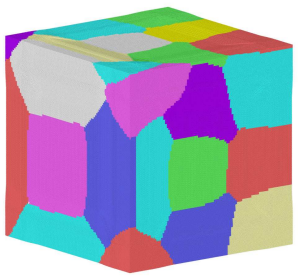

(c) 2000 steps

Figure 7.19: Load-balancing during an MD-Simulation with 64 processes. Color indicates the process rank. Visualization using Ovito [87].

with 32 atoms is $7.25 \AA$. According to [86] the Lennard-Jones potential for copper uses the diameter $\sigma=2.27 \AA$ and the well-depth $\epsilon=0.415 \mathrm{eV} / \mathrm{k}_{\mathrm{b}} \approx 4814 \mathrm{~K}$.

Benchmark results for 16 and 64 processes are shown in figure 7.16 and 7.17 for the different load-balancing heuristics $\omega_{p}^{k}$. For all load-balancing heuristics the wall time significantly improves for the first 250 time steps, after which the reduction in wall time slows down and reaches a plateau after 750 steps. Overall the wall time is reduced by a factor of 4 for the simulation with 16 processes and by a factor of about 6.5 for 64 processes, when using the measured time as the input in the loadbalancer. Using the measured time generally shows the best performance. This is expected because the other weights only approximate the total work and do not take into account effects such as variable clock speeds. Because the cell remain either empty or contain approximately the same number of particles, the three heuristics based on the number of particles give similar results. The domain decompositions are shown in figures 7.18 and 7.19 for weight $\omega^{3}$. 


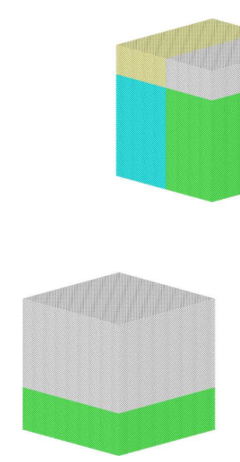

(a) Start configuration

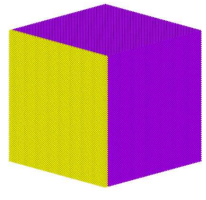

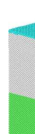

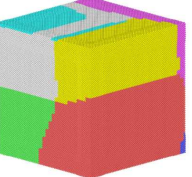

(b) 400 steps
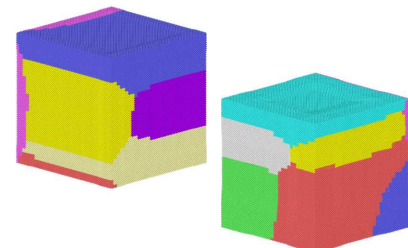

(c) 800 steps

Figure 7.20: Load-balancing during an MD-Simulation with 16 processes. Color indicates the process rank. Visualization using Ovito [87].

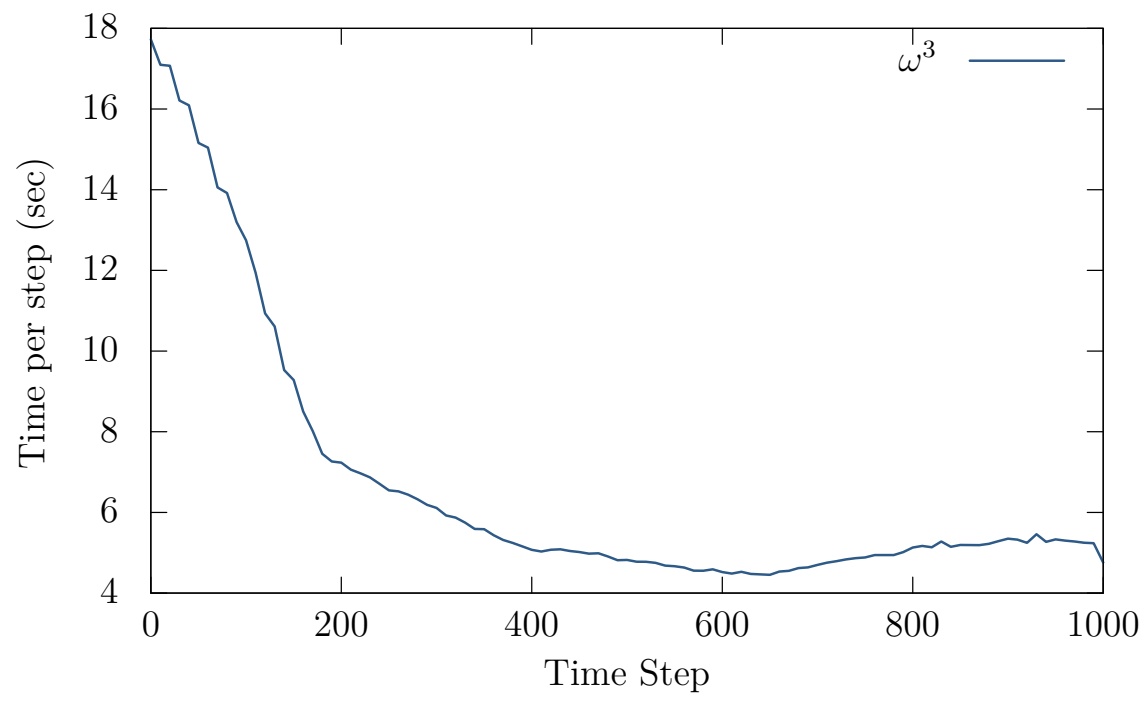

Figure 7.21: Wall time per time step over time using 16 processes for the example with multiple copper cubes in section 7.2.5.

\subsubsection{Example: Multiple Cubes}

This example uses the same basic setup as the copper cube in section 7.2.4, except with three smaller cubes instead of one large cube. The cubes are centered around 
the following coordinates

$$
L\left[\begin{array}{l}
0.7 \\
0.6 \\
0.4
\end{array}\right], L\left[\begin{array}{l}
0.4 \\
0.3 \\
0.3
\end{array}\right], L\left[\begin{array}{l}
0.5 \\
0.4 \\
0.7
\end{array}\right]
$$

with the edge length $L$ of the computational domain. Compared to the copper cube example in section 7.2.4 the cubes have only half the edge length. The start configuration and load-balanced system are shown in figure 7.20. In the load-balanced configuration after 800 steps some processes, such as the one depicted in yellow, contain cells belonging to more than one cube. Figure 7.21 shows the wall time per step over time for the load-balancing informed by the measured time. At first the wall time shows descent for about 190 steps, the descend continues slower until about 650 steps. After which the wall time slightly increases, probably due to the motion of atoms. The load-balancer improves the wall-time by a factor of about three in this simulation. 



\section{Summary and Conclusions}

In this work a parallel implementation of the multiphase field method has been presented in chapter 5 . It allows large scale simulations in three dimensions with a large number of different grains.

In chapter 6 the capabilities of the parallel multiphase field method are shown for applications of grain growth and $\mathrm{Mg}-\mathrm{Al}$ alloy solidification in both actual simulations and derived benchmark problems. Using the advantages of a distributed-memory parallelization simulations of significantly larger problems became possible, that were impossible before due to memory restrictions on single-node architectures. This allowed a simulation of normal grain growth with an initial number of 240000 different phase fields, which reinforce the results obtained from smaller simulations. It is shown that load-balancing can be critical due to the active parameter tracking, which is required for efficient multiphase field simulations. The adaptive splitting and merging of sub-domains has a significant impact on the performance, while the choice of the graph-partitioner only has a minor impact.

Additionally the benchmarks show that this parallelization greatly benefits from a hybrid approach combining MPI and OpenMP, due to the reduction of computational overhead that is caused by the wide halo approach.

In a further study the phase field method was combined with the Lattice-Boltzmann method in order to simulate the effect of solidification on the melt flow through a channel of dendrites. The change in the permeability of a channel during solidification of a $\mathrm{Mg}-\mathrm{Al}$ alloy was investigated using a large scale simulation.

Additionally the multiphase field method was applied to RVE-generation and loadbalancing. A method was presented, that can be used to create sets of Laguerre generator points for given grain size distributions. It has been shown that the multiphase field method can be used to construct Laguerre generator points that result in tessellation with the targeted grain size distribution. It became evident that the 
center of mass points are not accurate enough and additional post-processing is needed. For this the simulated annealing method was used to significantly improve the accuracy of the inverse Laguerre tessellation.

Another application of the multiphase field method was its utilization as a loadbalancing scheme. By associating each process with a different phase field, the driving force can be used to find a domain decomposition that balances the computational load between processes and reduces the amount of communication by minimizing the interface between phase fields.

This approach has been applied to the multiphase field method itself for the simulation of $\mathrm{Mg}-\mathrm{Al}$ alloy solidification and a cell-based particle code.

It was observed that the multiphase field method can be used as a load-balancing scheme. Compared to the parallelization presented in chapter 5 it shows a similar performance for small system sizes. However, the performance suffers when using larger numbers of MPI processes. A significant problem is the overlap of internal grid points. In order to avoid the duplicated calculations on internal grid points, significant changes to the implementation of OpenPhase would be required.

It was shown that the multiphase field load-balancer can also be applied to cellbases particle methods. This method could be very interesting for dynamic systems in which the computational load moves over periodic boundaries as phase fields can also use periodic boundary conditions.

It can be seen that the multiphase field method is a powerful tool for a wide variety of applications, which include but are not limited to materials simulations. 


\section{Appendix}

\subsection{Parallel OpenPhase Tutorial}

The following will give a quick overview on how to utilize the MPI-parallelization of OpenPhase.

\subsubsection{Installation}

The MPI-version of OpenPhase presented in the work and in [3] can be obtained by checking of:

svn co http://openphase-svn.icams.rub.de/svn/OpenPhase/branches/Marvin/ OpenPhase_CPiP

The first step then is the compilation of the library in the parallel mode by make SETTINGS="parallel"

Other settings such as debug, silent and vulcan can be added. The settings vulcan enables optimization for the parallel cluster Vulcan at ICAMS.

\subsubsection{Getting Started}

As an example we will convert the Hexagonal Dendrite example, see examples/HexDendr/HexDendr.cpp in your OpenPhase folder, to distributed memory pa- 
rallelism. We create a new folder called HexDendrParallel inside the examples folder and and copy the folder ProjectInput and the Makefile from HexDendr to HexDendrParallel. Then we create a file named HexDendrParallel.cpp in that folder HexDendrParallel. Each parallel program in OpenPhase begins with the definition of the halo size by defining BOUNDARYSIZE. In our case we select nine boundary cells. Next we include Parallelism.h and mpi.h. We select the namespace openphase and afterwards we create to classes, the first named HexDendrBlock describes a block as presented in section 5. It is derived from ParallelBlock which takes template arguments for the type of interface energy, mobility as well as specialized classes of phase field, temperature and composition. We give this class two functions Init and DoTimestep. We call the other class HexDendrParallel, the class is derived from Parallelism and takes a BlockFormat as template parameter. This class gets three functions Run, InitialConditions and synchronize. Finally we add the main function to our code, that initializes MPI and sets MPI_SIZE and MPI_RANK. It also should call MPI_Finalize before exiting the program.

Going further we will expand the main function. The values OPP_BlockSize_* and OPP_BlockNumber_* are used to set the size and number of blocks in each dimension. After that we create an object PS of type HexDendrParallel with the template argument HexDendrBlock, that we defined above. After that we initialize the object and set in this order the grade of verbosity, periodicity in $\mathrm{X}, \mathrm{Y}$ and $\mathrm{Z}$ direction, the parameter $\Theta$ as defined in section 5 and the initial load balancing tolerance. After that we executed the Run function of PS.

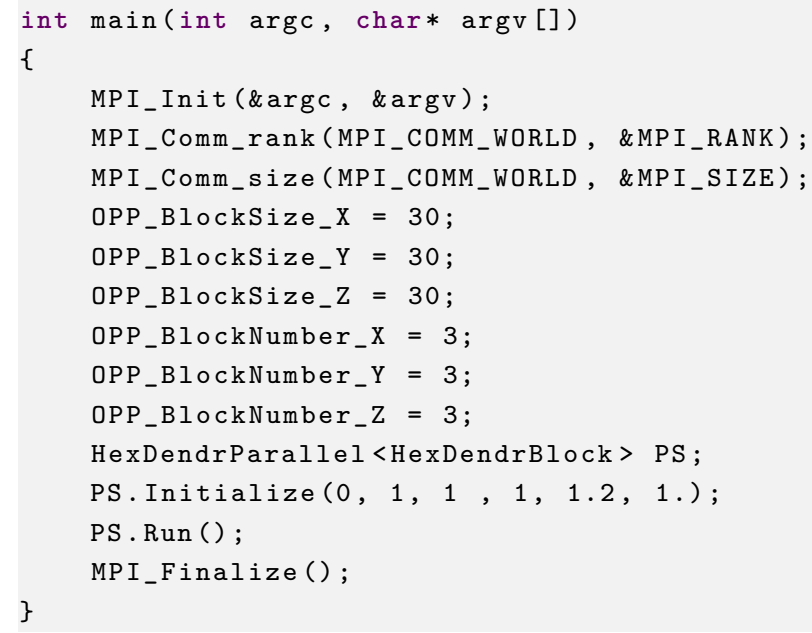




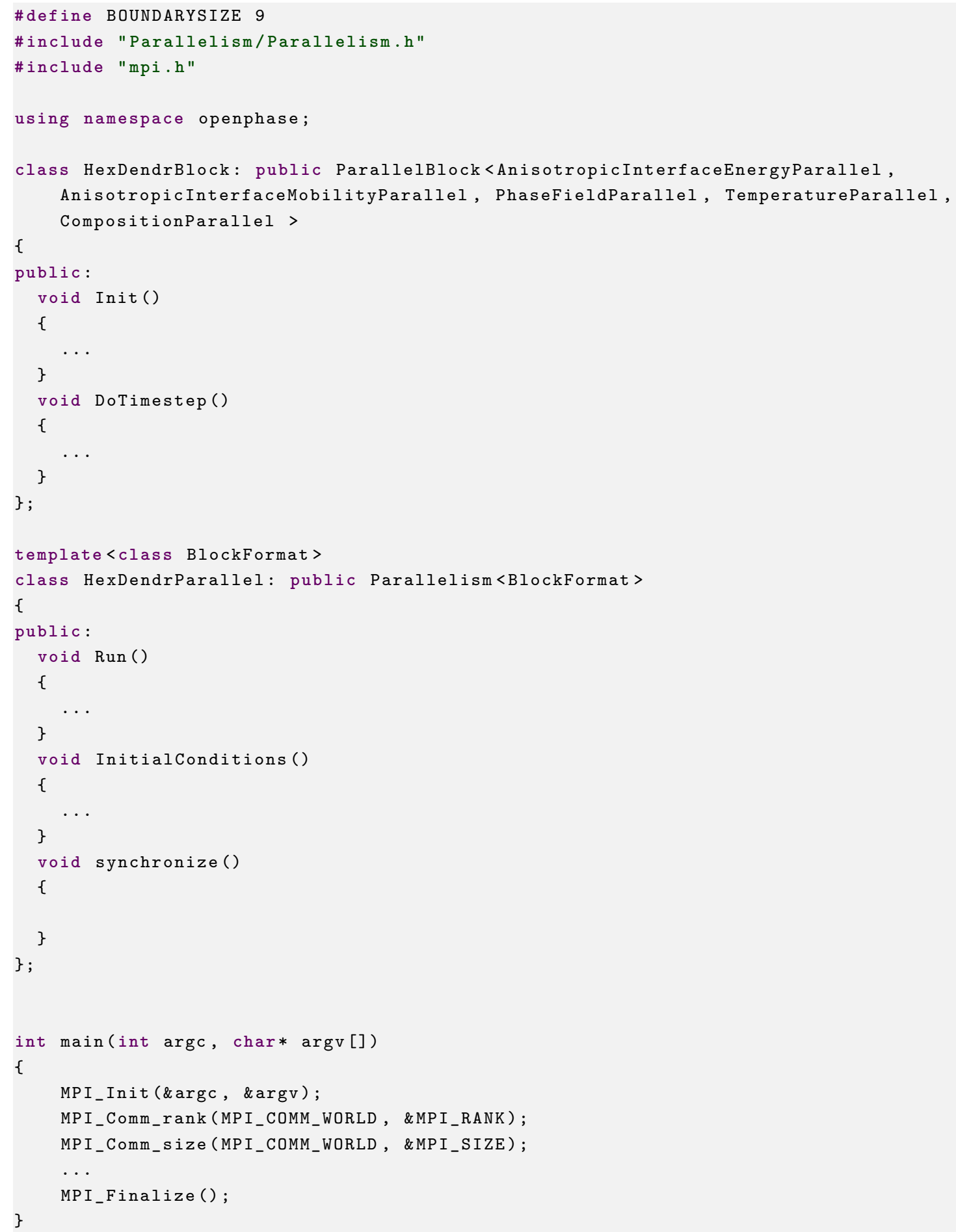

Code 1: This is how your HexDendrParallel.cpp should look like. 
After that we look at the Run function itself, which in the first step sets the InitialConditions. Then it starts a time loop in which it iterates of the blocks on this process and computes a time step for each of them. After all blocks have completed their calculation the synchronize function is called which implements the halo exchange. Finally we globally advance the time step by calling timestep.

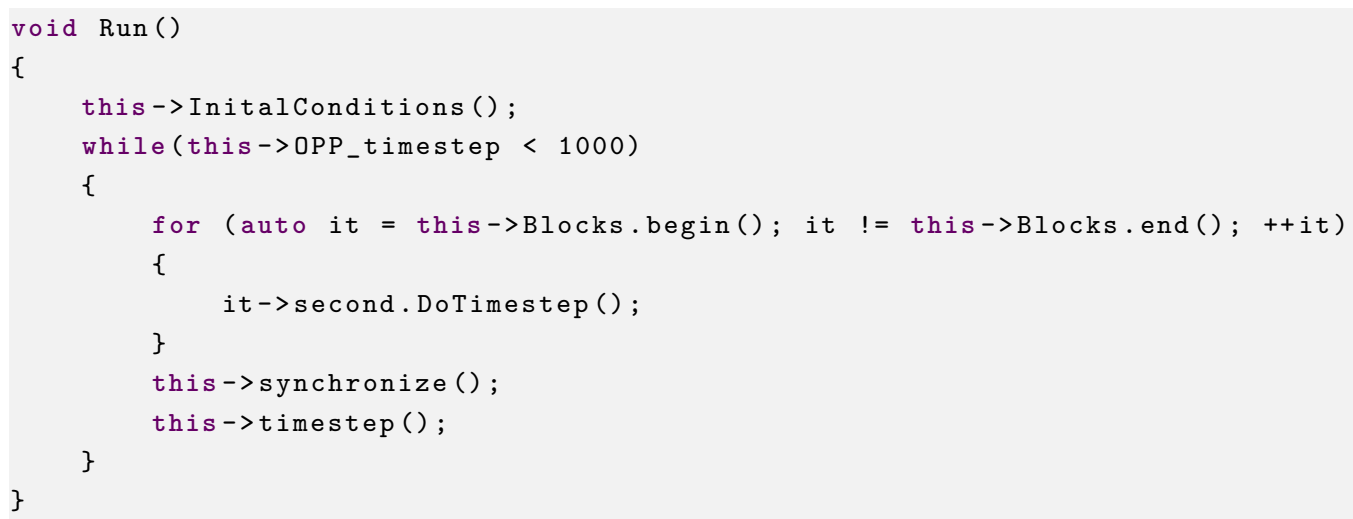

The synchronize function is simple and exchanges the principal values with the neighboring blocks, which in this case is phase field, composition and temperature, which have already predefined functions.

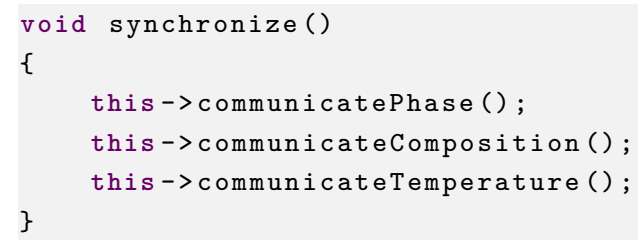

Finally we add InitialConditions in which we plant a sphere of phase index 1 submerged in fluid of phase index 1. Afterward we set both the initial composition and temperature and synchronized the data before the first time step.

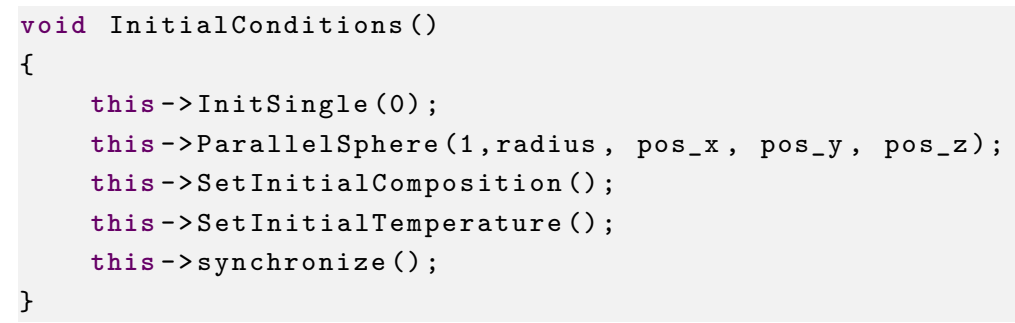

Finally we need to implement the Init and DoTimestep functions of the HexDendrBlock. Init essentially looks like the firs few lines of any OpenPhase program 


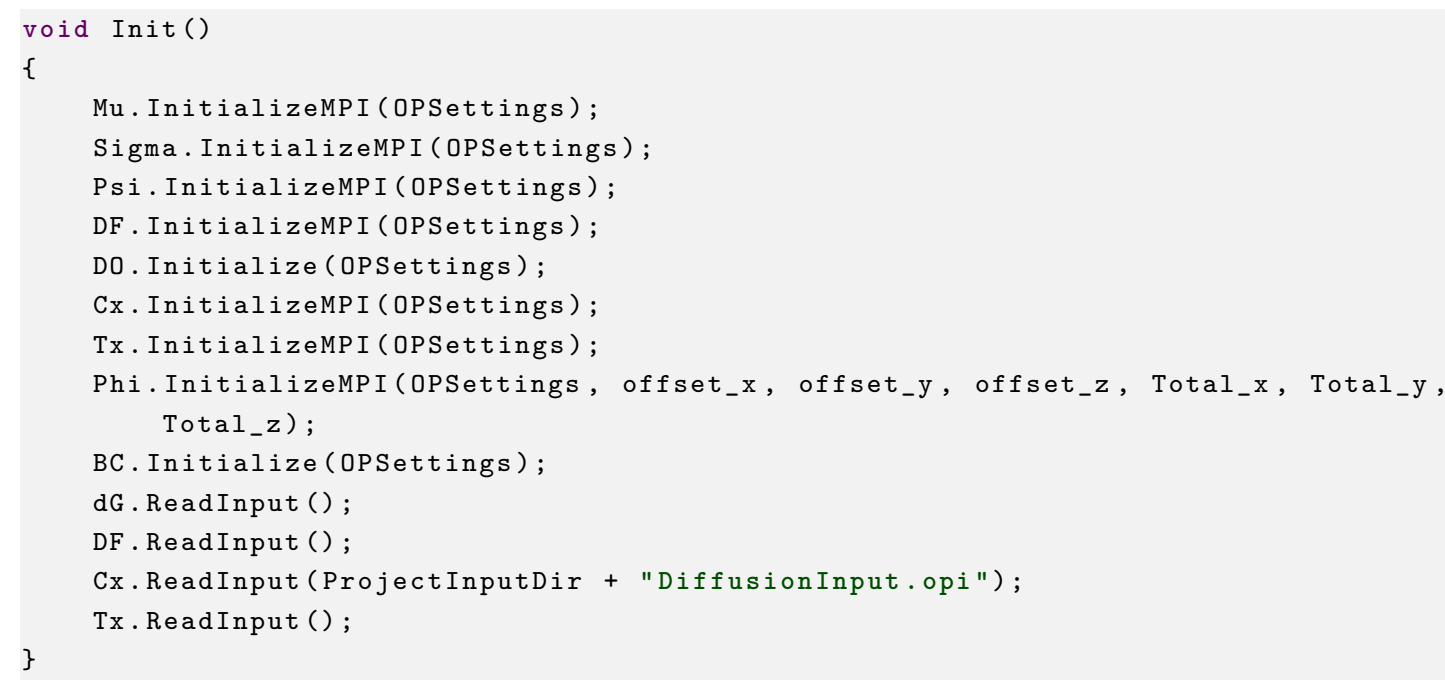

and DoTimestep looks like the inside of the time loop in a serial OpenPhase program.

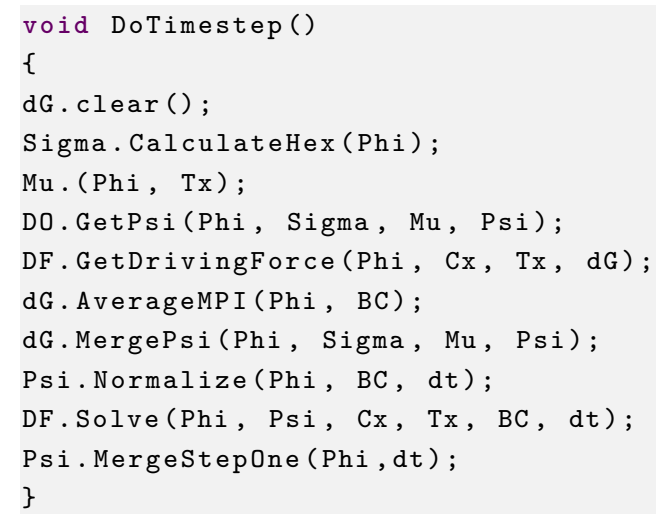

Now you can compile the program by using again in the HexDendrParallel folder

make SETTINGS = "parallel "

Now you should be able to execute the program using mpirun -np 2 ./HexDendrParallel 



\section{Bibliography}

[1] M. Tegeler, A. Monas, and G. Sutmann, "Massively parallel multiphase field simulations," in Proceedings of Fourth International Conference on Parallel, Distributed, Grid and Cloud Computing for Engineering, 2015. 1, 5, 5.2, 5.2.1, $5.2 .2,6.3,6.5 .3$

[2] M. Tegeler, A. Monas, O. Shchyglo, I. Steinbach, and F. Varnik, "Effect of microstructure during dendritic solidification on melt flow: A phase-field-latticeboltzmann study," in Proceedings of the 5th Decennial International Conference on Solidification Processing, 2017. 1, 6.6

[3] M. Tegeler, O. Shchyglo, R. D. Kamachali, A. Monas, I. Steinbach, and G. Sutmann, "Parallel multiphase field simulations with OpenPhase," Computer Physics Communications, vol. 215, pp. -, 2017. 1, 2.1, 5, 6, 9.1.1

[4] A. Schölmerich, "Promotionsordnung der Fakultät für Maschinenbau der RuhrUniversität Bochum vom 21. September 2016," Amtliche Bekanntmachungen der Ruhr-Universität Bochum, 2016. (document)

[5] L.-Q. Chen, "Phase-field models for microstructure evolution," Annual Review of Materials Research, vol. 32, no. 1, pp. 113-140, 2002. 1, 6.1

[6] I. Steinbach, "Phase-field models in materials science," Modelling and Simulation in Materials Science and Engineering, vol. 17, no. 7, p. 073001, 2009. 1, $2,2.1,2.2,6.1,6.4,6.4$

[7] I. Steinbach, "Why solidification? why phase-field?," JOM, vol. 65, no. 9, pp. 1096-1102, 2013. 1 
[8] I. Häusler, C. Schwarze, M. U. Bilal, D. V. Ramirez, W. Hetaba, R. D. Kamachali, and B. Skrotzki, "Precipitation of $\mathrm{t} 1$ and $\theta \prime$ phase in al4Cu1li0.25mn during age hardening: Microstructural investigation and phasefield simulation," Materials, vol. 10, 2017. 1

[9] R. D. Kamachali, E. Borukhovich, N. Hatcher, and I. Steinbach, "DFTsupported phasefield study on the effect of mechanically driven fluxes in ni4ti3 precipitation," Modelling and Simulation in Materials Science and Engineering, vol. 22, 2014. 1

[10] C. Schwarze, R. D. Kamachali, and I. Steinbach, "Phasefield study of zener drag and pinning of cylindrical particles in polycrystalline materials," Acta Materialia, vol. 106, 2016. 1

[11] R. D. Kamachali, A. Abbondandolo, K. Siburg, and I. Steinbach, "Geometrical grounds of mean field solutions for normal grain growth," Acta Materialia, vol. $90,2015.1,6.1$

[12] R. D. Kamachali and I. Steinbach, "3D phasefield simulation of grain growth: topological analysis versus meanfield approximations," Acta Materialia, vol. 60, 2012. 1, 6.1, 6.1.1

[13] R. D. Kamachali, J. Hua, I. Steinbach, and A. Hartmaier, "Multiscale simulations on the grain growth process in nanostructured materials," International Journal of Materials Research, vol. 11, 2010. 1

[14] A. Monas, P. Bloembergen, W. Dong, O. Shchyglo, and I. Steinbach, "Simulations of the eutectic transformations in the platinum-carbon system," International Journal of Thermophysics, vol. 36, 2015. 1

[15] E. Borukhovich, M. Boeff, A. Monas, M. Tegeler, S. Kim, C. Oh, and I. Steinbach, "Fullfield simulation of solidification and forming of polycrystals," in MATEC Web of Conferences, vol. 80, p. 02014, 01 2016. 1

[16] R. D. Kamachali, S.-J. Kim, and I. Steinbach, "Texture evolution in deformed az31 magnesium sheets: Experiments and phase-field study," Computational Materials Science, vol. 104, pp. 193 - 199, 2015. 1

[17] "Openphase." www .openphase.de. 1 
[18] W. L. George and J. A. Warren, "A parallel 3D dendritic growth simulator using the phase-field method," Journal of Computational Physics, vol. 177, no. 2, pp. 264-283, 2002. 1, 4.4

[19] A. Vondrous, M. Selzer, J. Hötzer, and B. Nestler, "Parallel computing for phase-field models," Int. J. High Perform. Comput. Appl., vol. 28, pp. 61-72, Feb. 2014. 1, 4.4

[20] T. Shimokawabe, T. Aoki, T. Takaki, T. Endo, A. Yamanaka, N. Maruyama, A. Nukada, and S. Matsuoka, "Peta-scale phase-field simulation for dendritic solidification on the tsubame 2.0 Supercomputer," in Proceedings of 2011 International Conference for High Performance Computing, Networking, Storage and Analysis, SC '11, (New York, NY, USA), pp. 3:1-3:11, ACM, 2011. 1, 4.4

[21] "Pace3d." https://www.hs-karlsruhe.de/cmse/pace-3d-software/. 1, 4.4

[22] "Micress @." http://web.micress.de/. 1, 4.4

[23] I. Steinbach, "Phase-field model for microstructure evolution at the mesoscopic scale," Annual Review of Materials Research, vol. 43, no. 1, pp. 89-107, 2013. $2,2.1$

[24] Y. Li and J. Kim, "Multiphase image segmentation using a phase-field model," Computers \&S Mathematics with Applications, vol. 62, no. 2, pp. 737 - 745, 2011. 2

[25] M. Beněs, V. Chalupecký, and K. Mikula, "Geometrical image segmentation by the allen-cahn equation," Applied Numerical Mathematics, vol. 51, no. 2, pp. $187-205,2004.2$

[26] S. Vedantam and B. S. V. Patnaik, "Efficient numerical algorithm for multiphase field simulations," Phys. Rev. E, vol. 73, p. 016703, Jan. 2006. 2.2

[27] Y. H. Qian, D. D'Humières, and P. Lallemand, "Lattice bgk models for navierstokes equation," EPL (Europhysics Letters), vol. 17, no. 6, p. 479, 1992. 3

[28] S. Chen and G. D. Doolen, "Lattice boltzmann method for fluid flows," Annual Review of Fluid Mechanics, vol. 30, no. 1, pp. 329-364, 1998. 3, 3 
[29] F. Varnik, M. Gross, N. Moradi, G. Zikos, P. Uhlmann, P. Müller-Buschbaum, D. Magerl, D. Raabe, I. Steinbach, and M. Stamm, "Stability and dynamics of droplets on patterned substrates: insights from experiments and lattice boltzmann simulations," Journal of Physics: Condensed Matter, vol. 23, no. 18, p. $184112,2011.3,3$

[30] D. A. Wolf-Gladrow, Lattice-gas cellular automata and lattice Boltzmann models: an introduction. Springer, 2004. 3

[31] T. Krüger, F. Varnik, and D. Raabe, "Second-order convergence of the deviatoric stress tensor in the standard bhatnagar-gross-krook lattice boltzmann method," Phys. Rev. E, vol. 82, p. 025701, Aug. 2010. 3.1

[32] X. Shan and H. Chen, "Lattice boltzmann model for simulating flows with multiple phases and components," Phys. Rev. E, vol. 47, pp. 1815-1819, Mar. 1993. 3.2

[33] X. Shan and H. Chen, "Simulation of nonideal gases and liquid-gas phase transitions by the lattice boltzmann equation," Phys. Rev. E, vol. 49, pp. 2941-2948, Apr. 1994. 3.2

[34] A. Kupershtokh, D. Medvedev, and D. Karpov, "On equations of state in a lattice boltzmann method," Computers $\& 3$ Mathematics with Applications, vol. 58, no. 5, pp. 965 - 974, 2009. Mesoscopic Methods in Engineering and Science. 3.2

[35] C. K. Aidun and Y. Lu, "Lattice boltzmann simulation of solid particles suspended in fluid," Journal of Statistical Physics, vol. 81, no. 1, pp. 49-61, 1995. 3.3

[36] H. Sutter, "The free lunch is over a fundamental turn toward concurrency in software," Dr. Dobb's Journal, 2005. 4

[37] M. McCool, J. Reinders, and A. Robison, Structured Parallel Programming: Patterns for Efficient Computation. San Francisco, CA, USA: Morgan Kaufmann Publishers Inc., 1st ed., 2012. 4, 4.1.2 
[38] L. Dagum and R. Menon, "Openmp: an industry standard API for sharedmemory programming," Computational Science \& Engineering, IEEE, vol. 5, no. 1 , pp. $46-55,1998.4 .1 .2$

[39] G. M. Amdahl, "Validity of the single processor approach to achieving large scale computing capabilities," in Proceedings of the April 18-20, 1967, Spring Joint Computer Conference, AFIPS '67 (Spring), (New York, NY, USA), pp. 483-485, ACM, 1967. 4.2

[40] J. L. Gustafson, Gustafsons Law, pp. 819-825. Boston, MA: Springer US, 2011. 4.2

[41] J. Rosam, A Fully implicit, Fully adaptive multigrid method for multiscale phase-field modelling. PhD thesis, University of Leeds, 2007. 4.4

[42] J. Hötzer, Massiv-parallele und großskalige Phasenfeldsimulationen zur Untersuchung der Mikrostrukturentwicklung. PhD thesis, Karlsruher Institut für Technologie, 2017. 4.4

[43] G. Karypis and V. Kumar, "Multilevel algorithms for multi-constraint graph partitioning," in Proceedings of the 1998 ACM IEEE Conference on Supercomputing, SC 98, (Washington, DC, USA), pp. 1-13, IEEE Computer Society, 1998. 5

[44] C. Chevalier and F. Pellegrini, "Pt-scotch: A tool for efficient parallel graph ordering," Parallel Computing, vol. 34, no. 6-8, pp. 318-331, 2008. Parallel Matrix Algorithms and Applications. 5

[45] F. B. Kjolstad and M. Snir, "Ghost cell pattern," in Proceedings of the 2010 Workshop on Parallel Programming Patterns, ParaPLoP '10, (New York, NY, USA), pp. 4:1-4:9, ACM, 2010. 5, 5.1.1

[46] K. Andreev and H. Räcke, "Balanced graph partitioning," in Proceedings of the Sixteenth Annual ACM Symposium on Parallelism in Algorithms and Architectures, SPAA '04, (New York, NY, USA), pp. 120-124, ACM, 2004. 5.2.1 
[47] E. G. Boman, U. V. Catalyurek, C. Chevalier, and K. D. Devine, "The Zoltan and Isorropia parallel toolkits for combinatorial scientific computing: Partitioning, ordering, and coloring," Scientific Programming, vol. 20, no. 2, pp. 129150, 2012. 5.2.1

[48] G. Karypis and V. Kumar, "A fast and high quality multilevel scheme for partitioning irregular graphs," SIAM Journal on Scientific Computing, vol. 20, no. 1, pp. 359-392, 1998. 5.2.1

[49] D. E. King, "Dlib-ml: A machine learning toolkit," Journal of Machine Learning Research, vol. 10, pp. 1755-1758, 2009. 5.2.3

[50] B. Welch, M. Unangst, Z. Abbasi, G. Gibson, B. Mueller, J. Small, J. Zelenka, and B. Zhou, "Scalable performance of the panasas parallel file system," in Proceedings of the 6th USENIX Conference on File and Storage Technologies, FAST'08, (Berkeley, CA, USA), pp. 2:1-2:17, USENIX Association, 2008. 5.3

[51] F. Schmuck and R. Haskin, "Gpfs: A shared-disk file system for large computing clusters," in Proceedings of the 1st USENIX Conference on File and Storage Technologies, FAST '02, (Berkeley, CA, USA), USENIX Association, 2002. 5.3

[52] P. Wautelet, "Best practices for parallel io and mpi-io hints." PATC Training session Parallel filesystems and parallel IO librarie, 2015. 5.3

[53] Kitware, The VTK User's Guide 11th Edition. Kitware, Inc, 2010. 5.3

[54] M. T. Lusk, "A phase-field paradigm for grain growth and recrystallization," Proceedings of the Royal Society of London A: Mathematical, Physical and Engineering Sciences, vol. 455, no. 1982, pp. 677-700, 1999. 6.1

[55] L.-Q. Chen, "A novel computer simulation technique for modeling grain growth," Scripta Metallurgica et Materialia, vol. 32, no. 1, pp. 115-120, 1995. 6.1

[56] D. Fan and L.-Q. Chen, "Computer simulation of grain growth using a continuum field model," Acta Materialia, vol. 45, no. 2, pp. 611-622, 1997. 6.1

[57] C. Krill III and L.-Q. Chen, "Computer simulation of 3-D grain growth using a phase-field model," Acta Materialia, vol. 50, no. 12, pp. 3059-3075, 2002. 6.1 
[58] Y. Suwa, Y. Saito, and H. Onodera, "Phase field simulation of grain growth in three dimensional system containing finely dispersed second-phase particles," Scripta Materialia, vol. 55, no. 4, pp. 407-410, 2006. 6.1

[59] C. H. Rycroft, "Voro++: A three-dimensional voronoi cell library in c++," Chaos, vol. 19, no. 4, pp. -, 2009. 6.1

[60] J. Ahrens, B. Geveci, and C. Law, ParaView: An End-User Tool for Large Data Visualization. Elsevier, 2005. 6.1

[61] U. Ayachit, The ParaView Guide: A Parallel Visualization Application. Kitware, 2015. 6.1

[62] H. Childs, E. S. Brugger, K. S. Bonnell, J. S. Meredith, M. Miller, B. J. Whitlock, and N. Max, "A contract-based system for large data visualization," in Proceedings of IEEE Visualization 2005, pp. 190-198, 2005. 6.1

[63] R. D. Kamachali, Grain boundary motion in polycrystalline materials. PhD thesis, RuhrUniversity Bochum, Interdisciplinary Centre for Advanced Materials Simulation, STKS, 2013. 6.1.1

[64] M. Hillert, "On the theory of normal and abnormal grain growth," Acta Metallurgica, vol. 13, no. 3, pp. 227-238, 1965. 6.1.1, 6.4

[65] M.-C. Zhao, M. Liu, G. Song, and A. Atrens, "Influence of the beta-phase morphology on the corrosion of the mg alloy az91," Corrosion Science, vol. 50, no. 7 , pp. 1939-1953, 2008. 6.3

[66] A. Monas, O. Shchyglo, S.-J. Kim, C. D. Yim, D. Höche, and I. Steinbach, "Divorced eutectic solidification of mg-al alloys," JOM, vol. 67, no. 8, pp. 18051811, 2015. 6.3, 6.6

[67] A. Monas, O. Shchyglo, D. Höche, M. Tegeler, and I. Steinbach, "Dual-scale phase-field simulation of mg-al alloy solidification," IOP Conference Series: Materials Science and Engineering, vol. 84, no. 1, p. 012069, 2015. 6.3, 6.6

[68] I. Steinbach and M. Apel, "Multi phase field model for solid state transformation with elastic strain," Physica D, vol. 217, 2006. 6.4 
[69] M. Muskat and M. W. Meres, "The flow of heterogeneous fluids through porous media," Physics, vol. 7, no. 9, pp. 346-363, 1936. 6.6.2

[70] F. Varnik, D. Dorner, and D. Raabe, "Roughnessinduced flow instability : A lattice boltzmann study," Journal of Fluid Mechanics, vol. 573, 2007. 6.6.2

[71] P. J. McGonigal, A. D. Kirshenbaum, and A. V. Grosse, "The liquid temperature range, density, and critical constants of magnesium," vol. 66, pp. 737-740, 04 1962. 6.6 .2

[72] J. Eiken, I. Steinbach, G. Klaus, and A. Bührig-Polaczek, "Grain refinement and grain morphology evolution in magnesium castings investigated by phasefield simulations.," Magnesium Technology, 2008. 6.6.3

[73] W. Müller, Mechanische Grundoperationen und ihre Gesetzmäßigkeiten. Oldenbourg, 2008. 6.6.3

[74] I. Vecchio, K. Schladitz, and C. Redenbach, "Laguerre tessellations: fitting a model to rigid closed-cell polymer foams," in Proceedings Cellular Materials, (Dresden), Deutsche Gesellschaft für Materialkunde, 2012. 7

[75] M. Boeff, Micromechanical modelling of fatigue crack initiation and growth. PhD thesis, Ruhr-Universität Bochum, 2016. 7, 7.1

[76] C. Redenbach, I. Shklyar, and H. Andr, "Laguerre tessellations for elastic stiffness simulations of closed foams with strongly varying cell sizes," International Journal of Engineering Science, vol. 50, no. 1, pp. 70 - 78, 2012. 7, 7.1

[77] Q. Duan, D. P. Kroese, T. Brereton, A. Spettl, and V. Schmidt, "Inverting laguerre tessellations," The Computer Journal, vol. 57, no. 9, p. 1431, 2014. 7.1 .2

[78] A. Liebscher, D. Jeulin, and C. Lantuéjoul, "Stereological reconstruction of polycrystalline materials," Journal of Microscopy, vol. 258, no. 3, pp. 190-199, 2015. 7.1 .2

[79] A. Spettl, T. Brereton, Q. Duan, T. Werz, C. E. K. III, D. P. Kroese, and V. Schmidt, "Fitting laguerre tessellation approximations to tomographic image data," Philosophical Magazine, vol. 96, no. 2, pp. 166-189, 2016. 7.1.2 
[80] S. Kirkpatrick, C. D. Gelatt, and M. P. Vecchi, "Optimization by simulated annealing," Science, vol. 220, no. 4598, pp. 671-680, 1983. 7.1.2

[81] P. J. Davis, "Leonhard euler's integral: A historical profile of the gamma function: In memoriam: Milton abramowitz," The American Mathematical Monthly, vol. 66, no. 10, pp. 849-869, 1959. 7.1.2

[82] R. Quey, P. Dawson, and F. Barbe, "Large-scale 3d random polycrystals for the finite element method: Generation, meshing and remeshing," Computer Methods in Applied Mechanics and Engineering, vol. 200, no. 17, pp. 1729 1745, 2011. 7.1.5

[83] M. P. Allen et al., "Introduction to molecular dynamics simulation," Computational soft matter: from synthetic polymers to proteins, vol. 23, pp. 1-28, 2004. $7.2,7.2 .3$

[84] J. J. Monaghan, "Smoothed particle hydrodynamics," Annual Review of Astronomy and Astrophysics, vol. 30, no. 1, pp. 543-574, 1992. 7.2

[85] C. Begau and G. Sutmann, "Adaptive dynamic load-balancing with irregular domain decomposition for particle simulations," Computer Physics Communications, vol. 190 , pp. $51-61,2015$. 7.2 .3

[86] P. M. Agrawal, B. M. Rice, and D. L. Thompson, "Predicting trends in rate parameters for self-diffusion on fcc metal surfaces," Surface Science, vol. 515, no. 1 , pp. $21-35,2002.7 .2 .4$

[87] A. Stukowski, "Visualization and analysis of atomistic simulation data with ovito-the open visualization tool," Modelling and Simulation in Materials Science and Engineering, vol. 18, no. 1, p. 015012, 2010. 7.18, 7.19, 7.20 


\section{Marvin Tegeler}

\section{Curriculum Vitae of Marvin Tegeler}

\section{About Me}

Birth $07 / 31 / 1985$ in Gelsenkirchen

Nationality German

\section{Education}

Doctoral Studies in Mechanical Engineering

since 2/2013 Ruhr-Universität Bochum

Interdisciplinary Centre For Advanced Materials Simulation

Department High-Performance Computing in Materials Science

Diploma in Mathematics

4/2006 - 8/2012 Ruprecht-Karls-Universität Heidelberg, grade: 1.0

Diploma Thesis Numerische Simulation der Sternentstehung

\section{Civilian Service}

7/2005 - 3/2006 Glück-Auf-Werkstatt for people with mental handicaps in Herten

\section{School Education}

7/1996 - 6/2005 Städtisches Gymnasium Herten, grade: 2.2

7/1992 - 6/1996 Barbaraschule in Herten

\section{Publications}

Marvin Tegeler, Alexander Monas, Oleg Shchyglo, Ingo Steinbach, Fathollah Varnik, Effect of Microstructure during Dendritic Solidification on Melt Flow: A Phase-field-lattice-Boltzmann Study, Proceedings of the 6th Decennial International Conference on Solidification Processing, July 2017

Marvin Tegeler, Oleg Shchyglo, Reza Darvishi Kamachali, Alexander Monas, Ingo Steinbach, Godehard Sutmann, Parallel multiphase field simulations with OpenPhase, Computer Physics Communications, June 2017

Efim Borukhovich, Martin Boeff, Alexander Monas, Marvin Tegeler, Se-Jong Kim, Chang-Seok Oh, Ingo Steinbach, Full-field simulation of solidification and forming of polycrystals, MATEC Web Conf., 2016

Alexander Monas, Oleg Shchyglo, Daniel Höche, Marvin Tegeler, Ingo Steinbach, Dual-scale phase-field simulation of $\mathrm{Mg}$-Al alloy solidification, IOP Conference Series Materials Science and Engineering, June 2015

Marvin Tegeler, Alexander Monas, Godehard Sutmann, Massively parallel multiphase field simulations, Proceedings of the 4th International Conference on Parallel, Distributed, Grid and Cloud Computing for Engineering, March 2015

Bochum, October 12, 2017

$$
\text { Marvin Tegla }
$$

